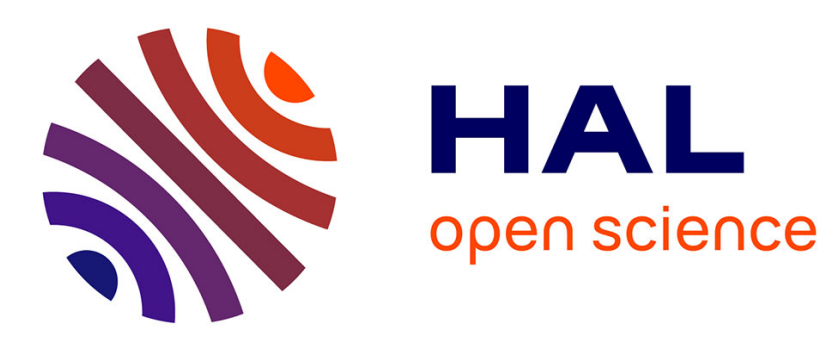

\title{
The Discrete Duality Finite Volume method for the Stokes equations on 3-D polyhedral meshes
}

\author{
Stella Krell, Gianmarco Manzini
}

\section{To cite this version:}

Stella Krell, Gianmarco Manzini. The Discrete Duality Finite Volume method for the Stokes equations on 3-D polyhedral meshes. SIAM Journal on Numerical Analysis, 2012, 50 (2), pp.808-837. $10.1137 / 110831593$. hal-00448465v2

\section{HAL Id: hal-00448465 \\ https://hal.science/hal-00448465v2}

Submitted on 14 Jun 2011

HAL is a multi-disciplinary open access archive for the deposit and dissemination of scientific research documents, whether they are published or not. The documents may come from teaching and research institutions in France or abroad, or from public or private research centers.
L'archive ouverte pluridisciplinaire HAL, est destinée au dépôt et à la diffusion de documents scientifiques de niveau recherche, publiés ou non, émanant des établissements d'enseignement et de recherche français ou étrangers, des laboratoires publics ou privés. 


\title{
The Discrete Duality Finite Volume method for the Stokes equations on 3-D polyhedral meshes
}

\author{
Stella Krell * $\quad$ Gianmarco Manzini ${ }^{\dagger \ddagger}$
}

\begin{abstract}
We develop a Discrete Duality Finite Volume (DDFV) method for the three-dimensional steady Stokes problem with a variable viscosity coefficient on polyhedral meshes. Under very general assumptions on the mesh, which may admit non-convex and non-conforming polyhedrons, we prove the stability and well-posedness of the scheme. We also prove the convergence of the numerical approximation to the velocity, velocity gradient and pressure, and derive a priori estimates for the corresponding approximation error. Final numerical experiments confirm the theoretical predictions.
\end{abstract}

Keywords: Discrete duality finite volume method, 3-D Stokes equations, variable viscosity.

\section{Introduction}

The numerical approximation of the steady Stokes problem with variable viscosity requires the discretization of the symmetric gradient of a divergence-free velocity field. To address this issue in the framework of the Finite Volume method, the full gradient of the vector variable must be discretized at each control volume interface, which may require to set unknowns at mesh vertices. A number of techniques have been proposed in the Finite Volume literature of the last decade to approximate the gradient of a scalar field $[2,10,20,25,28,24,31,32,34,35]$. A comparison of the performance of some of these schemes for three-dimensional (3-D) diffusion problems with anisotropic permeabilities is found in the benchmark of the FVCA-6 Conference [29] held in Prague, in 2011.

The DDFV method was originally developed to approximate the solution of the Poisson equation on a large class of 2-D polygonal meshes, which may include non-conformal and distorted control volumes $[24,31]$. The DDFV formulation in two spatial dimensions is based on two Finite Volume schemes, and consists of a system of flux balance equations for the cells of the primal mesh and for the cells that are built around the vertices of the primal cells, i.e., the dual mesh. These flux balance equations make use of a numerical flux that is based on an approximate gradient formula defined for the diamond cells, the control volumes of a third mesh superimposed to the primal and the dual mesh.

The DDFV method has been successfully employed in the numerical approximation of the linear diffusion equation with anisotropic permeabilities [13, 24, 31], the steady convection-diffusion equation [21], the div-curl problem [23] of electrostatics and magnetostatics, the non-linear elliptic equation involving Leray-Lions operators $[1,12]$, the bidomain equation modeling the electromagnetic activity of the heart [19]. Regarding the numerical approximation to the Stokes problem, two different twodimensional DDFV formulations have been proposed in the literature: the first one is discussed in the doctoral dissertation of Reference [22], the second one is found in [33]. In the first approach, the

\footnotetext{
*Université de Provence, Laboratoire d'Analyse, Topologie et Probabilités, 39 rue F. Joliot-Curie, 13453 Marseille Cedex 13, France e-mail: krell@latp.univ-mrs.fr

${ }^{\dagger}$ Istituto di Matematica Applicata e Tecnologie Informatiche (IMATI) - CNR, via Ferrata 1, I - 27100 Pavia, Italy e-mail: Marco.Manzini@imati.cnr.it Italy

¥Centro di Simulazione Numerica Avanzata (CeSNA) - IUSS Pavia, v.le Lungo Ticino Sforza 56, I - 27100 Pavia,
} 
pressure degrees of freedom are defined at mesh vertices and cell centers, while the velocity is approximated by a piecewise constant vector in the diamond cells. The resulting scheme is well-posed, but only constant viscosity fields are easily treatable. This fact motivated the development of the second approach, in which the velocity degrees of freedom are attached to cell centers and mesh vertices while the pressure field is approximated by a piecewise constant scalar function inside the diamond cells. In this formulation, the well-posedness of the method is achieved through a stabilization term $\grave{a}$ la Brezzi-Pitkaranta [15] in the mass conservation equation. Convergence analysis and a priori estimates for the two-dimensional method are available in [33].

The new DDFV method that we study in this work extends the discrete 2-D setting proposed in [33] for the steady Stokes equation with variable viscosity to the 3-D framework for polyhedral meshes of [18]. More precisely, the degrees of freedom of the components of the velocity are defined for the control volumes of the primal mesh, the dual mesh of the vertices, and the dual mesh of faces and edges. On its turn, the pressure variable is approximated by a piecewise constant function defined on the mesh of the diamond cells. We emphasize the fact that the present DDFV scheme is not a simple extension to three spatial dimensions of the 2-D scheme originally developed in [33], because it is based on a construction for the dual meshes and the diamond mesh that is very specific to the $3-\mathrm{D}$ case. For the present scheme, we prove a discrete analog of the Korn inequality, the uniform stability, the well-posedness and the convergence. Moreover, we derive a priori estimates for the degrees of freedom of velocity and pressure using suitably defined mesh dependent norms, and a priori estimates for the approximation errors in the continuous setting using standard Sobolev norms.

A remarkable fact of this DDFV method is that the flux balance equations can be reformulated through discrete divergence operators for discrete vector and tensor fields. These divergence operators satisfy several discrete duality relations, i.e. summation-by-parts formulas, that involve the discrete gradients and properly defined inner products for all the degrees of freedom. This fact was originally noted for the diffusion equation [24], the advection-diffusion equation [21], and the 2-D Stokes equation [33], and allows us to reinterpret the current DDFV method as a mimetic discretizations $[3,8,9,6,7,5,16,17,4]$.

The paper is organized as follows. In Section 2 we introduce the mathematical model. In Section 3 , we recall the general DDFV framework and formulate the stabilized scheme for the Stokes problem. In Section 5, we carry out the theoretical analysis by proving well-posedness and convergence of the method, and deriving a priori error estimates for the approximation of the vector and the scalar variable. For simplicity of exposition, we focus the presentation of the method in Section 3 and the theoretical analysis of Section 5 to the case of homogeneous boundary conditions. In Section 6 , we comment the performance of the scheme for a set of numerical experiments. In Section 7, we offer final remarks and conclusions.

\section{Steady Stokes equation}

We are concerned with the finite volume approximation on the computational domain $\Omega$ with boundary $\Gamma$ of the 3 -D steady Stokes problem with variable viscosity $\eta$ and loading term $f$ that reads as

$$
\begin{aligned}
\text { find }(\boldsymbol{u}, p) \in\left(H^{1}(\Omega)\right)^{3} \times L^{2}(\Omega) \text { such that: } & \\
\operatorname{div}(-2 \eta \mathrm{D}(\boldsymbol{u})+p \mathbb{I})=\boldsymbol{f} & \text { in } \Omega, \\
\operatorname{div}(\boldsymbol{u})=0 & \text { in } \Omega, \\
\boldsymbol{u}=0 & \text { on } \Gamma,
\end{aligned}
$$

where $\mathrm{D}(\boldsymbol{u})=\left(\nabla \boldsymbol{u}+(\nabla \boldsymbol{u})^{T}\right) / 2$. We refer to the vector variable $\boldsymbol{u}$ as the velocity and to the scalar variable $p$ as the pressure. Moreover, we assume that:

$\left(\mathrm{H}_{1}\right): \Omega$ is a bounded, open, polyhedral subset of $\mathfrak{R}^{3}$ with Lipschitz boundary $\Gamma$; 
$\left(\mathrm{H}_{2}\right): \eta: \bar{\Omega} \rightarrow \mathfrak{R}$ is a uniformly bounded, positive, Lipschitz continuous function;

$\left(\mathrm{H}_{3}\right): \boldsymbol{f} \in\left(L^{2}(\Omega)\right)^{3}$.

Assumptions $\left(\mathrm{H}_{2}\right)$ implies that there exists a positive constant number $C_{\eta}$ such that

$$
\left|\eta(\boldsymbol{x})-\eta\left(\boldsymbol{x}^{\prime}\right)\right| \leq C_{\eta}\left|\boldsymbol{x}-\boldsymbol{x}^{\prime}\right| \text { for almost every } \boldsymbol{x}, \boldsymbol{x}^{\prime} \in \bar{\Omega},
$$

and two positive constant numbers $\underline{C}_{\eta}$ and $\bar{C}_{\eta}$ such that

$$
\underline{C}_{\eta} \leq \eta \leq \bar{C}_{\eta}, \quad \text { almost everywhere in } \Omega .
$$

Existence and uniqueness of solution fields $(\boldsymbol{u}, p)$ are guaranteed by taking the additional condition for the pressure that:

$$
\int_{\Omega} p(x) d V=0
$$

The well-posedness of this mathematical model is discussed in several books, see, for example [11].

\section{Discrete Duality Finite Volume method}

We have chosen the 3-D framework of [18], we briefly present the construction of the different meshes.

\subsection{Mesh constructions}

The formulation of the DDFV method requires the triplet of meshes $\mathfrak{M}_{h}^{\mathcal{T}}:=\left(\mathfrak{M}_{h}^{\mathcal{P}}, \mathfrak{M}_{h}^{\mathcal{V}}, \mathfrak{M}_{h}^{\mathcal{E}}\right)$ and the mesh of diamond cells $\mathfrak{M}_{h}^{\mathcal{D}}$. The construction of $\mathfrak{M}_{h}^{\mathcal{T}}$ and $\mathfrak{M}_{h}^{\mathcal{D}}$ and the presentation of their properties are the topics of this subsection. For simplicity, we restrict the description to convex polyhedrons. The mesh construction starts from $\mathfrak{M}_{h}^{\mathcal{P}}$, the primary partition of the computational domain $\Omega$. We denote

- the set of mesh polyhedrons by $\mathcal{P}$, a polyhedron by $\mathrm{p}$, its three-dimensional measure, i.e., the volume, by $m_{\mathrm{p}}$, the coordinate vector of its barycenter by $\boldsymbol{x}_{\mathrm{p}}$;

- the set of mesh faces by $\mathcal{F}$, a face by $f$, its two-dimensional measure, i.e., the area, by $|\mathfrak{f}|$, the coordinate vector of its barycenter by $\boldsymbol{x}_{\mathrm{f}}$;

- the set of mesh edges by $\mathcal{E}$, an edge by e, its one-dimensional measure, i.e., the length, by $|\mathrm{e}|$, the coordinate vector of its midpoint by $\boldsymbol{x}_{\mathrm{e}}$;

- the set of mesh vertices by $\mathcal{V}$, a vertex by $v$ and its coordinate vector by $\boldsymbol{x}_{\mathrm{v}}$.

The cells of mesh $\mathfrak{M}_{h}^{\mathcal{V}}$ are associated to the vertices of $\mathcal{V}$, while the cells of mesh $\mathfrak{M}_{h}^{\mathcal{E F}}$ are associated to the edges of $\mathcal{E}$ and to the faces of $\mathcal{F}$. For this reason, they are referred to as cells of type vertex, and cells of type edge and face, respectively. Since there is a bijective correspondance between mesh vertices and cells of type vertex, we will use the same vertex symbol $v$ to denote them. We denote the three-dimensional measure (volume) of the vertex cell $\vee$ by $m_{\mathrm{v}}$. We also denote both cells of type edge and face, which concur in the definition of mesh $\mathfrak{M}_{h}^{\mathcal{E F}}$, by the generic symbol s; consistently, $m_{\mathrm{s}}$ denotes the volume of the generic cell $s \in \mathfrak{M}_{h}^{\mathcal{E F}}$. The symbols $\mathrm{p}, \mathrm{v}, \mathrm{s}$ may be conveniently sub-indexed to denote different instances, e.g., $\mathrm{p}_{1}, \mathrm{p}_{2}, \mathrm{v}_{\mathrm{A}}, \mathrm{v}_{\mathrm{B}}$, etc. The sub-index $h$ that labels $\mathfrak{M}_{h}^{\mathcal{T}}$ and $\mathfrak{M}_{h}^{\mathcal{D}}$ is the mesh size, i.e., a characteristic length of the mesh, and is defined as usual by $h=\max _{\mathrm{e} \in \mathcal{E}}|\mathrm{e}|$. 


\section{Construction of $\mathfrak{M}_{h}^{\mathcal{D}}$}

The diamond mesh is obtained by a decomposition of the mesh polyhedrons of $\mathfrak{M}_{h}^{\mathcal{P}}$ following the next two steps. In the first step, we split each mesh face $f$ into a set of triangles by connecting each vertex of face $f$ to $\boldsymbol{x}_{\boldsymbol{f}}$; in the second step, we connect each triangle of face $f$ to $\boldsymbol{x}_{\mathrm{p}}$ the barycenter of the polyhedrons $\mathrm{p}$ to which that face belongs. If the face is internal, i.e., it is shared by two distinct cells of $\mathfrak{M}_{h}^{\mathcal{P}}$, this construction provides two tetrahedral cells for each edge of the face. These two tetrahedrons are located on the opposite side of the face as shown in Fig. 1- $(a)$ and their union is an internal diamond cell. If the face is on the boundary of $\Omega$, this construction provides a single tetrahedron, a boundary diamond cell, for each edge of the face. The collection of internal and boundary diamond cells forms the mesh $\mathfrak{M}_{h}^{\mathcal{D}}$. We denote the generic cell of $\mathfrak{M}_{h}^{\mathcal{D}}$ by $\mathrm{D}$, its three-dimensional measure by $m_{\mathrm{D}}$, and its diameter by $h_{\mathrm{D}}$.

By construction, there is a bijective correspondance between the diamond cells in $\mathfrak{M}_{h}^{D}$ and the ordered pairs "(edge, face)" denoted by (e,f) and such that $\mathrm{e} \in \partial \mathrm{f}$. We refer to such a combination by the wording admissible pair and denote the corresponding diamond cell by $\mathrm{D}_{(\mathrm{e}, \mathrm{f})}$. For any diamond cell $\mathrm{D}_{(\mathrm{e}, \mathrm{f})} \in \mathfrak{M}_{h}^{D}$ associated to the admissible pair $(\mathrm{e}, \mathrm{f})$ we consider the seven geometric points:

- $\mathrm{F}=\boldsymbol{x}_{\mathrm{f}}$, the barycenter of face $\mathrm{f} ; \mathrm{E}$, the midpoint of edge e;

- A, the first vertex of edge e; B, the second vertex of edge e;

- $\mathrm{K}=\boldsymbol{x}_{\mathrm{p}}$, the barycenter of the cell to which face $\mathrm{f}$ belongs; $\mathrm{L}$, the barycenter of the second cell to which face $f$ belongs when $f$ is an internal face. If $f$ is a boundary face, we take $L=F$;

- D, the barycenter of the triangle whose vertices are the points $\mathrm{F}, \mathrm{A}$ and $\mathrm{B}$.

Face and edge orientations are chosen in accordance with the following criteria. When $f$ is a boundary face, the unit vector $\mathbf{n}_{\mathrm{f}}$ orthogonal to $\mathrm{f}$ always points out of domain $\Omega$, while, when $\mathrm{f}$ is an internal face, $\mathbf{n}_{\mathrm{f}}$ is positively oriented from point $\mathrm{K}$ toward point $\mathrm{L}$. Likewise, we assume that the unit vector parallel to the direction of edge e is oriented from vertex A toward vertex B.

Despite the redundancy of notation, it is useful to denote the coordinate vectors of these seven points by $\boldsymbol{x}$ sub-indexed by the point's label; for example, $\boldsymbol{x}_{\mathrm{F}} \equiv \boldsymbol{x}_{\mathrm{f}}$ is the coordinate vector of the geometric point $F$, etc. In the rest of the section, the symbol $\mathcal{T}_{v_{1} v_{2} v_{3}}$ will denote the triangle whose vertices are $v_{1}, v_{2}$, and $v_{3}$, these latters being any triple combination without repetition of the seven geometric points defined above. We will also use the notation $\operatorname{HULL}\{\star\}$ to indicate the convex hull of the set of points denoted by $\star$.

The geometric construction of the cells in $\mathfrak{M}_{h}^{\mathcal{P}}, \mathfrak{M}_{h}^{\mathcal{V}}$, and $\mathfrak{M}_{h}^{\mathcal{E F}}$ is based on three different decompositions and re-assembly of the diamond cells of $\mathfrak{M}_{h}^{\mathcal{D}}$. Any diamond cell can, indeed, be split into two subcells in three different ways, each one of which leads to one of the meshes of the triplet $\mathfrak{M}_{h}^{\mathcal{T}}$. We also use the symbol $\mathfrak{M}_{h \mid \nu}^{\mathcal{D}}$ where $\nu$ is the control volume associated to one of the point of the set $\{\mathrm{A}, \mathrm{B}, \mathrm{E}, \mathrm{F}, \mathrm{K}, \mathrm{L}\}$ or one of the control volumes $\mathrm{p}, \mathrm{v}, \mathrm{s}$ to denote the subset of $\mathfrak{M}_{h}^{\mathcal{D}}$ of all the diamond cells $\mathrm{D}_{(\mathrm{e}, \mathrm{f})}$ such that $m_{\nu \cap \mathrm{D}_{(\mathrm{e}, \mathrm{f})}}>0$. Likewise, $\mathfrak{M}_{h \mid \mathrm{D}}^{\mathcal{D}}$ denotes the set of diamonds $\mathrm{D}^{\prime}$ that are adjacent to $\mathrm{D}$ and such that the surface $\sigma=\overline{\mathrm{D}} \cap \overline{\mathrm{D}^{\prime}}$. We also use notation $\left(\mathrm{D} \mid \mathrm{D}^{\prime}\right)$ and $\sigma=\left(\mathrm{D} \mid \mathrm{D}^{\prime}\right)$ to denote such pairs $\left(\mathrm{D}, \mathrm{D}^{\prime}\right)$ in $\in \mathfrak{M}_{h}^{\mathcal{D}} \times \mathfrak{M}_{h}^{\mathcal{D}}$.

Remark 1 The mesh construction described in the next paragraphs always takes place, even for boundary items. Nonetheless, in such a case, point $\mathrm{L}$ may coincide with point $\mathrm{F}$, and several triangular subsurfaces that are introduced below may degenerate into a surface with zero two-dimensional measure.

\section{Scheme implementation}

Despite the apparent complexity of the mesh construction that follows in the next paragraphs, the practical implementation of this method in a software program can be easily managed. In fact, only information from the connectivity structure of mesh $\mathfrak{M}_{h}^{\mathcal{P}}$ is really required. More precisely, we can exploit the correspondance between any admissible pair of type (edge,face) and a diamond cell of mesh 
$\mathfrak{M}_{h}^{\mathcal{D}}$, to construct local data structures for all the diamonds that are sequentially referenced in the loop on all the edges of a face, for all the faces of $\mathfrak{M}_{h}^{\mathcal{P}}$. It will be clear from the scheme formulation discussed in the next section, that all global operators such as discrete gradient and divergence can be built by assemblying these local contributions.

\section{Characterization of $\mathfrak{M}_{h}^{\mathcal{P}}$}

$\mathfrak{M}_{h}^{\mathcal{P}}$ is the primary mesh from which all mesh construction starts and its control volumes are the polyhedrons considered at the beginning of the section. Here, we discuss the connection between $\mathfrak{M}_{h}^{\mathcal{P}}$ and $\mathfrak{M}_{h}^{D}$, i.e., between the primary cells and the diamond cells, and we introduce some additional notation.

If $\mathrm{f}$ is an internal face, we consider the situation shown in Fig. 1- $(a)$, where $\mathrm{f}$ is shared by the primary cells $p_{K}$ and $p_{L}$. If $f$ is a boundary face, we assume that it belongs to the primary cell $p_{K}$, and all considerations concerning cell $\mathrm{p}_{\mathrm{L}}$ are to be dropped out. As shown in Fig. 1-(b), for any admissible pair $(e, f)$ we consider the surface given by the union of the four triangles indicated below:

$$
\mathcal{S}_{\mathrm{D}, \mathrm{KL}}=\mathcal{T}_{\mathrm{DFA}} \cup \mathcal{T}_{\mathrm{DAE}} \cup \mathcal{T}_{\mathrm{DEB}} \cup \mathcal{T}_{\mathrm{DBF}},
$$

which is in the interior of the diamond cell $\mathrm{D}_{(\mathrm{e}, \mathrm{f})}$. Using surface $\mathcal{S}_{\mathrm{D}, \mathrm{KL}}$, we can reconstruct primary cells labeled by $\mathrm{K}$ and $\mathrm{L}$ :

$$
\mathrm{p}_{\mathrm{K}}=\cup_{\mathrm{D} \in \mathfrak{M}_{h \mid \mathrm{K}}^{\mathcal{D}}} \operatorname{HULL}\left\{\mathrm{K}, \mathcal{S}_{\mathrm{D}, \mathrm{KL}}\right\} \quad \text { and } \quad \mathrm{p}_{\mathrm{L}}=\cup_{\mathrm{D} \in \mathfrak{M}_{h \mid \mathrm{L}}^{\mathcal{D}}} \operatorname{HULL}\left\{\mathrm{L}, \mathcal{S}_{\mathrm{D}, \mathrm{KL}}\right\} .
$$

Finally, we introduce the surface vector $\mathbf{N}_{\mathrm{K}, \mathrm{L}}$ that is given by summing the vector products related to the four triangles in (7) through the formula:

$$
\mathbf{N}_{\mathrm{K}, \mathrm{L}}=\frac{1}{2} \overrightarrow{\mathrm{AB}} \times \overrightarrow{\mathrm{EF}}
$$

Its orientation is such that $\mathbf{N}_{\mathrm{K}, \mathrm{L}} \cdot \overrightarrow{\mathrm{KL}}=3\left|\mathrm{D}_{(\mathrm{e}, \mathrm{f})}\right|>0$.

\section{Construction of $\mathfrak{M}_{h}^{\nu}$}

In this paragraph we explain the construction of the node mesh $\mathfrak{M}_{h}^{\mathcal{V}}$ whose cells are associated to $\mathcal{V}$, the vertices of mesh $\mathfrak{M}_{h}^{\mathcal{P}}$. As shown in Fig. 1- $(c)$, for any admissible pair $(\mathrm{e}, \mathrm{f})$ we consider the surface given by the union of the four triangles indicated below:

$$
\mathcal{S}_{\mathrm{D}, \mathrm{AB}}=\mathcal{T}_{\mathrm{DKF}} \cup \mathcal{T}_{\mathrm{DFL}} \cup \mathcal{T}_{\mathrm{DLE}} \cup \mathcal{T}_{\mathrm{DEK}},
$$

which is in the interior of the diamond cell $D_{(e, f)}$. Using this surface, we construct the dual cells associated to $\mathrm{A}$ and $\mathrm{B}$

$$
\mathrm{v}_{\mathrm{A}}=\cup_{\mathrm{D} \in \mathfrak{M}_{h}^{\mathcal{D}}} \operatorname{HULL}\left\{\mathrm{A}, \mathcal{S}_{\mathrm{D}, \mathrm{AB}}\right\} \quad \text { and } \quad \mathrm{v}_{\mathrm{B}}=\cup_{\mathrm{D} \in \mathfrak{M}_{h}^{\mathcal{D}}} \operatorname{HULL}\left\{\mathrm{B}, \mathcal{S}_{\mathrm{D}, \mathrm{AB}}\right\} .
$$

We denote this dual mesh by $\mathfrak{M}_{h}^{\mathcal{V}}$ and an example of its dual cell for a primary mesh formed by cubic cells is given in Fig. 2, plot (a). We also introduce the surface vector $\mathbf{N}_{\mathrm{A}, \mathrm{B}}$ :

$$
\mathbf{N}_{\mathrm{A}, \mathrm{B}}=\frac{1}{2} \overrightarrow{\mathrm{EF}} \times \overrightarrow{\mathrm{KL}}
$$

Its orientation is such that $\mathbf{N}_{\mathrm{A}, \mathrm{B}} \cdot \overrightarrow{\mathrm{AB}}=3\left|\mathrm{D}_{(\mathrm{e}, \mathrm{f})}\right|>0$.

\section{Construction of $\mathfrak{M}_{h}^{\mathcal{E F}}$}

In this paragraph we explain the construction of the edge-face mesh $\mathfrak{M}_{h}^{\mathcal{E F}}$ whose cells are associated 
to $\mathcal{E}$ and $\mathcal{F}$, the edges and faces of mesh $\mathfrak{M}_{h}^{\mathcal{P}}$, respectively. As shown in Fig. 1- $(d)$, for any admissible pair $(e, f)$ we consider the surface given by the union of the four triangles indicated below:

$$
\mathcal{S}_{\mathrm{D}, \mathrm{EF}}=\mathcal{T}_{\mathrm{DKA}} \cup \mathcal{T}_{\mathrm{DAL}} \cup \mathcal{T}_{\mathrm{DLB}} \cup \mathcal{T}_{\mathrm{DBK}}
$$

Using this surface, we construct the dual cells associated to E and F:

$$
\mathrm{s}_{\mathrm{E}}=\cup_{\mathrm{D}_{(\mathrm{e}, \mathrm{f})} \in \mathfrak{M}_{h}^{\mathcal{D}}} \operatorname{HULL}\left\{\mathrm{E}, \mathcal{S}_{\mathrm{D}, \mathrm{EF}}\right\} \quad \text { and } \quad \mathrm{s}_{\mathrm{F}}=\cup_{\mathrm{D}_{(\mathrm{e}, \mathrm{f})} \in \mathfrak{M}_{h}^{\mathcal{D}}} \operatorname{HULL}\left\{\mathrm{F}, \mathcal{S}_{\mathrm{D}, \mathrm{EF}}\right\}
$$

An example of two dual cells of $\mathfrak{M}_{h}^{\mathcal{E F}}$ of type face and edge for a primary mesh formed by cubic cells is given in Fig. 2, e.g., plots $(b)$ and $(c)$, respectively. Finally, we introduce the surface vector $\mathbf{N}_{\mathrm{E}, \mathrm{F}}$ :

$$
\mathbf{N}_{\mathrm{E}, \mathrm{F}}=\frac{1}{2} \overrightarrow{\mathrm{KL}} \times \overrightarrow{\mathrm{AB}}
$$

Its orientation is such that $\mathbf{N}_{\mathrm{E}, \mathrm{F}} \cdot \overrightarrow{\mathrm{EF}}=3\left|\mathrm{D}_{(\mathrm{e}, \mathrm{f})}\right|>0$.

Finally, we introduce the auxiliary notation for the surface vectors:

$$
\mathbf{N}_{\mathrm{D}, \mathrm{p}}=\left\{\begin{array}{ll}
+\mathbf{N}_{\mathrm{K}, \mathrm{L}} & \text { if } \mathrm{p} \equiv \mathrm{p}_{\mathrm{K}} \\
-\mathbf{N}_{\mathrm{K}, \mathrm{L}} & \text { if } \mathrm{p} \equiv \mathrm{p}_{\mathrm{L}}
\end{array}, \quad \mathbf{N}_{\mathrm{D}, \mathrm{v}}=\left\{\begin{array}{ll}
+\mathbf{N}_{\mathrm{A}, \mathrm{B}} & \text { if } \mathrm{v} \equiv \mathrm{v}_{\mathrm{A}} \\
-\mathbf{N}_{\mathrm{A}, \mathrm{B}} & \text { if } \mathrm{v} \equiv \mathrm{v}_{\mathrm{B}}
\end{array}, \quad \mathbf{N}_{\mathrm{D}, \mathrm{s}}= \begin{cases}+\mathbf{N}_{\mathrm{E}, \mathrm{F}} & \text { if } \mathrm{s} \equiv \mathrm{s}_{\mathrm{E}} \\
-\mathbf{N}_{\mathrm{E}, \mathrm{F}} & \text { if } \mathrm{s} \equiv \mathrm{s}_{\mathrm{F}}\end{cases}\right.\right.
$$

where $\mathrm{D} \equiv \mathrm{D}_{(\mathrm{e}, \mathrm{f})}$ is the diamond cell associated to the pair $(\mathrm{e}, \mathrm{f})$ and $\mathrm{A}, \mathrm{B}, \mathrm{K}, \mathrm{L}, \mathrm{E}, \mathrm{F}$ are the corresponding auxiliary points. This notation will be used in the definition of the discrete divergence operator of the following subsection.

\section{Mesh Regularity}

We are interested in the formulation of an approximation method based on a family of meshes $\left\{\left(\mathfrak{M}_{h}^{\mathcal{T}}, \mathfrak{M}_{h}^{\mathcal{D}}\right)\right\}$ for $h \rightarrow 0$. We will take the few minimal assumptions in order to avoid some pathological situations that may occur in the refinement process.

$\left(\mathrm{A}_{1}\right):$ All the primary partitions $\mathfrak{M}_{h}^{\mathcal{P}}$ for $h \rightarrow 0$ are such that:

$$
\left(A_{11}\right) \bar{\Omega}=\cup_{p \in \mathcal{P}} \bar{p}
$$

$\left(A_{12}\right)$ each polyhedron face is either an interface between two distinct polyhedrons or a boundary face; therefore, if $\mathrm{f}$ is a face of $\mathcal{F}$ either there exist two polyhedrons $\mathrm{p}_{1}$ and $\mathrm{p}_{2}$ in $\mathcal{P}$ such that $\mathrm{f}=\overline{\mathrm{p}_{1}} \cap \overline{\mathrm{p}_{2}}$, or there exists a polyhedron $\mathrm{p}_{1}$ in $\mathcal{P}$ such that $\mathrm{f}=\partial \mathrm{p}_{1} \cap \partial \Omega$;

$\left(A_{13}\right)$ every edge of a face of $\mathcal{F}$ is an edge of $\mathcal{E}$;

$\left(\mathrm{A}_{14}\right)$ every vertex of a face of $\mathcal{F}$ is a vertex of $\mathcal{V}$.

$\left(\mathrm{A}_{2}\right)$ : There exist two positive integer numbers $\mathcal{N}_{\mathcal{E F}}$ and $\mathcal{N}_{\mathcal{V}}$ such that:

$\left(A_{21}\right)$ : the number of faces of each polyhedral cell $p \in \mathcal{P}$ and the number of edges of each polyhedral face $\mathrm{f} \in \mathcal{F}$ are uniformly bounded by $\mathcal{N}_{\mathcal{E} \mathcal{F}}$ for $h \rightarrow 0$;

$\left(A_{22}\right)$ : the number of edges incident to any vertex is uniformly bounded by $\mathcal{N}_{\mathcal{V}}$ for $h \rightarrow 0$.

From Assumptions $\left(A_{1}\right)-\left(A_{2}\right)$ there follow that:

$\left(\mathrm{M}_{1}\right)$ : there exists a positive integer number $\mathcal{N}_{*}$, which is independent of $h$, such that $\mathcal{S}_{h \mid \mathrm{p}}$, the decomposition of every polyhedron $\mathrm{p} \in \mathfrak{M}_{h}^{\mathcal{P}}$ into tetrahedrons, is formed by at most $\mathcal{N}_{*}$ tetrahedrons; 
$\left(\mathrm{M}_{2}\right)$ : all relevant geometric quantities of the three meshes forming $\mathfrak{M}_{h}^{\mathcal{T}}$ and mesh $\mathfrak{M}_{h}^{\mathcal{D}}$ scale consistently. In particular, there exists a constant $C_{r e g}$ independent of $h$ such that

$$
\begin{aligned}
& \forall \mathrm{e} \in \mathcal{E}: C_{r e g} h \leq|\mathrm{e}|, \quad \forall \mathrm{f} \in \mathcal{F}: C_{r e g}^{2} h^{2} \leq|\mathrm{f}|, \quad \forall \mathrm{p} \in \mathcal{P}: C_{r e g}^{3} h^{3} \leq m_{\mathrm{p}} ; \\
& \forall \sigma \subset \partial \mathrm{D} \cup \mathcal{S}_{\mathrm{D}, \mathrm{KL}} \cup \mathcal{S}_{\mathrm{D}, \mathrm{AB}} \cup \mathcal{S}_{\mathrm{D}, \mathrm{EF}}, \forall \mathrm{D} \in \mathfrak{M}_{h}^{\mathcal{D}}: C_{r e g}^{2} h^{2} \leq|\sigma| \leq h^{2} ; \\
& \forall \mathrm{D} \in \mathfrak{M}_{h}^{\mathcal{D}}: C_{r e g} h \leq h_{\mathrm{D}} \leq h \text { and } C_{r e g}^{3} h^{3} \leq m_{\mathrm{D}} ; \\
& \forall \nu \in \mathfrak{M}_{h}^{\mathcal{P}}, \mathfrak{M}_{h}^{\mathcal{V}}, \mathfrak{M}_{h}^{\mathcal{E F}}: \operatorname{diam}(\nu) \leq C_{r e g} h .
\end{aligned}
$$

$\left(\mathrm{M}_{3}\right)$ : there exists a real positive constant $C_{A g}$, which is independent of the diamond cell $\mathrm{D} \in \mathfrak{M}_{h}^{\mathcal{D}}$ and mesh size $h$, such that for any function $v \in H^{1}(\mathrm{D})$ there holds that

$$
\begin{aligned}
\forall \sigma \subset \partial \mathrm{D} \cup \mathcal{S}_{\mathrm{D}, \mathrm{KL}} \cup \mathcal{S}_{\mathrm{D}, \mathrm{AB}} \cup \mathcal{S}_{\mathrm{D}, \mathrm{EF}}, \forall \mathrm{D} \in \mathfrak{M}_{h}^{\mathcal{D}}: \\
\|v\|_{L^{2}(\sigma)}^{2} \leq C_{A g}\left(h_{\mathrm{D}}^{-1}\|v\|_{L^{2}(\mathrm{D})}^{2}+h_{\mathrm{D}}|v|_{H^{1}(\mathrm{D})}^{2}\right) .
\end{aligned}
$$

We will refer to (18) as the Agmon inequality.

\subsection{Degrees of freedom, interpolations and discrete operators}

\section{Degrees of freedom}

Using $\mathfrak{M}_{h}^{\mathcal{T}}$ and $\mathfrak{M}_{h}^{\mathcal{D}}$, we define several different types of degrees of freedom to represent scalar, vector, and tensor fields of the continuum setting in the discrete setting. More precisely, we consider:

- one number per cell of the meshes $\mathfrak{M}_{h}^{\mathcal{P}}, \mathfrak{M}_{h}^{\mathcal{V}}, \mathfrak{M}_{h}^{\mathcal{E F}}$ to define the linear space of the discrete scalar fields on $\mathfrak{M}_{h}^{\mathcal{T}}$, which is denoted by $\mathfrak{T}_{h}$;

- one number per cell of the mesh $\mathfrak{M}_{h}^{\mathcal{D}}$ to define the linear space of the discrete scalar fields on $\mathfrak{M}_{h}^{\mathcal{D}}$, which is denoted by $\mathfrak{D}_{h}$;

- one three-dimensional vector per cell of the meshes $\mathfrak{M}_{h}^{\mathcal{P}}, \mathfrak{M}_{h}^{\mathcal{V}}, \mathfrak{M}_{h}^{\mathcal{E F}}$ to define the linear space of the discrete three-dimensional vector fields on $\mathfrak{M}_{h}^{\mathcal{T}}$, which is denoted by $\mathfrak{T}_{h}^{3}$;

- one three-dimensional vector per cell of the mesh $\mathfrak{M}_{h}^{\mathcal{D}}$ to define the linear space of the discrete three-dimensional vector fields on $\mathfrak{M}_{h}^{\mathcal{D}}$, which is denoted by $\mathfrak{D}_{h}^{3}$;

- one $3 \times 3$-sized matrix per cell of the mesh $\mathfrak{M}_{h}^{\mathcal{D}}$ to define the linear space of the discrete $3 \times 3$ tensor fields on $\mathfrak{M}_{h}^{\mathcal{D}}$, which is denoted by $\mathfrak{D}_{h}^{3 \times 3}$.

We also introduce $\mathfrak{T}_{h, 0}$ and $\mathfrak{T}_{h, 0}^{3}$, which are, respectively, the linear subspace of the discrete scalar fields in $\mathfrak{T}_{h}$ and vector fields in $\mathfrak{T}_{h}^{3}$ whose boundary degrees of freedom are zero.

The geometric cells to which each degree of freedom is attached is denoted by a cell's sub-index; for example, $q \in \mathfrak{T}_{h}$ means that $q=\left\{\left(q_{\mathrm{p}}\right)_{\mathrm{p} \in \mathfrak{M}_{h}^{\mathcal{P}}},\left(q_{\mathrm{v}}\right)_{\mathrm{v} \in \mathfrak{M}_{h}^{\nu}},\left(q_{\mathrm{s}}\right)_{\left.\mathbf{s} \in \mathfrak{M}_{h}^{\mathcal{F}}\right\}}\right.$, where $q_{\mathrm{p}}$ is the number attached to cell $\mathrm{p}$, etc. We make also use of the simplified notation $u_{\mathrm{K}}, u_{\mathrm{A}}$, etc to denote the degree of freedom associated to the cells $\mathrm{p}_{\mathrm{K}}, \mathrm{v}_{\mathrm{A}}$, etc. Now, we define the discrete operators that act on the linear spaces of the degrees of freedom introduced above.

\section{Discrete gradient operators}

The discrete gradient is formally given by the operator $\nabla_{h}: \mathfrak{T}_{h}^{3} \rightarrow \mathfrak{D}_{h}^{3 \times 3}$, when applied to the discrete vectors of $\mathfrak{T}_{h}^{3}$. The discrete gradient of the vector field $\boldsymbol{v} \in \mathfrak{T}_{h}^{3}$ is given by $\nabla_{h} \boldsymbol{v}:=\left\{\left(\nabla_{h}^{\mathrm{D}} \boldsymbol{v}\right)_{\mathrm{D} \in \mathfrak{M}_{h}^{D}}\right\}$ with

$$
\forall \mathrm{D} \in \mathfrak{M}_{h}^{\mathcal{D}}: \nabla_{h}^{\mathrm{D}} \boldsymbol{v}:=\frac{1}{3 m_{\mathrm{D}}}\left(\left(\boldsymbol{v}_{\mathrm{L}}-\boldsymbol{v}_{\mathrm{K}}\right) \otimes \mathbf{N}_{\mathrm{K}, \mathrm{L}}+\left(\boldsymbol{v}_{\mathrm{B}}-\boldsymbol{v}_{\mathrm{A}}\right) \otimes \mathbf{N}_{\mathrm{A}, \mathrm{B}}+\left(\boldsymbol{v}_{\mathrm{F}}-\boldsymbol{v}_{\mathrm{E}}\right) \otimes \mathbf{N}_{\mathrm{E}, \mathrm{F}}\right),
$$

where $\{\mathrm{K}, \mathrm{L}, \mathrm{A}, \mathrm{B}, \mathrm{E}, \mathrm{F}\}, \mathbf{N}_{\mathrm{K}, \mathrm{L}}, \mathbf{N}_{\mathrm{A}, \mathrm{B}}, \mathbf{N}_{\mathrm{E}, \mathrm{F}}$ are the six points and the three surface vectors defined for $\mathrm{D}$ by the geometric construction of subsection 3.1 and $\otimes$ is the tensor product. 


\section{Discrete divergence operators}

The divergence is given by the operator $\operatorname{div}_{h}: \mathfrak{D}_{h}^{3 \times 3} \rightarrow \mathfrak{T}_{h}^{3}$ when applied to the tensor fields of $\mathfrak{D}_{h}^{3 \times 3}$. To this purpose, let us first recall that $\mathbf{N}_{D, p}, \mathbf{N}_{D, v}$, and $\mathbf{N}_{D, s}$ denote the vectors defined in (13), and that $\mathfrak{M}_{h \mid \mathrm{p}}^{\mathcal{D}}, \mathfrak{M}_{h \mid \mathrm{v}}^{\mathcal{D}}$, and $\mathfrak{M}_{h \mid \mathrm{s}}^{\mathcal{D}}$ denote the subsets of $\mathfrak{M}_{h}^{\mathcal{D}}$ formed by the diamond cells whose intersection with the cells $\mathrm{p} \in \mathfrak{M}_{h}^{\mathcal{P}}, \mathbf{v} \in \mathfrak{M}_{h}^{\mathcal{V}}$, and $\mathrm{s} \in \mathfrak{M}_{h}^{\mathcal{E} F}$, respectively, has a non-zero three-dimensional measure. The divergence of the tensor field $\psi \in \mathfrak{D}_{h}^{3 \times 3}$ is given by the triplet

$$
\operatorname{div}_{h}(\psi)=\left(\operatorname{div}_{h}^{\mathcal{P}}(\psi), \operatorname{div}_{h}^{\mathcal{V}}(\psi), \operatorname{div}_{h}^{\mathcal{E F}}(\psi)\right)
$$

with the following definitions

$$
\begin{aligned}
& \operatorname{div}_{h}^{\mathcal{P}}(\psi)=\left\{\left(\operatorname{div}_{h}^{\mathrm{p}}(\psi)\right)_{\mathbf{p} \in \mathfrak{M}_{h}^{\mathcal{P}}}\right\} \text { where } \forall \mathrm{p} \in \mathfrak{M}_{h}^{\mathcal{P}}: \operatorname{div}_{h}^{\mathrm{p}}(\psi)=\frac{1}{m_{\mathrm{p}}} \sum_{\mathbf{D} \in \mathfrak{M}_{h}^{\mathcal{D}}} \psi_{\mathrm{D}} \cdot \mathbf{N}_{\mathrm{D}, \mathrm{p}}, \\
& \operatorname{div}_{h}^{\mathcal{V}}(\psi)=\left\{\left(\operatorname{div}_{h}^{v}(\psi)\right)_{\mathbf{v} \in \mathfrak{M}_{h}^{\nu}}\right\} \text { where } \forall \mathbf{v} \in \mathfrak{M}_{h}^{\mathcal{V}}: \operatorname{div}_{h}^{v}(\psi)=\frac{1}{m_{\mathrm{v}}} \sum_{\left.\mathbf{D} \in \mathfrak{M}_{h}^{D}\right|_{\mathrm{v}}} \psi_{\mathbf{D}} \cdot \mathbf{N}_{\mathrm{D}, \mathrm{v}}, \\
& \operatorname{div}_{h}^{\mathcal{E F}}(\psi)=\left\{\left(\operatorname{div}_{h}^{\mathbf{s}}(\psi)\right)_{\mathbf{s} \in \mathfrak{M}_{h}^{\mathcal{E}}}\right\} \text { where } \forall \mathbf{s} \in \mathfrak{M}_{h}^{\mathcal{E F}}: \operatorname{div}_{h}^{\mathbf{s}}(\psi)=\frac{1}{m_{\mathbf{s}}} \sum_{\mathrm{D} \in \mathfrak{M}_{h}^{\mathcal{D}}} \psi_{\mathrm{D}} \cdot \mathbf{N}_{\mathrm{D}, \mathrm{s}} .
\end{aligned}
$$

Moreover, in the formulation of the DDFV method we use the internal divergence operator

$$
\operatorname{div}_{h}^{\text {int }}(\psi)=\left(\operatorname{div}_{h}^{\mathcal{P}}(\psi), \operatorname{div}_{h}^{\mathcal{V}, \text { int }}(\psi), \operatorname{div}_{h}^{\mathcal{E F}, \text { int }}(\psi)\right)
$$

where both $\operatorname{div}_{h}^{\mathcal{V} \text {,int }}(\psi)$ and $\operatorname{div}_{h}^{\mathcal{E F} \text {,int }}(\psi)$ are defined as in (22)-(23), but only for the internal control volumes of $\mathfrak{M}_{h}^{\mathcal{V}}$ and $\mathfrak{M}_{h}^{\mathcal{E F}}$, i.e., those control volumes associated to the points of type A, B or E, F located at the domain boundary. Regarding this definition, it is worth mentioning that we do not need such a restriction for the points of type $\mathrm{K}, \mathrm{L}$ as $\mathrm{K}$ is always an internal point and $\mathrm{L}$ coincides with $\mathrm{F}$ when $f$ is on the boundary.

We will also find it useful to introduce the discrete divergence of the vector fields of $\mathfrak{T}_{h}^{3}$, which is formally denoted by the operator $\operatorname{div}_{h}^{\mathcal{D}}: \mathfrak{T}_{h}^{3} \rightarrow \mathfrak{D}_{h}$ and given by

$$
\forall \boldsymbol{v} \in \mathfrak{T}_{h}^{3}: \operatorname{div}_{h}^{\mathcal{D}}(\boldsymbol{v})=\left\{\left(\operatorname{div}_{h}^{\mathrm{D}}(\boldsymbol{v})\right)_{\mathrm{D} \in \mathfrak{M}_{h}^{\mathrm{D}}}\right\} \quad \text { where } \quad \operatorname{div}_{h}^{\mathrm{D}}(\boldsymbol{v})=\operatorname{Tr}\left(\nabla_{h}^{\mathrm{D}} \boldsymbol{v}\right) .
$$

Using the compact notation $\operatorname{Tr}(\phi)$ to denote the vector of $\mathfrak{D}_{h}^{3}$ such that $\operatorname{Tr}(\phi)_{\mid \mathrm{D}}=\operatorname{Tr}\left(\phi_{\mid \mathrm{D}}\right)$ for any $\phi \in \mathfrak{D}_{h}^{3 \times 3}$ allows us to rewrite definition (25) as

$$
\forall \boldsymbol{v} \in \mathfrak{T}_{h}^{3}: \operatorname{div}_{h}^{\mathcal{D}}(\boldsymbol{v})=\operatorname{Tr}\left(\nabla_{h} \boldsymbol{v}\right)
$$

\section{Discrete strain rate tensor}

The discrete strain rate tensor operator is formally given by $\mathrm{D}_{h}: \mathfrak{T}_{h}^{3} \rightarrow \mathfrak{D}_{h}^{3 \times 3}$ and is defined as

$$
\forall \boldsymbol{v} \in \mathfrak{T}_{h}^{3}: \mathrm{D}_{h}(\boldsymbol{v})=\frac{\nabla_{h} \boldsymbol{v}+\left(\nabla_{h} \boldsymbol{v}\right)^{T}}{2} .
$$

Non-consistent discrete Laplacian operator

A stabilization term is considered in the formulation of the DDFV scheme of the next section. This term is based on the discretization of the Laplacian operator over $\mathfrak{M}_{h}^{\mathcal{D}}$ given by

$$
\forall q \in \mathfrak{D}_{h}: \Delta_{h} q_{\mid \mathrm{D}}:=\frac{1}{m_{\mathrm{D}}} \sum_{\mathrm{D}^{\prime} \in \mathfrak{M}_{h \mid \mathrm{D}}^{\mathcal{D}}} \frac{h_{\mathrm{D}}+h_{\mathrm{D}^{\prime}}}{2}\left(q_{\mathrm{D}^{\prime}}-q_{\mathrm{D}}\right),
$$


where the summation is on the diamond cells of $\mathfrak{M}_{h \mid \mathrm{D}}^{\mathcal{D}}$, which, we recall, contains those diamonds $\mathrm{D}^{\prime}$ that are adjacent to $\mathrm{D}$ and such that the surface $\mathrm{D} \cap \mathrm{D}^{\prime}$ has a non-zero three-dimensional measure. It is worth noting that (28) is a non-consistent approximation of the Laplacian operator. In fact, a consistent approximation based on a two-point flux formula would require the mesh to verify an orthogonality constraint as, for example, in the case of admissible meshes [27].

\section{Approximation of viscosity field}

Let $\eta_{h}=\left\{\left(\eta_{\mathrm{D}}\right)_{\mathrm{D} \in \mathfrak{M}_{h}^{\mathcal{D}}}\right\} \in \mathfrak{D}_{h}$ be any first-order approximation of the scalar field $\eta$ that is piecewiseconstant on $\mathfrak{M}_{h}^{\mathcal{D}}$, so that there holds the estimate

$$
\forall \mathrm{D} \in \mathfrak{M}_{h}^{\mathcal{D}}: \sup _{\boldsymbol{x} \in \mathrm{D}}\left|\eta_{\mathrm{D}}-\eta(\boldsymbol{x})\right| \leq \widetilde{C}_{\eta} h_{\mathrm{D}}
$$

where $\widetilde{C}_{\eta}$ is a real positive constant independent of $h_{\mathrm{D}}$ (and D). In view of the regularity of $\eta$, cf. Assumption $\left(\mathrm{H}_{2}\right)$, we can take $\eta_{\mathrm{D}}=\left(1 / m_{\mathrm{D}}\right) \int_{\mathrm{D}} \eta d V$. If $\eta$ is enough regular, we can also consider the pointwise value $\eta_{\mathrm{D}}=\eta\left(\widetilde{\boldsymbol{x}}_{\mathrm{D}}\right)$ where $\widetilde{\boldsymbol{x}}_{\mathrm{D}}$ is a suitably chosen point inside the corresponding diamond cell D. However, we emphasize that the derivation of the theoretical results in Section 5 only depend on estimate (29). From initial Assumption $\left(\mathrm{H}_{2}\right)$ and using the same constants $\underline{C}_{\eta}$ and $\bar{C}_{\eta}$ of inequalities (5), we easily obtain that

$$
\forall \mathrm{D} \in \mathfrak{M}_{h}^{\mathcal{D}}: \underline{C}_{\eta} \leq \eta_{\mathrm{D}} \leq \bar{C}_{\eta}
$$

\section{Interpolations}

On $\mathfrak{M}_{h}^{\mathcal{T}}$ we consider:

- $v^{I}=\left(\mathbb{P}_{\mathrm{M}}^{\mathcal{P}}(v), \mathbb{P}_{\mathrm{M}}^{\mathcal{V}}(v), \mathbb{P}_{\mathrm{M}}^{\mathcal{E F}}(v)\right)$, the mean-valued interpolation of the integrable field $v$, given by

$$
\begin{array}{r}
\mathbb{P}_{\mathrm{M}}^{\mathcal{P}}(v)=\left\{\left(\mathbb{P}_{\mathrm{M}}^{\mathrm{p}}(v)\right)_{\mathrm{p} \in \mathfrak{M}_{h}^{\mathcal{P}}}\right\} \text { where } \forall \mathrm{p} \in \mathfrak{M}_{h}^{\mathcal{P}}: \mathbb{P}_{\mathrm{M}}^{\mathrm{p}}(v):=\frac{1}{m_{\mathrm{p}}} \int_{\mathrm{p}} v(\boldsymbol{x}) d V, \\
\mathbb{P}_{\mathrm{M}}^{\mathcal{V}}(v)=\left\{\left(\mathbb{P}_{\mathrm{M}}^{\mathrm{v}}(v)\right)_{\mathrm{v} \in \mathfrak{M}_{h}^{v}}\right\} \text { where } \forall \mathrm{v} \in \mathfrak{M}_{h}^{\mathcal{V}}: \mathbb{P}_{\mathrm{M}}^{\mathrm{v}}(v):=\frac{1}{m_{\mathrm{v}}} \int_{\mathrm{v}} v(\boldsymbol{x}) d V, \\
\mathbb{P}_{\mathrm{M}}^{\mathcal{E \mathcal { F }}}(v)=\left\{\left(\mathbb{P}_{\mathrm{M}}^{\mathrm{s}}(v)\right)_{\left.\mathrm{s} \in \mathfrak{M}_{h}^{\mathcal{E F}}\right\}} \text { where } \forall \mathrm{s} \in \mathfrak{M}_{h}^{\mathcal{E F}}: \mathbb{P}_{\mathrm{M}}^{\mathrm{s}}(v):=\frac{1}{m_{\mathrm{s}}} \int_{\mathrm{s}} v(\boldsymbol{x}) d V,\right.
\end{array}
$$

- $v^{J}=\left(\mathbb{P}_{\mathrm{C}}^{\mathcal{P}}(v), \mathbb{P}_{\mathrm{C}}^{\mathcal{V}}(v), \mathbb{P}_{\mathrm{C}}^{\mathcal{E F}}(v)\right)$, the center-valued interpolation of the continuous field $v$, given by

$$
\begin{array}{r}
\mathbb{P}_{\mathrm{C}}^{\mathcal{P}}(v)=\left\{\left(\mathbb{P}_{\mathrm{C}}^{\mathrm{p}}(v)\right)_{\left.\mathrm{p} \in \mathfrak{M}_{h}^{\mathcal{P}}\right\}} \text { where } \forall \mathrm{p} \in \mathfrak{M}_{h}^{\mathcal{P}}: \mathbb{P}_{\mathrm{C}}^{\mathrm{p}}(v):=v\left(\boldsymbol{x}_{\mathrm{p}}\right),\right. \\
\mathbb{P}_{\mathrm{C}}^{\mathcal{V}}(v)=\left\{\left(\mathbb{P}_{\mathrm{C}}^{\mathrm{v}}(v)\right)_{\mathrm{v} \in \mathfrak{M}_{h}^{\mathcal{V}}}\right\} \text { where } \forall \mathrm{v} \in \mathfrak{M}_{h}^{\mathcal{V}}: \mathbb{P}_{\mathrm{C}}^{\mathrm{v}}(v):=v\left(\boldsymbol{x}_{\mathrm{v}}\right), \\
\mathbb{P}_{\mathrm{C}}^{\mathcal{E F}}(v)=\left\{\left(\mathbb{P}_{\mathrm{C}}^{\mathrm{s}}(v)\right)_{\left.\mathrm{s} \in \mathfrak{M}_{h}^{\mathcal{E F}}\right\}} \text { where } \forall \mathrm{s} \in \mathfrak{M}_{h}^{\mathcal{F} F}: \mathbb{P}_{\mathrm{C}}^{\mathrm{s}}(v):=v\left(\boldsymbol{x}_{\mathrm{s}}\right) .\right.
\end{array}
$$

Mean-valued and center-valued interpolations of a scalar field $v$ are naturally extended to a vector field $\boldsymbol{v}$ by applying formulas (31)-(33) and (34)-(36) to each vector component, thus leading to expressions like $\boldsymbol{v}^{I}=\left(\mathbb{P}_{\mathrm{M}}^{\mathcal{P}}(\boldsymbol{v}), \mathbb{P}_{\mathrm{M}}^{\mathcal{V}}(\boldsymbol{v}), \mathbb{P}_{\mathrm{M}}^{\mathcal{E F}}(\boldsymbol{v})\right)$ and $\boldsymbol{v}^{J}=\left(\mathbb{P}_{\mathrm{C}}^{\mathcal{P}}(\boldsymbol{v}), \mathbb{P}_{\mathrm{C}}^{\mathcal{V}}(\boldsymbol{v}), \mathbb{P}_{\mathrm{C}}^{\mathcal{E}}(\boldsymbol{v})\right)$.

On $\mathfrak{M}_{h}^{\mathcal{D}}$ we consider:

- $q^{I}$, the mean-valued interpolation of the integrable field $q$, given by

$$
q^{I}=\left\{\left(\mathbb{P}_{\mathrm{M}}^{\mathrm{D}}(q)\right)_{\mathrm{D} \in \mathfrak{M}_{h}^{\mathcal{D}}}\right\} \text { where } \forall \mathrm{D} \in \mathfrak{M}_{h}^{\mathcal{D}}: \mathbb{P}_{\mathrm{M}}^{\mathrm{D}}(q):=\frac{1}{m_{\mathrm{D}}} \int_{\mathrm{D}} q(\boldsymbol{x}) d V .
$$


In the theoretical analysis, we will consider the mean-valued interpolation on $\mathfrak{M}_{h}^{\mathcal{D}}$ of tensor fields. The extension of definition (37) to tensor fields is carried out component-wisely, and, is hence straightforward. For instance, $\psi^{I}=\left\{\psi_{\mathrm{D}}^{I}\right\}_{\mathrm{D} \in \mathfrak{M}_{h}^{\mathcal{D}}}$ is the mean-valued interpolation of the tensor field $\psi \in \mathfrak{R}^{3 \times 3}$, where for any cell $\mathrm{D}$ of any diamond mesh $\mathfrak{M}_{h}^{\mathcal{D}}$, we let

$$
\left.\psi_{\mathrm{D}}^{I}\right|_{i j}=\frac{1}{m_{\mathrm{D}}} \int_{\mathrm{D}} \psi_{i j}(\boldsymbol{x}) d V
$$

denote the cell average of the $i j$-th component $\psi_{i j}$ over D.

Remark 2 We use the same notation with the superscript I, i.e., $v^{I}$, to denote the mean-valued interpolations of an integrable function $v$ on all the meshes of the mesh family $\left\{\left(\mathfrak{M}_{h}^{\mathcal{T}}, \mathfrak{M}_{h}^{\mathcal{D}}\right)_{h}\right\}$. There is no ambiguity in this choice as it is always possible to deduce which definition is actually applied contextually.

\subsection{Scheme formulation}

The DDFV scheme for the numerical approximation of the steady Stokes equations (1)-(3) reads as:

$$
\begin{aligned}
& \text { find } \boldsymbol{u}_{h} \in \mathfrak{T}_{h, 0}^{3} \text { and } p_{h} \in \mathfrak{D}_{h} \text { such that } \\
& \qquad \begin{aligned}
\operatorname{div}_{h}^{\text {int }}\left(-\eta_{h} \mathrm{D}_{h}\left(\boldsymbol{u}_{h}\right)+p_{h} \mathbb{I}\right) & =\boldsymbol{f}^{I, \text { int }}, \\
\operatorname{div}_{h}^{\mathcal{D}}\left(\boldsymbol{u}_{h}\right)-\lambda h^{2} \Delta_{h}\left(p_{h}\right) & =0, \\
\sum_{\mathrm{D} \in \mathfrak{M}_{h}^{\mathcal{D}}} m_{\mathrm{D}} p_{\mathrm{D}} & =0 .
\end{aligned}
\end{aligned}
$$

where $\boldsymbol{f}^{I, \text { int }}=\left\{\mathbb{P}_{\mathrm{M}}^{\mathcal{P}}(\boldsymbol{f}), \mathbb{P}_{\mathrm{M}}^{\mathcal{V} \text {,int }}(\boldsymbol{f}), \mathbb{P}_{\mathrm{M}}^{\mathcal{E} \text {, int }}\left(\boldsymbol{f}_{h}\right)\right\}$ in equation (39) is the mean-valued interpolation of the loading vector $\boldsymbol{f}$ defined by (31) on the control volumes of $\mathfrak{M}_{h}^{\mathcal{P}}$ and by (32)-(33) restricted to the internal control volumes of $\mathfrak{M}_{h}^{\mathcal{V}}$ and $\mathfrak{M}_{h}^{\mathcal{E F}} ; \eta_{h}$ is a first-order accurate approximation of viscosity $\eta$ satisfying (29); $\lambda$ is the positive stabilization coefficient.

We integrate the momentum conservation law (2) on the primary mesh $\mathfrak{M}_{h}^{\mathcal{P}}$, on the interior node mesh $\mathfrak{M}_{h}^{\mathcal{V}}$, and on the interior edge-face mesh $\mathfrak{M}_{h}^{\mathcal{E} \mathcal{F}}$. Equation (39) can be split into three interconnected sets of equations for the meshes forming $\mathfrak{M}_{h}^{\mathcal{T}}$, i.e., $\mathfrak{M}_{h}^{\mathcal{P}}, \mathfrak{M}_{h}^{\mathcal{V}}$, and $\mathfrak{M}_{h}^{\mathcal{E F}}$, thus giving:

$$
\begin{aligned}
\operatorname{div}_{h}^{\mathcal{P}}\left(-\eta_{h} \mathrm{D}_{h}\left(\boldsymbol{u}_{h}\right)+p_{h} \mathbb{I}\right) & =\mathbb{P}_{\mathrm{M}}^{\mathcal{P}}(\boldsymbol{f}), \\
\operatorname{div}_{h}^{\mathcal{V}, \text { int }}\left(-\eta_{h} \mathrm{D}_{h}\left(\boldsymbol{u}_{h}\right)+p_{h} \mathbb{I}\right) & =\mathbb{P}_{\mathrm{M}}^{\mathcal{V}, \text { int }}(\boldsymbol{f}), \\
\operatorname{div}_{h}^{\mathcal{E} \mathcal{F}, \text { int }}\left(-\eta_{h} \mathrm{D}_{h}\left(\boldsymbol{u}_{h}\right)+p_{h} \mathbb{I}\right) & =\mathbb{P}_{\mathrm{M}}^{\mathcal{E} \mathcal{F}, \text { int }}(\boldsymbol{f}) .
\end{aligned}
$$

The mass conservation equation is directly approached on the diamond mesh using a stabilized term $\grave{a} l a$ Brezzi-Pitkaranta [15] by using the discrete Laplacian given by (28). Equation (40) takes into account the free-divergence constraint (2) and introduces into the scheme the stabilization term. Equation (41) is the discrete version of the additional compatibility condition (6), and is required to ensure the uniqueness of the numerical solution. In fact, as it occurs in the continuous setting, also in the discrete setting the pressure field solving scheme's equations (39) and (40) is defined up to constant scalar fields. To see this, we note that the definition of the discrete divergence in (20) implies that $\operatorname{div}_{h}\left(p_{h} \mathbb{I}\right)=\operatorname{div}_{h}\left(\left(p_{h}+c_{h}\right) \mathbb{I}\right)$ for any constant scalar field $c_{h}=\{c\} \in \mathfrak{D}_{h}$, with $c$ being any real number. Likewise, the definition of the discrete Laplacian in (28) implies that $\Delta_{h}\left(p_{h}\right)=\Delta_{h}\left(p_{h}+c_{h}\right)$. 


\section{Preliminary results}

In this section, we give the results that we will use to analyse the DDFV method. In subsection 4.1, we introduce several concepts, e.g., inner products, mesh dependent norms, discrete duality relations and similar "mimetic" relations. In subsection 4.2, we give a lemma that will be useful in the proof of the Korn inequality, cf. Lemma (8). In subsection 4.3, we present other theoretical tools, as the Poincaré and Korn inequalities, and the error estimates for the interpolation operators of subsection 3.2.

\subsection{Discrete duality relations}

\section{Inner products and mesh-dependent norms}

Let us introduce the following bilinear forms for the elements of the linear spaces $\mathfrak{D}_{h}, \mathfrak{D}_{h}^{3}$ and $\mathfrak{D}_{h}^{3 \times 3}$ :

$$
\begin{aligned}
\forall p, q \in \mathfrak{D}_{h}:[p, q]_{\mathfrak{D}_{h}} & =\sum_{\mathrm{D} \in \mathfrak{M}_{h}^{\mathcal{D}}} m_{\mathrm{D}} p_{\mathrm{D}} q_{\mathrm{D}}, \\
\forall \boldsymbol{u}, \boldsymbol{v} \in \mathfrak{D}_{h}^{3}:[\boldsymbol{u}, \boldsymbol{v}]_{\mathfrak{D}_{h}} & =\sum_{\mathrm{D} \in \mathfrak{M}_{h}^{\mathcal{D}}} m_{\mathrm{D}} \boldsymbol{u}_{\mathrm{D}} \cdot \boldsymbol{v}_{\mathrm{D}}, \\
\forall \phi, \psi \in \mathfrak{D}_{h}^{3 \times 3}:[\phi, \psi]_{\mathfrak{D}_{h}} & =\sum_{\mathrm{D} \in \mathfrak{M}_{h}^{\mathcal{D}}} m_{\mathrm{D}} \phi_{\mathrm{D}}: \psi_{\mathrm{D}}=\sum_{\mathrm{D} \in \mathfrak{M}_{h}^{\mathcal{D}}} m_{\mathrm{D}} \operatorname{Tr}\left(\phi_{\mathrm{D}}^{T} \psi_{\mathrm{D}}\right),
\end{aligned}
$$

(recall that $\left.\phi: \psi=\operatorname{Tr}\left(\phi^{T} \psi\right)\right)$.

Lemma 1 There holds that:

$$
\forall q \in \mathfrak{D}_{h}, \forall \psi \in \mathfrak{D}_{h}^{3 \times 3}:[q, \operatorname{Tr}(\psi)]_{\mathfrak{D}_{h}}=[q \mathbb{I}, \psi]_{\mathfrak{D}_{h}} .
$$

The bilinear forms (45), (46) and (47) are inner products in $\mathfrak{D}_{h}, \mathfrak{D}_{h}^{3}$, and $\mathfrak{D}_{h}^{3 \times 3}$, respectively. These inner products induce the following three mesh-dependent norms:

$$
\begin{array}{rlrl}
\forall q \in \mathfrak{D}_{h}:\|q\|_{\mathfrak{D}_{h}}^{2} & =[q, q]_{\mathfrak{D}_{h}} & & {[\text { using definition (45)], }} \\
\forall \boldsymbol{v} \in \mathfrak{D}_{h}^{3}:\|\boldsymbol{v}\|_{\mathfrak{D}_{h}}^{2}=[\boldsymbol{v}, \boldsymbol{v}]_{\mathfrak{D}_{h}} & & {[\text { using definition (46)], }} \\
\forall \psi \in \mathfrak{D}_{h}^{3 \times 3}:\|\psi \mid\| \|_{\mathfrak{D}_{h}}^{2}=[\psi, \psi]_{\mathfrak{D}_{h}} & & {[\text { using definition (47)]. }}
\end{array}
$$

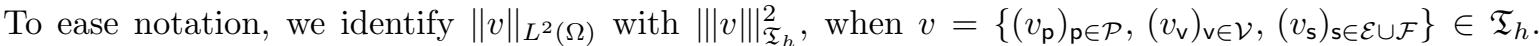
The $L^{2}$-norm for piecewise constant vector and tensor fields is defined by extending component-wisely this definition. Sobolev spaces and corresponding norms for vector and tensor fields are to be intended component-wisely.

Let us introduce the following inner products for the elements of the linear spaces $\mathfrak{T}_{h}$ and $\mathfrak{T}_{h}^{3}$

$$
\begin{aligned}
& \forall u, v \in \mathfrak{T}_{h}:[u, v]_{\mathfrak{T}_{h}}=\frac{1}{3}\left(\sum_{\mathrm{p} \in \mathfrak{M}_{h}^{\mathcal{P}}} m_{\mathrm{p}} u_{\mathrm{p}} v_{\mathrm{p}}+\sum_{\mathrm{v} \in \mathfrak{M}_{h}^{\mathcal{V}}} m_{\mathrm{v}} u_{\mathrm{v}} v_{\mathrm{v}}+\sum_{\mathrm{s} \in \mathfrak{M}_{h}^{\mathcal{E}}} m_{\mathrm{s}} u_{\mathrm{s}} v_{\mathrm{s}}\right), \\
& \forall \boldsymbol{u}, \boldsymbol{v} \in \mathfrak{T}_{h}^{3}:[\boldsymbol{u}, \boldsymbol{v}]_{\mathfrak{T}_{h}}=\frac{1}{3}\left(\sum_{\mathrm{p} \in \mathfrak{M}_{h}^{\mathcal{P}}} m_{\mathrm{p}} \boldsymbol{u}_{\mathrm{p}} \cdot \boldsymbol{v}_{\mathrm{p}}+\sum_{\mathrm{v} \in \mathfrak{M}_{h}^{\mathcal{V}}} m_{\mathrm{v}} \boldsymbol{u}_{\mathrm{v}} \cdot \boldsymbol{v}_{\mathrm{v}}+\sum_{\mathbf{s} \in \mathfrak{M}_{h}^{\mathcal{E F}}} m_{\mathrm{s}} \boldsymbol{u}_{\mathrm{s}} \cdot \boldsymbol{v}_{\mathrm{s}}\right) .
\end{aligned}
$$

These inner products induce the two mesh-dependent norms:

$$
\begin{aligned}
& \forall q \in \mathfrak{T}_{h}:\|\mid\| q \|_{\mathfrak{T}_{h}}^{2}=[q, q]_{\mathfrak{T}_{h}} \quad \text { [using definition (52)], } \\
& \forall \boldsymbol{v} \in \mathfrak{T}_{h}^{3}: \mid\|\boldsymbol{v}\| \|_{\mathfrak{T}_{h}}^{2}=[\boldsymbol{v}, \boldsymbol{v}]_{\mathfrak{T}_{h}} \quad \text { [using definition (53)]. }
\end{aligned}
$$


Moreover, from (30) and in view of inner product definitions (47) and (51), it is straightforward to obtain the inequalities

$$
\forall \psi, \phi \in \mathfrak{D}_{h}^{3 \times 3}: \underline{C}_{\eta}\left|[\psi, \phi]_{\mathfrak{D}_{h}}\right| \leq\left|\left[\eta_{h} \psi, \phi\right]_{\mathfrak{D}_{h}}\right| \leq \bar{C}_{\eta}\left|[\psi, \phi]_{\mathfrak{D}_{h}}\right| .
$$

The three discrete duality relations established in the following lemma, i.e., (57), (58), and (59), are discrete versions of integration by parts formulas that hold for the discrete divergence and gradient operators introduced in subsection 3.2, assuming that these latters act on grid functions defined on $\mathfrak{M}_{h}^{\mathcal{T}}$ that are zero on the boundary.

\section{Lemma 2 (Discrete duality relations)}

(i) The first discrete duality relation is given by

$$
\forall \boldsymbol{v} \in \mathfrak{D}_{h}^{3}, \forall q \in \mathfrak{T}_{h, 0}:\left[\operatorname{div}_{h}(\boldsymbol{v}), q\right]_{\mathfrak{T}_{h}}+\left[\boldsymbol{v}, \nabla_{h} q\right]_{\mathfrak{D}_{h}}=0,
$$

(ii) the second discrete duality relation is given by

$$
\forall \phi \in \mathfrak{D}_{h}^{3 \times 3}, \forall \boldsymbol{v} \in \mathfrak{T}_{h, 0}^{3}:\left[\operatorname{div}_{h}(\phi), \boldsymbol{v}\right]_{\mathfrak{T}_{h}}+\left[\phi, \nabla_{h} \boldsymbol{v}\right]_{\mathfrak{D}_{h}}=0,
$$

(iii) the third discrete duality relation is given by

$$
\forall q \in \mathfrak{D}_{h}, \forall \boldsymbol{v} \in \mathfrak{T}_{h, 0}^{3}:\left[\operatorname{div}_{h}(q \mathbb{I}), \boldsymbol{v}\right]_{\mathfrak{T}_{h}}+\left[q, \operatorname{Tr}\left(\nabla_{h} \boldsymbol{v}\right)\right]_{\mathfrak{D}_{h}}=0 .
$$

\section{A mesh-dependent seminorm}

For the elements of the linear space $\mathfrak{D}_{h}$, we make use of the seminorm $|\cdot|_{h}$ given by

$$
|q|_{h}^{2}=\sum_{\left(\mathrm{D} \mid \mathrm{D}^{\prime}\right)} \frac{h_{\mathrm{D}}+h_{\mathrm{D}^{\prime}}}{2}\left|q_{\mathrm{D}}-q_{\mathrm{D}^{\prime}}\right|^{2},
$$

(recall that $\left(D \mid D^{\prime}\right)$ denote the pairs $\left(D, D^{\prime}\right) \in \mathfrak{M}_{h}^{\mathcal{D}} \times \mathfrak{M}_{h}^{\mathcal{D}}$ such that the surface $\overline{\mathrm{D}} \cap \overline{\mathrm{D}^{\prime}}$ has a non-zero two-dimensional measure). Using this seminorm definition, the discrete Laplacian given in (61) and the inner product introduced in (45) for the scalar fields of $\mathfrak{D}_{h}$ make it possible to obtain a discrete analog of the exact relation:

$$
\forall q \in H^{2}(\Omega) \cap H_{0}^{1}(\Omega): \int_{\Omega}|\nabla q|^{2} d V+\int_{\Omega} q \Delta(q) d V=0 .
$$

This result, which will be used in the analysis of the next subsections, is stated in the lemma below.

Lemma 3 There holds that

$$
\forall q \in \mathfrak{D}_{h}:|q|_{h}^{2}+\left[q, \Delta_{h} q\right]_{\mathfrak{D}_{h}}=0
$$

\subsection{First property needed in the proof of the Korn inequality}

We give with a lemma that will be useful in the proof of the Korn inequality, cf. Lemma (8). From calculus, we know that the identity below holds for any smooth vector field $v$ :

$$
\operatorname{div}\left((\nabla \boldsymbol{v})^{T}\right)=\operatorname{div}(\operatorname{div}(\boldsymbol{v}) \mathbb{I}) .
$$

A discrete analog is stated as follows.

Lemma 4 There holds that

$$
\forall \boldsymbol{v} \in \mathfrak{T}_{h, 0}^{3}: \quad \operatorname{div}_{h}\left(\nabla_{h} \boldsymbol{v}\right)^{T}=\operatorname{div}_{h}\left(\operatorname{div}_{h}^{\mathcal{D}}(\boldsymbol{v}) \mathbb{I}\right) .
$$


Proof .To ease notation, let

$$
\psi=\left(\nabla_{h} \boldsymbol{v}\right)^{T}-\operatorname{div}_{h}^{\mathcal{D}}(\boldsymbol{v}) \mathbb{I} \in \mathfrak{D}_{h}^{3 \times 3} .
$$

We will show that $\operatorname{div}_{h} \psi=0$. Let us denote the three spatial components of vector $\boldsymbol{v} \in \mathfrak{T}_{h}^{3}$ by $v_{i}$ for $i=1,2,3$, its value at points A, B etc by the vector symbols $\boldsymbol{v}_{\mathrm{A}}, \boldsymbol{v}_{\mathrm{B}}$ etc, and the $i$-th canonical basis vector of $\mathfrak{R}^{3}$ by $\mathbf{e}_{i}$. A direct calculation gives the explicit form of $\psi_{\mid \mathrm{D}}$ the restriction of $\psi$ to the diamond cell D:

$$
\psi_{\mid \mathrm{D}}=\left(\begin{array}{ccc}
-\sum_{i=2}^{3} \nabla_{h}^{\mathrm{D}} v_{i} \cdot \mathbf{e}_{i} & \nabla_{h}^{\mathrm{D}} v_{2} \cdot \mathbf{e}_{1} & \nabla_{h}^{\mathrm{D}} v_{3} \cdot \mathbf{e}_{1} \\
\nabla_{h}^{\mathrm{D}} v_{1} \cdot \mathbf{e}_{3} & -\sum_{i=1, i \neq 2}^{3} \nabla_{h}^{\mathrm{D}} v_{i} \cdot \mathbf{e}_{i} & \nabla_{h}^{\mathrm{D}} v_{3} \cdot \mathbf{e}_{2} \\
\nabla_{h}^{\mathrm{D}} v_{1} \cdot \mathbf{e}_{3} & \nabla_{h}^{\mathrm{D}} v_{2} \cdot \mathbf{e}_{3} & -\sum_{i=1}^{2} \nabla_{h}^{\mathrm{D}} v_{i} \cdot \mathbf{e}_{i}
\end{array}\right)
$$

Using this expression, we easily obtain for the vector $\mathbf{N}_{\mathrm{K}, \mathrm{L}}=\left(\mathbf{N}_{\mathrm{K}, \mathrm{L}} \cdot \mathbf{e}_{1}, \mathbf{N}_{\mathrm{K}, \mathrm{L}} \cdot \mathbf{e}_{2}, \mathbf{N}_{\mathrm{K}, \mathrm{L}} \cdot \mathbf{e}_{3}\right)^{T}$ that

$$
\psi_{\mid \mathrm{D}} \mathbf{N}_{\mathrm{K}, \mathrm{L}}=\left(\begin{array}{c}
-\sum_{i=2}^{3} \nabla_{h}^{\mathrm{D}} v_{i} \cdot \mathbf{e}_{i} \mathbf{N}_{\mathrm{K}, \mathrm{L}} \cdot \mathbf{e}_{1}+\nabla_{h}^{\mathrm{D}} v_{2} \cdot \mathbf{e}_{1} \mathbf{N}_{\mathrm{K}, \mathrm{L}} \cdot \mathbf{e}_{2}+\nabla_{h}^{\mathrm{D}} v_{3} \cdot \mathbf{e}_{1} \mathbf{N}_{\mathrm{K}, \mathrm{L}} \cdot \mathbf{e}_{3} \\
\nabla_{h}^{\mathrm{D}} v_{1} \cdot \mathbf{e}_{3} \mathbf{N}_{\mathrm{K}, \mathrm{L}} \cdot \mathbf{e}_{1}-\sum_{i=1, i \neq 2}^{3} \nabla_{h}^{\mathrm{D}} v_{i} \cdot \mathbf{e}_{i} \mathbf{N}_{\mathrm{K}, \mathrm{L}} \cdot \mathbf{e}_{2}+\nabla_{h}^{\mathrm{D}} v_{3} \cdot \mathbf{e}_{2} \mathbf{N}_{\mathrm{K}, \mathrm{L}} \cdot \mathbf{e}_{3} \\
\nabla_{h}^{\mathrm{D}} v_{1} \cdot \mathbf{e}_{3} \mathbf{N}_{\mathrm{K}, \mathrm{L}} \cdot \mathbf{e}_{1}+\nabla_{h}^{\mathrm{D}} v_{2} \cdot \mathbf{e}_{3} \mathbf{N}_{\mathrm{K}, \mathrm{L}} \cdot \mathbf{e}_{2}-\sum_{i=1}^{2} \nabla_{h}^{\mathrm{D}} v_{i} \cdot \mathbf{e}_{i} \mathbf{N}_{\mathrm{K}, \mathrm{L}} \cdot \mathbf{e}_{3}
\end{array}\right) .
$$

After some algebraic manipulations, we can rewrite the previous expression in the compact form that involves two vector products:

$$
\psi_{\mathrm{I}} \mathbf{N}_{\mathrm{K}, \mathrm{L}}=\frac{\boldsymbol{v}_{\mathrm{A}}-\boldsymbol{v}_{\mathrm{B}}}{2} \times\left(\boldsymbol{x}_{\mathrm{F}}-\boldsymbol{x}_{\mathrm{E}}\right)+\frac{\boldsymbol{v}_{\mathrm{F}}-\boldsymbol{v}_{\mathrm{E}}}{2} \times\left(\boldsymbol{x}_{\mathrm{B}}-\boldsymbol{x}_{\mathrm{A}}\right) .
$$

Likewise, we deduce that

$$
\begin{aligned}
& \psi_{\mid \mathrm{D}} \mathbf{N}_{\mathrm{A}, \mathrm{B}}=\frac{\boldsymbol{v}_{\mathrm{L}}-\boldsymbol{v}_{\mathrm{K}}}{2} \times\left(\boldsymbol{x}_{\mathrm{F}}-\boldsymbol{x}_{\mathrm{E}}\right)+\frac{\boldsymbol{v}_{\mathrm{E}}-\boldsymbol{v}_{\mathrm{F}}}{2} \times\left(\boldsymbol{x}_{\mathrm{L}}-\boldsymbol{x}_{\mathrm{K}}\right), \\
& \psi_{\mid \mathrm{D}} \mathbf{N}_{\mathrm{E}, \mathrm{F}}=\frac{\boldsymbol{v}_{\mathrm{K}}-\boldsymbol{v}_{\mathrm{L}}}{2} \times\left(\boldsymbol{x}_{\mathrm{B}}-\boldsymbol{x}_{\mathrm{A}}\right)+\frac{\boldsymbol{v}_{\mathrm{B}}-\boldsymbol{v}_{\mathrm{A}}}{2} \times\left(\boldsymbol{x}_{\mathrm{L}}-\boldsymbol{x}_{\mathrm{K}}\right) .
\end{aligned}
$$

In accordance with the definition of the divergence operator given in (20), we have that

$$
\operatorname{div}_{h} \psi=\left(\left(\operatorname{div}_{h}^{\mathrm{p}}(\psi)\right)_{\mathbf{p} \in \mathfrak{M}_{h}^{\mathcal{P}}},\left(\operatorname{div}_{h}^{\mathrm{v}}(\psi)\right)_{\mathbf{v} \in \mathfrak{M}_{h}^{\nu}},\left(\operatorname{div}_{h}^{\mathrm{s}}(\psi)\right)_{\mathbf{s} \in \mathfrak{M}_{h}^{\mathcal{E F}}}\right) .
$$

For simplicity of notation, in the next formulas we will implicitly refer the six points A, B, E, F, $\mathrm{K}$, L to each diamond cell determined by the summation index $\mathrm{D}$, and we will properly adjust the orientation of the normal vectors $\mathbf{N}_{\mathrm{D}, \mathrm{p}}, \mathbf{N}_{\mathrm{D}, \mathrm{v}}$, and $\mathbf{N}_{\mathrm{D}, \mathrm{s}}$ that appears in (13) to have a positive sign. Accordingly, the three components of $\operatorname{div}_{h} \psi$ in (68) are given by

$$
\begin{aligned}
& m_{\mathrm{p}} \operatorname{div}_{h}^{\mathrm{p}}(\psi)=\sum_{\mathrm{D} \in \mathfrak{M}_{h \mid \mathrm{p}}^{\mathcal{D}}}\left(\frac{\boldsymbol{v}_{\mathrm{A}}-\boldsymbol{v}_{\mathrm{B}}}{2} \times\left(\boldsymbol{x}_{\mathrm{F}}-\boldsymbol{x}_{\mathrm{E}}\right)+\frac{\boldsymbol{v}_{\mathrm{F}}-\boldsymbol{v}_{\mathrm{E}}}{2} \times\left(\boldsymbol{x}_{\mathrm{B}}-\boldsymbol{x}_{\mathrm{A}}\right)\right), \\
& m_{\mathrm{v}} \operatorname{div}_{h}^{\mathrm{v}}(\psi)=\sum_{\mathrm{D} \in \mathfrak{M}_{h \mid \mathrm{v}}^{\mathcal{D}}}\left(\frac{\boldsymbol{v}_{\mathrm{L}}-\boldsymbol{v}_{\mathrm{K}}}{2} \times\left(\boldsymbol{x}_{\mathrm{F}}-\boldsymbol{x}_{\mathrm{E}}\right)+\frac{\boldsymbol{v}_{\mathrm{E}}-\boldsymbol{v}_{\mathrm{F}}}{2} \times\left(\boldsymbol{x}_{\mathrm{L}}-\boldsymbol{x}_{\mathrm{K}}\right)\right), \\
& m_{\mathbf{s}} \operatorname{div}_{h}^{\mathbf{s}}(\psi)=\sum_{\mathrm{D} \in \mathfrak{M}_{h}^{\mathcal{D}} \mathfrak{s}}\left(\frac{\boldsymbol{v}_{\mathrm{K}}-\boldsymbol{v}_{\mathrm{L}}}{2} \times\left(\boldsymbol{x}_{\mathrm{B}}-\boldsymbol{x}_{\mathrm{A}}\right)+\frac{\boldsymbol{v}_{\mathrm{B}}-\boldsymbol{v}_{\mathrm{A}}}{2} \times\left(\boldsymbol{x}_{\mathrm{L}}-\boldsymbol{x}_{\mathrm{K}}\right)\right) .
\end{aligned}
$$

Eventually, identity (63) is a consequence of Proposition A.1, which is reported in the final appendix. 


\subsection{Technical lemmas}

In this section, we present several results on the discrete operators. We review the results of [18] by adapted them to the vector-valued setting and we review the results of [33] by extending them to the 3 -D case. We only prove results which are specific to the three dimensions and to the vector-case.

Lemma 5 (Poincaré inequality) Let $\mathfrak{M}_{h}^{\mathcal{T}}$ be a mesh triplet for the domain $\Omega$. Then, there exists a positive constant $C_{1}$, which is independent of $h$ and only depends on the diameter of $\Omega$ and the regularity constant $C_{\text {reg }}$, such that

$$
\forall \boldsymbol{v} \in \mathfrak{T}_{h, 0}^{3}:\left.\quad\|\boldsymbol{v}\|\right|_{\mathfrak{T}_{h}} \leq C_{1}\left\|\mid \nabla_{h} \boldsymbol{v}\right\|_{\mathfrak{D}_{h}} .
$$

Lemma 6 There exists a real positive constant $C_{2}$ independent of $h$ such that for every $\boldsymbol{v} \in\left(H^{2}(\Omega)\right)^{3}$ there holds that:

$$
\left\|\boldsymbol{v}-\boldsymbol{v}^{J}\right\|_{L^{2}(\Omega)}+\left\|\nabla \boldsymbol{v}-\nabla_{h} \boldsymbol{v}^{J}\right\|_{L^{2}(\Omega)} \leq C_{2} h\|\boldsymbol{v}\|_{H^{2}(\Omega)} .
$$

Lemma 7 There exists a constant $C_{3}$ independent of $h$ such that for every $q \in H^{1}(\Omega)$ and for any cell $D$ of any diamond mesh $\mathfrak{M}_{h}^{\mathcal{D}}$ there holds:

$$
\left\|q^{I}-q\right\|_{L^{2}(D)} \leq C_{3} h_{D}\|q\|_{H^{1}(D)}
$$

Lemma 8 (Discrete Korn inequality) For every $\boldsymbol{v} \in \mathfrak{T}_{h, 0}^{3}$ there holds

$$
\left.\left\|\left|D_{h}(\boldsymbol{v})\right|\right\|\right|_{\mathfrak{D}_{h}} \leq\left\|\left|\nabla_{h} \boldsymbol{v}\left\|_{\mathfrak{D}_{h}} \leq \sqrt{2}\right\|\right| D_{h}(\boldsymbol{v}) \mid\right\|_{\mathfrak{D}_{h}} .
$$

We will refer to the right-most inequality as the discrete Korn inequality.

Proof. The left inequality in (72) is obviously true because the norm of the symmetric part of a matrix, cf. (27), is always controlled by the norm of the full matrix.

To prove the right inequality in (72), we first note that a straightforward calculation using definition (27) for the discrete strain rate tensor yields:

$$
\left\|\mid \mathrm{D}_{h}(\boldsymbol{v})\right\|_{\mathfrak{D}_{h}}^{2}=\frac{1}{2}\left(\left\|\nabla_{h} \boldsymbol{v}\right\|_{\mathfrak{D}_{h}}^{2}+\left[\nabla_{h} \boldsymbol{v},\left(\nabla_{h} \boldsymbol{v}\right)^{T}\right]_{\mathfrak{D}_{h}}\right) .
$$

We will prove the discrete Korn inequality by showing that the second term in the right-hand side of identity (73) is positive. To this purpose, we begin from the second discrete duality relation given by (58) to obtain:

$$
\begin{array}{rlrl}
{\left[\nabla_{h} \boldsymbol{v},\left(\nabla_{h} \boldsymbol{v}\right)^{T}\right]_{\mathfrak{D}_{h}}} & =-\left[\boldsymbol{v}, \operatorname{div}_{h}\left(\nabla_{h} \boldsymbol{v}\right)^{T}\right]_{\mathfrak{T}_{h}} & & {[\text { use Lemma 4, inequality (63)] }} \\
& =-\left[\boldsymbol{v}, \operatorname{div}_{h}\left(\operatorname{div}_{h}^{\mathcal{D}}(\boldsymbol{v}) \mathbb{I}\right)\right]_{\mathfrak{T}_{h}} & & \text { [use third discrete duality relation (59)] } \\
& =\left[\operatorname{Tr}\left(\nabla_{h} \boldsymbol{v}\right), \operatorname{div}_{h}^{\mathcal{D}}(\boldsymbol{v})\right]_{\mathfrak{D}_{h}} & & \text { [use definition (26) and norm (50)] } \\
& =\|\| \operatorname{div}_{h}^{\mathrm{D}}(\boldsymbol{v}) \|_{\mathfrak{D}_{h}}^{2}
\end{array}
$$

Lemma 9 There exists a real positive constant $C_{4}$ independent of $h$ such that for every $q \in H^{1}(\Omega)$ there holds that:

$$
\left|q^{I}\right|_{h} \leq C_{4}|q|_{H^{1}(\Omega)}
$$


Proof. Let $q^{I}=\left\{\left(q_{\mathrm{D}}^{I}\right)_{\mathrm{D}}\right\} \in \mathfrak{D}_{h}$ be the mean-valued $\mathfrak{D}_{h}$-interpolation of a generic function $q \in H^{1}(\Omega)$ given by (37), where $q_{\mathrm{D}}^{I}=\left.q^{I}\right|_{\mathrm{D}}$. For every face $\sigma \in \partial \mathrm{D}$ of every diamond cell $\mathrm{D} \in \mathfrak{M}_{h}^{\mathcal{D}}$, applying Jensen inequality yields:

$$
\left|q_{\mathrm{D}}^{I}-\frac{1}{|\sigma|} \int_{\sigma} q d S\right|^{2} \leq \frac{1}{|\sigma|} \int_{\sigma}\left|q_{\mathrm{D}}^{I}-q\right|^{2} d S=\frac{1}{|\sigma|}\left\|q_{\mathrm{D}}^{I}-q\right\|_{L^{2}(\sigma)}^{2} .
$$

Using Agmon inequality, noting that the $H^{1}(\mathrm{D})$-seminorm of $q_{\mathrm{D}}^{I}$ is zero because $q_{\mathrm{D}}^{I}$ is constant on $\mathrm{D}$, applying the estimate for the interpolation error on the diamond cell $\mathrm{D}$ provided by Lemma 7 , and using the scaling property $C_{r e g}^{2} h_{\mathrm{D}}^{2} \leq|\sigma|$ that holds for every $\sigma \in \partial \mathrm{D}$ allows us to obtain the following chain of inequalities

$$
\frac{1}{|\sigma|}\left\|q_{\mathrm{D}}^{I}-q\right\|_{L^{2}(\sigma)}^{2} \leq \frac{C_{A g}}{|\sigma|}\left(h_{\mathrm{D}}^{-1}\left\|q_{\mathrm{D}}^{I}-q\right\|_{L^{2}(\mathrm{D})}^{2}+h_{\mathrm{D}}|q|_{H^{1}(\mathrm{D})}^{2}\right) \leq \frac{C_{A g}\left(1+C_{3}^{2}\right)}{C_{r e g}^{2}} h_{\mathrm{D}}^{-1}|q|_{H^{1}(\mathrm{D})}^{2} .
$$

Substituting (77) into (76) readily gives:

$$
\forall \sigma \in \partial \mathrm{D}, \forall \mathrm{D} \in \mathfrak{M}_{h}^{\mathcal{D}}:\left|q_{\mathrm{D}}^{I}-\frac{1}{|\sigma|} \int_{\sigma} q d S\right|^{2} \leq \frac{C_{A g}\left(1+C_{3}^{2}\right)}{C_{r e g}^{2}} h_{\mathrm{D}}^{-1}|q|_{H^{1}(\mathrm{D})}^{2} .
$$

Now, let us observe that adding and subtracting the face average $q_{\sigma}=\frac{1}{|\sigma|} \int_{\sigma} q d S$, noting that $\left|q_{\mathrm{D}}^{I}-q_{\mathrm{D}^{\prime}}^{I}\right|^{2} \leq 2\left|q_{\mathrm{D}^{\prime}}^{I}-q_{\sigma}\right|^{2}+2\left|q_{\mathrm{D}}^{I}-q_{\sigma}\right|^{2}$, using inequality (78), and the scaling property $\max \left(h_{\mathrm{D}} /\right.$ $\left.h_{\mathrm{D}^{\prime}}, h_{\mathrm{D}^{\prime}} / h_{\mathrm{D}}\right) \leq 1 / C_{\text {reg }}$ which follows from $\left(\mathrm{M}_{2}\right)$ yield:

$$
\left|q^{I}\right|_{h}^{2} \leq \sum_{\left(\mathrm{D} \mid \mathrm{D}^{\prime}\right)}\left(h_{\mathrm{D}}+h_{\mathrm{D}^{\prime}}\right)\left(\left|q_{\mathrm{D}}^{I}-q_{\sigma}\right|^{2}+\left|q_{\mathrm{D}^{\prime}}^{I}-q_{\sigma}\right|^{2}\right) \leq C_{4}^{2} \sum_{\mathrm{D} \in \mathfrak{M}_{h}^{\mathcal{D}}}|q|_{H^{1}(\mathrm{D})}^{2}=C_{4}^{2}|q|_{H^{1}(\Omega)}^{2},
$$

where $C_{4}^{2}=4 C_{A g}\left(1+1 / C_{r e g}\right)\left(1+C_{3}^{2}\right) / C_{r e g}^{2}$.

Lemma 10 There exists a real positive constant $C_{5}$ independent of $h$ such that:

$$
\begin{aligned}
& \forall \boldsymbol{v} \in\left(H^{1}(\Omega)\right)^{3}:\|\| \nabla_{h} \boldsymbol{v}^{I}\left\|_{\mathfrak{D}_{h}} \leq C_{5}\right\| \boldsymbol{v} \|_{H^{1}(\Omega)}, \\
& \forall \boldsymbol{v} \in\left(H^{2}(\Omega)\right)^{3},:\left\|\nabla_{h} \boldsymbol{v}^{J}\right\|_{\mathfrak{D}_{h}} \leq C_{5}\|\boldsymbol{v}\|_{H^{2}(\Omega)} .
\end{aligned}
$$

Proof. To ease notation, let $v^{I}$ be one of the three spatial components $v_{i}^{I}$ for $i=1,2,3$ of the interpolation vector $\boldsymbol{v}^{I}=\left(v_{1}^{I}, v_{2}^{I}, v_{3}^{I}\right)^{T}$, and note that

$$
\left|\nabla_{h}^{\mathrm{D}} v^{I}\right|^{2} \leq 3\left(\frac{1}{3 m_{\mathrm{D}}}\right)^{2}\left(\left|v_{\mathrm{L}}^{I}-v_{\mathrm{K}}^{I}\right|^{2}\left|\mathbf{N}_{\mathrm{K}, \mathrm{L}}\right|^{2}+\left|v_{\mathrm{B}}^{I}-v_{\mathrm{A}}^{I}\right|^{2}\left|\mathbf{N}_{\mathrm{A}, \mathrm{B}}\right|^{2}+\left|v_{\mathrm{F}}^{I}-v_{\mathrm{E}}^{I}\right|^{2}\left|\mathbf{N}_{\mathrm{E}, \mathrm{F}}\right|^{2}\right),
$$

where $v_{\mathrm{A}}^{I}, v_{\mathrm{B}}^{I}, v_{\mathrm{E}}^{I}, v_{\mathrm{F}}^{I}, v_{\mathrm{K}}^{I}, v_{\mathrm{L}}^{I}$ are the degrees of freedom of $v^{I}$ for the six points $\mathrm{A}, \mathrm{B}, \mathrm{E}, \mathrm{F}, \mathrm{K}, \mathrm{L}$ that we defined for the diamond cell $\mathrm{D}$ in the mesh construction algorithm. Now, let us consider the face $\sigma=\partial \mathrm{p}_{\mathrm{K}} \cap \partial \mathrm{p}_{\mathrm{L}}$ that is shared by the cells $\mathrm{p}_{\mathrm{K}}$ and $\mathrm{p}_{\mathrm{L}}$ related to points $\mathrm{K}$ and $\mathrm{L}$, respectively, and denote the average of $v$ on face $\sigma$ by $v_{\sigma}=\frac{1}{|\sigma|} \int_{\sigma} v d S$. The approximation result of Lemma A.1 in the final appendix implies that

$$
\left|v_{\sigma}-v_{\mathrm{K}}^{I}\right|^{2} \leq C_{18} \frac{\operatorname{diam}\left(\mathrm{p}_{\mathrm{K}}\right)}{|\sigma|} \int_{\mathbf{p}_{\mathrm{K}}}|\nabla v|^{2} d V .
$$

Adding and subtracting $v_{\sigma}$ in the finite difference $\left|v_{\mathrm{L}}^{I}-v_{\mathrm{K}}^{I}\right|$, using the triangular inequality, applying (83), and noting that the scaling properties listed in $\left(\mathrm{M}_{2}\right)$ implies that $\frac{\left|\mathbf{N}_{\mathrm{K}, \mathrm{L}}\right|^{2}}{m_{\mathrm{D}}} \frac{\operatorname{diam}\left(\mathrm{p}_{\mathrm{K}}\right)}{|\sigma|} \leq \frac{1}{C_{r e g}^{4}}$ 
provides us this upper bound:

$$
3 m_{\mathrm{D}} \frac{1}{\left(3 m_{\mathrm{D}}\right)^{2}}\left|v_{\mathrm{L}}^{I}-v_{\mathrm{K}}^{I}\right|^{2}\left|\mathbf{N}_{\mathrm{K}, \mathrm{L}}\right|^{2} \leq \frac{2}{3} \frac{C_{18}}{C_{r e g}^{4}} \int_{\mathrm{p}_{\mathrm{K}} \cup \mathrm{p}_{\mathrm{L}}}|\nabla v|^{2} d V
$$

Two similar inequalities can also be derived for the terms involving the finite differences $\left|v_{\mathrm{B}}^{I}-v_{\mathrm{A}}^{I}\right|$ and $\left|v_{\mathrm{F}}^{I}-v_{\mathrm{E}}^{I}\right|$. Therefore, we deduce that

$$
\left\|\left|\nabla_{h} \boldsymbol{v}^{I}\right|\right\|_{\mathfrak{M}_{h}^{\mathcal{D}}}^{2} \leq \frac{2}{3} \frac{C_{18}}{C_{r e g}^{4}} \sum_{\mathrm{D} \in \mathfrak{M}_{h}^{\mathcal{D}}}\left(\int_{\mathfrak{P}_{\mathrm{K}} \cup p_{\mathrm{L}}}|\nabla v|^{2} d V+\int_{\mathrm{v}_{\mathrm{A}} \cup \cup_{\mathrm{B}}}|\nabla v|^{2} d V+\int_{\mathrm{S}_{\mathrm{E}} \cup \mathrm{S}_{\mathrm{F}}}|\nabla v|^{2} d V\right) \leq \widetilde{\mathcal{N}}_{\overline{3}}^{2} \frac{C_{18}}{C_{r e g}^{4}}\|\nabla v\|_{L^{2}(\Omega)},
$$

where again $\mathrm{v}_{\mathrm{A}}, \mathrm{v}_{\mathrm{B}}, \mathrm{s}_{\mathrm{E}}, \mathrm{s}_{\mathrm{F}}$ are the control volumes related to the points $\mathrm{A}, \mathrm{B}, \mathrm{E}, \mathrm{F}$, and $\widetilde{\mathcal{N}}=2\left(\mathcal{N}_{*}+\right.$ $\left.\mathcal{N}_{\mathcal{V}}+\mathcal{N}_{\mathcal{E F}}\right)$. We recall that $\mathcal{N}_{*}$ is the integer constant provided by consequence $\left(\mathrm{M}_{1}\right)$, while $\mathcal{N}_{\mathcal{V}}$ and $\mathcal{N}_{\mathcal{E} F}$ are the integer constants introduced in Assumption $\left(\mathrm{A}_{2}\right)$. The first statement of this lemma follows by applying the previous inequality to each component of vector $\boldsymbol{v}^{I}$.

The second lemma statement follows by extending to the vector case [33] the similar result for scalar fields proved in [18].

Lemma 11 There exists a real positive constant $C_{6}$ independent of $h$ such that for every $\boldsymbol{v} \in\left(H^{2}(\Omega)\right)^{3}$, with $\operatorname{div}(\boldsymbol{v})=0$ there holds that:

$$
\left\|\operatorname{div}_{h}^{\mathcal{D}}\left(\boldsymbol{v}^{J}\right) \mid\right\|_{\mathfrak{D}_{h}} \leq C_{6} h\|\boldsymbol{v}\|_{H^{2}(\Omega)} .
$$

Proof. Subtracting $\operatorname{div}(\boldsymbol{v})=0$ in the left-hand side of (86), using definition (25) and $\operatorname{div}(\boldsymbol{v})=\operatorname{Tr}(\nabla \boldsymbol{v})$, the fact the norm of a matrix trace is bounded by the norm of the matrix, and the result of Lemma 6 , cf. inequality (70), yields to the result with $C_{6}=C_{2}$.

Lemma 12 There exists a real positive constant $C_{7}$ independent of $h$ such that for every $\boldsymbol{v} \in\left(H^{2}(\Omega)\right)^{3}$, and for every cell $D$ of any diamond mesh $\mathfrak{M}_{h}^{\mathcal{D}}$ there holds:

$$
\left\|D_{h}\left(\boldsymbol{v}^{J}\right)-D(\boldsymbol{v})_{\mid D}^{I}\right\|_{L^{2}(D)} \leq C_{7} h_{D}\|\boldsymbol{v}\|_{H^{2}(D)},
$$

where $D(\boldsymbol{v})^{I} \in \mathfrak{D}_{h}^{3 \times 3}$ is the mean-valued interpolation of $D(\boldsymbol{v})$ on mesh $\mathfrak{M}_{h}^{\mathcal{D}}$ given by (37)-(38).

Proof. Using Jensen inequality, noting that the norm of a symmetric part of a matrix is bounded from above by the norm of the full matrix, and finally applying the result of Lemma 6 , cf. inequality (70), yield to the result with $C_{7}=C_{2}$.

Lemma 13 There exists a constant $C_{8}$ independent of $h$ such that for every $\boldsymbol{v} \in\left(H^{1}(\Omega)\right)^{3}$ and every $q \in \mathfrak{D}_{h}$ there holds:

$$
\sum_{D \in \mathfrak{M}_{h}^{D}} \int_{D} q_{D}\left(\operatorname{div}_{h}^{D}\left(\boldsymbol{v}^{I}\right)-\operatorname{div}(\boldsymbol{v})\right) d V \leq C_{8} h|q|_{h}\|\boldsymbol{v}\|_{H^{1}(\Omega)} .
$$

Proof. Let $\mathcal{T}_{i_{1} i_{2} i_{3}}$ be a triangular face of the boundary $\partial \mathrm{D}$ of the diamond cell $\mathrm{D}$. These faces are given by the eight possible combinations of indices $\left(i_{1}, i_{2}, i_{3}\right)$ where $i_{1} \in\{\mathrm{A}, \mathrm{B}\}, i_{2} \in\{\mathrm{K}, \mathrm{L}\}$, and $i_{3} \in\{\mathrm{E}, \mathrm{F}\}$. Moreover, assume that the orientation of $\mathcal{T}_{i_{1} i_{2} i_{3}}$ is such that the normal vector to the face points out of the diamond cell D. Note that

$$
\operatorname{div}_{h}^{\mathrm{D}}\left(\boldsymbol{v}^{I}\right)=\frac{1}{m_{\mathrm{D}}} \sum_{\sigma \in \partial \mathrm{D}}|\sigma| \boldsymbol{v}_{\sigma}^{I} \cdot \mathbf{n}_{\mathrm{D}, \sigma} \quad \text { where } \quad \boldsymbol{v}_{\sigma}^{I}=\frac{1}{3} \sum_{i=1}^{3} \boldsymbol{v}_{i}^{I},
$$


and $\boldsymbol{v}_{i}^{I}$ for $i \in\left\{i_{1}, i_{2}, i_{3}\right\}$ are the degrees of freedom of face $\sigma=\mathcal{T}_{i_{1} i_{2} i_{3}} \subset \partial \mathrm{D}$. For example, cf. Fig. 1$(a)$, if $\sigma=\mathcal{T}_{\mathrm{AKE}}$, then $\boldsymbol{v}_{\sigma}^{I}=(1 / 3)\left(\boldsymbol{v}_{\mathrm{A}}^{I}+\boldsymbol{v}_{\mathrm{E}}^{I}+\boldsymbol{v}_{\mathrm{K}}^{I}\right)$, and, by comparison, $\boldsymbol{v}_{i_{1}}^{I}=\boldsymbol{v}_{\mathrm{A}}^{I}$, etc. To ease notation, let $\boldsymbol{v}_{\sigma}$ denote the average of $\boldsymbol{v}$ on face $\sigma$

$$
\boldsymbol{v}_{\sigma}=\frac{1}{|\sigma|} \int_{\sigma} \boldsymbol{v} d S
$$

and $\boldsymbol{R}_{\sigma}(\boldsymbol{v})$ the face-based quantity given by:

$$
\boldsymbol{R}_{\sigma}(\boldsymbol{v})=\frac{1}{|\sigma|} \int_{\sigma}\left(\boldsymbol{v}_{\sigma}^{I}-\boldsymbol{v}\right) d S
$$

Using definitions (90) and (91), formula (89) and the divergence theorem make it possible to obtain the development:

$$
\int_{\mathrm{D}}\left(\operatorname{div}_{h}^{\mathrm{D}}\left(\boldsymbol{v}^{I}\right)-\operatorname{div}(\boldsymbol{v})\right) d V=\sum_{\sigma \in \partial \mathrm{D}} \int_{\sigma}\left(\boldsymbol{v}_{\sigma}^{I}-\boldsymbol{v}\right) \cdot \mathbf{n}_{\mathrm{D}, \sigma} d S=\sum_{\sigma \in \partial \mathrm{D}}|\sigma| \mathbf{n}_{\mathrm{D}, \sigma} \cdot \boldsymbol{R}_{\sigma}(\boldsymbol{v}) .
$$

We multiply both sides of identity (92) by $q_{\mathrm{D}}$ and sum on all the diamond cells $\mathrm{D} \in \mathfrak{M}_{h}^{\mathcal{D}}$. Then, we reorder the summation terms on the faces that are shared by adjacent diamond cells, multiply and divide the summation argument by $\left(\left(h_{\mathrm{D}}+h_{\mathrm{D}^{\prime}}\right) / 2\right)^{1 / 2}$ and use the Cauchy-Schwarz inequality. This leads to the following development:

$$
\begin{aligned}
\sum_{\mathrm{D} \in \mathfrak{M}_{h}^{D}} q_{\mathrm{D}} \int_{\mathrm{D}}\left(\operatorname{div}_{h}^{\mathrm{D}}\left(\boldsymbol{v}^{I}\right)-\operatorname{div}(\boldsymbol{v})\right) d V & =\sum_{\sigma=\left(\mathrm{D} \mid \mathrm{D}^{\prime}\right)}\left(q_{\mathrm{D}}-q_{\mathrm{D}^{\prime}}\right)|\sigma| \mathbf{n}_{\mathrm{D}, \sigma} \cdot \boldsymbol{R}_{\sigma}(\boldsymbol{v}) \\
& \leq|q|_{h}\left(\sum_{\sigma=\left(\mathrm{D} \mid \mathrm{D}^{\prime}\right)} \frac{2|\sigma|^{2}}{h_{\mathrm{D}}+h_{\mathrm{D}^{\prime}}}\left|\boldsymbol{R}_{\sigma}(\boldsymbol{v})\right|^{2}\right)^{\frac{1}{2}} .
\end{aligned}
$$

Substituting (90) and (89) into (91) yields:

$$
\boldsymbol{R}_{\sigma}(\boldsymbol{v})=\boldsymbol{v}_{\sigma}^{I}-\boldsymbol{v}_{\sigma}=\frac{1}{3} \sum_{j=1}^{3}\left(\boldsymbol{v}_{i_{j}}^{I}-\boldsymbol{v}_{\sigma}\right),
$$

and after applying Jensen inequality we have that

$$
\left|\boldsymbol{R}_{\sigma}(\boldsymbol{v})\right|^{2} \leq \frac{1}{3} \sum_{j=1}^{3}\left|\boldsymbol{v}_{i_{j}}^{I}-\boldsymbol{v}_{\sigma}\right|^{2} .
$$

Finally, we apply Lemma A.1, cf. final appendix, to every difference $\left|\boldsymbol{v}_{i_{j}}^{I}-\boldsymbol{v}_{\sigma}\right|$. Note, indeed, that index $i_{j}$ for $j=1,2,3$ corresponds to a control volume of one of the meshes in $\mathfrak{M}_{h}^{\mathcal{T}}$, and that $\sigma=\mathcal{T}_{i_{1} i_{2} i_{3}}$ is inside this control volume. For instance, if $i_{j}=\mathrm{K}$, we consider the cell $\mathrm{p}_{\mathrm{K}}$ and we have that:

$$
\begin{aligned}
\left|\boldsymbol{v}_{\mathrm{K}}^{I}-\boldsymbol{v}_{\sigma}\right|^{2} & =\left|\frac{1}{m_{\mathrm{K}}|\sigma|} \int_{\boldsymbol{x} \in \mathrm{p}_{\mathrm{K}}} \int_{\boldsymbol{z} \in \sigma}(\boldsymbol{v}(\boldsymbol{x})-\boldsymbol{v}(\boldsymbol{z})) d V(\boldsymbol{x}) d S(\boldsymbol{z})\right|^{2} \\
& \leq C_{18} \frac{\operatorname{diam}\left(\mathrm{p}_{\mathrm{K}}\right)}{|\sigma|} \int_{\mathfrak{p}_{\mathrm{K}}}\left|\nabla_{x} \boldsymbol{v}(\boldsymbol{x})\right|^{2} d V(\boldsymbol{x}) .
\end{aligned}
$$

We substitute (95) into (94) and the resulting inequality into (93). Then, we use the scalings with respect to $h$ reported in consequence $\left(\mathrm{M}_{2}\right)$, i.e., inequality (17) for $\operatorname{diam}\left(\mathrm{p}_{\mathrm{K}}\right),(15)$ for $|\sigma|$, and (16) for $h_{\mathrm{D}}+h_{\mathrm{D}^{\prime}}$, to obtain

$$
\left(\sum_{\sigma=\left(\mathrm{D} \mid \mathrm{D}^{\prime}\right)} \frac{2|\sigma|^{2}}{h_{\mathrm{D}}+h_{\mathrm{D}^{\prime}}}\left|\boldsymbol{R}_{\sigma}(\boldsymbol{v})\right|^{2}\right)^{\frac{1}{2}} \leq C_{8} h\|\boldsymbol{v}\|_{H^{1}(\Omega)},
$$


where $C_{8}=\left(8 \widetilde{\mathcal{N}} C_{18} / 3\right)^{1 / 2}$, and $\widetilde{\mathcal{N}}$ is the same integer constant introduced at the end of the proof of Lemma 10. The lemma statement follows by using inequality (96) in (93).

\section{Convergence Analysis}

The main results of this paper are in this section where we prove the uniform stability and wellposedness of the scheme, cf. Theorem 1 and Corollary 1, where we derive a priori estimates for the approximation errors in the discrete and continuous setting, cf. Theorems 2 and 3.

\subsection{Stability and well-posedness}

In this subsection we prove the uniform stability of the numerical method by proving the inf-sup condition [14] that is used in the convergence analysis of the next sub-section. Let us first introduce the bilinear form for the ordered pairs of the linear space $\mathfrak{T}_{h}^{3} \times \mathfrak{D}_{h}$ :

$$
\begin{aligned}
\forall(\boldsymbol{v}, q),(\widetilde{\boldsymbol{v}}, \widetilde{q}) \in \mathfrak{T}_{h}^{3} \times \mathfrak{D}_{h}: B((\boldsymbol{v}, q) ;(\widetilde{\boldsymbol{v}}, \widetilde{q}))=[ & \left.\operatorname{div}_{h}\left(-\eta_{h} \mathrm{D}_{h}(\boldsymbol{v})+q \mathbb{I}\right), \widetilde{\boldsymbol{v}}\right]_{\mathfrak{T}_{h}} \\
& +\left[\operatorname{div}_{h}^{\mathcal{D}}(\boldsymbol{v})-\lambda h^{2} \Delta_{h}(q), \widetilde{q}\right]_{\mathfrak{D}_{h}},
\end{aligned}
$$

where $\eta_{h} \in \mathfrak{D}_{h}$ satisfies (29) and (30) and the stabilization parameter $\lambda$ is a positive real number. Note that scheme (39)-(41) can be reformulated as:

$$
\begin{aligned}
& \text { find }\left(\boldsymbol{u}_{h}, p_{h}\right) \in \mathfrak{T}_{h, 0}^{3} \times \mathfrak{D}_{h} \text { with } \sum_{D \in \mathfrak{M}_{h}^{D}} m_{D} p_{D}=0 \text { such that } \\
& \qquad B\left(\left(\boldsymbol{u}_{h}, p_{h}\right) ;(\boldsymbol{v}, q)\right)=\left[\boldsymbol{f}^{I}, \boldsymbol{v}\right]_{\mathfrak{T}_{h}} \quad \forall(\boldsymbol{v}, q) \in \mathfrak{T}_{h, 0}^{3} \times \mathfrak{D}_{h} .
\end{aligned}
$$

We can use $\boldsymbol{f}^{I}$ instead of $\boldsymbol{f}^{I, \text { int }}$ in (98) because $\boldsymbol{v}$ belongs to $\mathfrak{T}_{h, 0}^{3}$.

Theorem 1 (Inf-sup condition) For every pair $\left(\boldsymbol{u}_{h}, p_{h}\right) \in \mathfrak{T}_{h, 0}^{3} \times \mathfrak{D}_{h}$ with $p_{h}$ satisfying condition (41), i.e., $\sum_{D \in \mathfrak{M}_{h}^{D}} m_{D} p_{D}=0$, there exists a pair $(\widehat{\boldsymbol{u}}, \widehat{p}) \in \mathfrak{T}_{h, 0}^{3} \times \mathfrak{D}_{h}$ with

$$
\left\|\left|\nabla_{h} \widehat{\boldsymbol{u}}\left\|\left.\right|_{\mathfrak{D}_{h}}+\right\| \widehat{p} \|_{\mathfrak{D}_{h}}=1,\right.\right.
$$

such that there holds the uniform stability condition

$$
\left\|\left|\nabla_{h} \boldsymbol{u}_{h}\right|\right\|_{\mathfrak{D}_{h}}+\left\|\mid p_{h}\right\|_{\mathfrak{D}_{h}} \leq C_{9} B\left(\left(\boldsymbol{u}_{h}, p_{h}\right) ;(\widehat{\boldsymbol{u}}, \widehat{p})\right),
$$

where the real positive constant $C_{9}$ is independent of $h$.

Proof. Let $p_{h} \in \mathfrak{D}_{h}$ be a discrete scalar field satisfying (41). We identify $p_{h}$ and the $\mathfrak{M}_{h}^{\mathcal{D}}$-piecewise constant scalar function from $L^{2}(\Omega)$ to $\mathfrak{R}$ such that $p_{h}(\boldsymbol{x})=p_{\mathrm{D}}$ for every $\boldsymbol{x} \in \mathrm{D}$ and $\mathrm{D} \in \mathfrak{M}_{h}^{\mathcal{D}}$. Condition (41) implies that the integral of the scalar field $p_{h}$ on $\Omega$ is zero. Thus, there exist a vector field $\boldsymbol{v} \in\left(H_{0}^{1}(\Omega)\right)^{3}$ and a constant number $C_{10}>0$ such that [30]:

$$
\operatorname{div}(\boldsymbol{v})=-p_{h} \text { in } \Omega \text { and }\|\boldsymbol{v}\|_{H^{1}(\Omega)} \leq C_{10}\left\|p_{h}\right\|_{L^{2}(\Omega)} .
$$

Using the approximation property of the mean-valued interpolation operator stated in Lemma 10, cf. inequality (80), and noting that $\left\|p_{h}\right\|_{L^{2}(\Omega)}=\|\| p_{h}\|\|_{\mathfrak{D}_{h}}$ yield the useful inequality:

$$
\left\|\left.\left|\nabla_{h} \boldsymbol{v}^{I}\right|\right|_{\mathfrak{D}_{h}} \leq C_{5} C_{10}||\left|p_{h}\right|\right\|_{\mathfrak{D}_{h}} .
$$


Let us take $\widetilde{\boldsymbol{u}}=\boldsymbol{u}_{h}+\xi \boldsymbol{v}^{I}$ for some real positive number $\xi$ whose value will be specified below and $\widetilde{p}=p_{h}$. In view of (102), we have that

$$
\begin{aligned}
& \left\|\left|\nabla_{h} \widetilde{\boldsymbol{u}}\right|\right\|_{\mathfrak{D}_{h}}+\left\||| \widetilde{p}||_{\mathfrak{D}_{h}} \leq||\left|\nabla_{h} \boldsymbol{u}_{h}\left\|\left.\right|_{\mathfrak{D}_{h}}+\xi||\left|\nabla_{h} \boldsymbol{v}^{I}\right|\right\|_{\mathfrak{D}_{h}}+\left\|\left|p_{h}\right|\right\|_{\mathfrak{D}_{h}}\right.\right. \\
& \leq C_{11}\left(||\left|\nabla_{h} \boldsymbol{u}_{h}\right|||_{\mathfrak{D}_{h}}+\left|\left\|p_{h} \mid\right\|_{\mathfrak{D}_{h}}\right),\right.
\end{aligned}
$$

where we introduced the positive constant factor $C_{11}=1+C_{5} C_{10} \xi$. We will prove that, for an appropriate choice of $\xi$, condition (99) and stability inequality (100) are satisfied up to a suitable rescaling of fields $\widetilde{\boldsymbol{u}}$ and $\widetilde{p}$. Since $(\widetilde{\boldsymbol{u}}, \widetilde{p})=\left(\boldsymbol{u}_{h}, p_{h}\right)+\xi\left(\boldsymbol{v}^{I}, 0\right)$ we split the right-hand side of (100) into the sum of two terms, $\mathrm{T}_{1}$ and $\xi \mathrm{T}_{2}$,

$$
B\left(\left(\boldsymbol{u}_{h}, p_{h}\right) ;(\widetilde{\boldsymbol{u}}, \widetilde{p})\right)=B\left(\left(\boldsymbol{u}_{h}, p_{h}\right) ;\left(\boldsymbol{u}_{h}, p_{h}\right)\right)+\xi B\left(\left(\boldsymbol{u}_{h}, p_{h}\right) ;\left(\boldsymbol{v}^{I}, 0\right)\right)=\mathbf{T}_{1}+\xi \mathbf{T}_{2},
$$

which will be estimated separately. We reformulate $T_{1}$ by first using the second discrete duality relation, cf. (58), and then applying the result of Lemma 3, cf. (61) with $q=p_{h}$, to obtain:

$$
\begin{aligned}
\mathbf{T}_{1} & =\left[\operatorname{div}_{h}\left(-\eta_{h} \mathrm{D}_{h}\left(\boldsymbol{u}_{h}\right)+p_{h} \mathbb{I}\right), \boldsymbol{u}_{h}\right]_{\mathfrak{T}_{h}}+\left[\operatorname{div}_{h}^{\mathcal{D}}\left(\boldsymbol{u}_{h}\right)-\lambda h^{2} \Delta_{h}\left(p_{h}\right), p_{h}\right]_{\mathfrak{D}_{h}} \\
& =\left[\eta_{h} \mathrm{D}_{h}\left(\boldsymbol{u}_{h}\right)-p_{h} \mathbb{I}, \nabla_{h} \boldsymbol{u}_{h}\right]_{\mathfrak{D}_{h}}+\left[\operatorname{div}_{h}^{\mathcal{D}}\left(\boldsymbol{u}_{h}\right), p_{h}\right]_{\mathfrak{D}_{h}}+\lambda h^{2}\left|p_{h}\right|_{h}^{2} .
\end{aligned}
$$

Lemma 1 and compact definition (26) imply that

$$
-\left[p_{h} \mathbb{I}, \nabla_{h} \boldsymbol{u}_{h}\right]_{\mathfrak{D}_{h}}+\left[\operatorname{div}_{h}^{\mathcal{D}}\left(\boldsymbol{u}_{h}\right), p_{h}\right]_{\mathfrak{D}_{h}}=-\left[p_{h}, \operatorname{Tr}\left(\nabla_{h} \boldsymbol{u}_{h}\right)\right]_{\mathfrak{D}_{h}}+\left[\operatorname{Tr}\left(\nabla_{h} \boldsymbol{u}_{h}\right), p_{h}\right]_{\mathfrak{D}_{h}}=0,
$$

and the symmetry of the discrete operator $\mathrm{D}_{h}(\cdot)$ that

$$
\left[\eta_{h} \mathrm{D}_{h}\left(\boldsymbol{u}_{h}\right), \nabla_{h} \boldsymbol{u}_{h}\right]_{\mathfrak{D}_{h}}=\left[\eta_{h} \mathrm{D}_{h}\left(\boldsymbol{u}_{h}\right), \mathrm{D}_{h} \boldsymbol{u}_{h}\right]_{\mathfrak{D}_{h}} .
$$

Using (106) and (107) into (105), and, then, applying the left inequality of (56) provide us the lower bound for $\mathrm{T}_{1}$ :

$$
\mathbf{T}_{1}=\left[\eta_{h} \mathrm{D}_{h}\left(\boldsymbol{u}_{h}\right), \mathrm{D}_{h}\left(\boldsymbol{u}_{h}\right)\right]_{\mathfrak{D}_{h}}+\lambda h^{2}\left|p_{h}\right|_{h}^{2} \geq \underline{C}_{\eta}||\left|\mathrm{D}_{h}\left(\boldsymbol{u}_{h}\right)\right| \|_{\mathfrak{D}_{h}}^{2}+\lambda h^{2}\left|p_{h}\right|_{h}^{2} .
$$

Using the second discrete duality relation (58) allows us to split $\mathrm{T}_{2}$ as the sum of two subterms, $\mathrm{T}_{21}$ and $\mathrm{T}_{22}$, as follows:

$$
\begin{aligned}
\mathbf{T}_{2} & =\left[\operatorname{div}_{h}\left(-\eta_{h} \mathrm{D}_{h}\left(\boldsymbol{u}_{h}\right)+p_{h} \mathbb{I}\right), \boldsymbol{v}^{I}\right]_{\mathfrak{T}_{h}} \\
& =\left[\eta_{h} \mathrm{D}_{h}\left(\boldsymbol{u}_{h}\right), \nabla_{h} \boldsymbol{v}^{I}\right]_{\mathfrak{D}_{h}}-\left[p_{h} \mathbb{I}, \nabla_{h} \boldsymbol{v}^{I}\right]_{\mathfrak{D}_{h}}=\mathbf{T}_{21}+\mathbf{T}_{22} .
\end{aligned}
$$

Starting from (56) and the Cauchy-Schwarz inequality allows us to derive the chain of inequalities reported below:

$$
\begin{aligned}
\left|\mathrm{T}_{21}\right| & \leq \bar{C}_{\eta}\left\|\left|\mathrm{D}_{h}\left(\boldsymbol{u}_{h}\right)\right|\right\|_{\mathfrak{D}_{h}}\left|\left\|\nabla_{h} \boldsymbol{v}^{I} \mid\right\|_{\mathfrak{D}_{h}}\right. & & \text { [use inequality (102)] } \\
& \leq C_{5} C_{10} \bar{C}_{\eta}\left\|\left|\mathrm{D}_{h}\left(\boldsymbol{u}_{h}\right)\left\|\left.\right|_{\mathfrak{D}_{h}}\right\| p_{h} \|_{\mathfrak{D}_{h}}\right.\right. & & \text { [use Young's inequality] } \\
& \leq \widetilde{C}\left|\left\|\mathrm{D}_{h}\left(\boldsymbol{u}_{h}\right)\right\|\right|_{\mathfrak{D}_{h}}^{2}+\frac{1}{4}\left\|\left|p_{h} \|\right|_{\mathfrak{D}_{h}}^{2}=\widetilde{\mathrm{T}}_{21},\right. & &
\end{aligned}
$$

where $\widetilde{C}=\left(C_{5} C_{10} \bar{C}_{\eta}\right)^{2}$. We develop $T_{22}$ by applying the result of Lemma 1, to obtain:

$$
\begin{aligned}
-\mathrm{T}_{22} & =\left[p_{h}, \operatorname{Tr}\left(\nabla_{h} \boldsymbol{v}^{I}\right)\right]_{\mathfrak{D}_{h}} & & \text { [use inner product definition (45)] } \\
& =\sum_{\mathrm{D} \in \mathfrak{M}_{h}^{\mathcal{D}}} m_{\mathrm{D}} p_{\mathrm{D}} \operatorname{Tr}\left(\nabla_{h}^{\mathrm{D}} \boldsymbol{v}^{I}\right) & & \text { [use definition (26)] } \\
& =\sum_{\mathrm{D} \in \mathfrak{M}_{h}^{D}} m_{\mathrm{D}} p_{\mathrm{D}} \operatorname{div}_{h}^{\mathrm{D}}\left(\boldsymbol{v}^{I}\right) . & &
\end{aligned}
$$


We reformulate the summation argument as an integral on $\mathrm{D}$, we add and subtract $\int_{\mathrm{D}} \operatorname{div}(\boldsymbol{v}) d V$ and we substitute $\operatorname{div}(\boldsymbol{v})=-p_{h}$, cf. (101):

$$
\begin{aligned}
-\mathrm{T}_{22} & =\sum_{\mathrm{D} \in \mathfrak{M}_{h}^{D}}\left(\int_{\mathrm{D}} p_{\mathrm{D}}\left(\operatorname{div}_{h}^{\mathrm{D}}\left(\boldsymbol{v}^{I}\right)-\operatorname{div}(\boldsymbol{v})\right) d V+\int_{\mathrm{D}} p_{\mathrm{D}} \operatorname{div}(\boldsymbol{v}) d V\right) \\
& =\sum_{\mathrm{D} \in \mathfrak{M}_{h}^{\mathrm{D}}} \int_{\mathrm{D}} p_{\mathrm{D}}\left(\operatorname{div}_{h}^{\mathrm{D}}\left(\boldsymbol{v}^{I}\right)-\operatorname{div}(\boldsymbol{v})\right) d V-\left\|p_{h}\right\|_{\mathfrak{D}_{h}}^{2} .
\end{aligned}
$$

Using the result of Lemma 13, cf. inequality (88), yields

$$
\begin{aligned}
-\mathrm{T}_{22} & \leq C_{8} h\left|p_{h}\right| h\|\boldsymbol{v}\|_{H^{1}(\Omega)}-\left\|\left|p_{h}\right|\right\|_{\mathfrak{D}_{h}}^{2} & & \text { [use the inequality relation of (101)] } \\
& \leq C_{8} C_{10} h\left|p_{h}\right|_{h}\left\|p_{h}\left|\left\|_{\mathfrak{D}_{h}}-\right\|\right| \mid p_{h}\right\|_{\mathfrak{D}_{h}}^{2} & & \text { [use Young's inequality] } \\
& \leq\left(C_{8} C_{10}\right)^{2} h^{2}\left|p_{h}\right|_{h}^{2}-\frac{3}{4}\left\|\mid p_{h}\right\|_{\mathfrak{D}_{h}}^{2}=\widetilde{\mathrm{T}}_{22} . & &
\end{aligned}
$$

Since $\left|T_{21}\right| \leq \widetilde{T}_{21}$ implies that $T_{21} \geq-\widetilde{T}_{21}$ and $-T_{22} \leq \widetilde{T}_{22}$ implies that $T_{22} \geq-\widetilde{T}_{22}$, from (109) we have that $\mathrm{T}_{2}=\mathrm{T}_{21}+\mathrm{T}_{22} \geq-\widetilde{\mathrm{T}}_{21}-\widetilde{\mathrm{T}}_{22}$. Now, we use estimates (110) and (111) to obtain the lower bound for $T_{2}$ :

$$
\mathrm{T}_{2} \geq-\widetilde{\mathrm{T}}_{21}-\widetilde{\mathrm{T}}_{22} \geq-\left.\widetilde{C}\left|\left\|\mathrm{D}_{h}\left(\boldsymbol{u}_{h}\right)\right\|_{\mathfrak{D}_{h}}^{2}+\frac{1}{2}\left\|p_{h}\right\|_{\mathfrak{D}_{h}}^{2}-\left(C_{8} C_{10}\right)^{2} h^{2}\right| p_{h}\right|_{h} ^{2} .
$$

Collecting together the bounds for $\mathrm{T}_{1}$ and $\mathrm{T}_{2}$ gives:

$$
\mathrm{T}_{1}+\xi \mathrm{T}_{2} \geq\left(\underline{C}_{\eta}-\widetilde{C} \xi\right)\left|\left\|\mathrm{D}_{h}\left(\boldsymbol{u}_{h}\right)\right\|_{\mathfrak{D}_{h}}^{2}+\frac{\xi}{2}\left\|\left.\left|p_{h} \|_{\mathfrak{D}_{h}}^{2}+\left(\lambda-\xi\left(C_{8} C_{10}\right)^{2}\right) h^{2}\right| p_{h}\right|_{h} ^{2} .\right.\right.
$$

Let $\alpha=\min \left(\underline{C}_{\eta}, \lambda\right) / 2$. Chosing $\xi=\min \left(\left(\underline{C}_{\eta}-\alpha\right) / \widetilde{C},(\lambda-\alpha) /\left(C_{8} C_{10}\right)^{2}\right)$, so that all the constant coefficients in front of the norms are positive, eliminating the positive term containing $\left|p_{h}\right|_{h}$, and applying the discrete Korn inequality from Lemma 8 allows us to obtain the estimate:

$$
\begin{aligned}
B\left(\left(\boldsymbol{u}_{h}, p_{h}\right) ;(\widetilde{\boldsymbol{u}}, \widetilde{p})\right)=\mathrm{T}_{1}+\xi \mathrm{T}_{2} & \geq C_{12}\left(\left\|\mathrm{D}_{h}\left(\boldsymbol{u}_{h}\right)\right\|\left\|_{\mathfrak{D}_{h}}^{2}+\right\| p_{h} \|\left.\right|_{\mathfrak{D}_{h}} ^{2}\right) \\
& \geq \frac{C_{12}}{4}\left(\left\|\nabla_{h}\left(\boldsymbol{u}_{h}\right)\left|\left\|_{\mathfrak{D}_{h}}+\right\|\right| p_{h}\right\| \|_{\mathfrak{D}_{h}}\right)^{2},
\end{aligned}
$$

where the constant $C_{12}=\min \left(\underline{C}_{\eta}-\widetilde{C} \xi, \xi / 2\right)$ is independent of $h$. Let us introduce the positive factor $\gamma=\left\|\left|\nabla_{h} \widetilde{\boldsymbol{u}}\right|\right\|_{\mathfrak{D}_{h}}+\|\mid\| \widetilde{p} \|_{\mathfrak{D}_{h}}$ and use inequality (103) to obtain:

$$
\begin{aligned}
B\left(\left(\boldsymbol{u}_{h}, p_{h}\right) ;(\widetilde{\boldsymbol{u}}, \widetilde{p})\right) & \geq \frac{C_{12}}{4 C_{11}}\left(\left.\left\|\nabla_{h} \boldsymbol{u}_{h}\right\|\right|_{\mathfrak{D}_{h}}+\left.\left\|p_{h}\right\|\right|_{\mathfrak{D}_{h}}\right)\left(\left\|\left|\nabla_{h} \widetilde{\boldsymbol{u}}\right|\right\|_{\mathfrak{D}_{h}}+\|\widetilde{p}\|_{\mathfrak{D}_{h}}\right) \\
& =\frac{C_{12}}{4 C_{11}}\left(\left\|\nabla_{h} \boldsymbol{u}_{h}\right\|\left\|_{\mathfrak{D}_{h}}+\right\| p_{h} \|\left.\right|_{\mathfrak{D}_{h}}\right) \gamma
\end{aligned}
$$

Dividing both sides of (114) by $\gamma$ and using the fact that $B(\cdot ; \cdot)$ is a linear map with respect to its argument yields

$$
B\left(\left(\boldsymbol{u}_{h}, p_{h}\right) ;(\widetilde{\boldsymbol{u}} / \gamma, \widetilde{p} / \gamma)\right) \geq \frac{C_{12}}{4 C_{11}}\left(\left\|\nabla_{h} \boldsymbol{u}_{h}\right\|_{\mathfrak{D}_{h}}+\left\|p_{h}\right\|_{\mathfrak{D}_{h}}\right),
$$

which is the second theorem's inequality for $\widehat{\boldsymbol{u}}=\widetilde{\boldsymbol{u}} / \gamma$ and $\widetilde{p}=\widetilde{p} / \gamma$ if, by comparison, we set

$$
C_{9}=\frac{4 C_{11}}{C_{12}} .
$$


Note that $C_{9}$ is independent of $h$ and the first theorem's inequality is readily satisfied since

$$
\left.\left\||| \nabla_{h} \widehat{\boldsymbol{u}}\right\|\right|_{\mathfrak{D}_{h}}+\left\|\widehat { p } \left|\left\|_{\mathfrak{D}_{h}}=||\left|\nabla_{h}(\widetilde{\boldsymbol{u}} / \gamma)\left\|\left.\right|_{\mathfrak{D}_{h}}+\right\| \widetilde{p} / \gamma\right|\right\|_{\mathfrak{D}_{h}}=\frac{1}{\gamma}\left(\left\||| \nabla_{h} \widetilde{\boldsymbol{u}}\left|\left\|_{\mathfrak{D}_{h}}+\right\|\right| \mid \widetilde{p}\right\|_{\mathfrak{D}_{h}}\right)=1 .\right.\right.
$$

Corollary 1 (Well-posedness) The DDFV method provided by equations (39)-(41) admits a unique solution $\left(\boldsymbol{u}_{h}, p_{h}\right) \in \mathfrak{T}_{h, 0}^{3} \times \mathfrak{D}_{h}$ for any mesh set $\left(\mathfrak{M}_{h}^{\mathcal{T}}, \mathfrak{M}_{h}^{\mathcal{D}}\right)$ satisfying Assumptions $\left(A_{1}\right)-\left(A_{2}\right)$, any discrete viscosity field $\eta_{h} \in \mathfrak{D}_{h}$ satisfying (30) and any stabilization parameter $\lambda>0$.

Proof. Let us consider the homogeneous discrete problem given by setting $\boldsymbol{f}$, the right-hand side of (39), to zero so that $\boldsymbol{f}^{I}=0$ in (98). From (98) and the result of Theorem 1, cf. inequality (100), it follows that $\nabla_{h} \boldsymbol{u}_{h}=0$ and $p_{h}=0$. The former identity implies that the degrees of freedom of the velocity $\boldsymbol{u}_{h}$ are constant, and it is immediate to see that the homogeneous boundary condition implies that $\boldsymbol{u}_{h}=0$.

\subsection{A priori error estimates}

In this section, we derive an a priori estimate of the approximation errors for the degrees of freedom of the velocity and pressure fields solving the DDFV scheme (39)-(41). These errors are given by comparison with $\boldsymbol{u}^{J} \in \mathfrak{T}_{h}^{3}$, the center-valued interpolation of $\boldsymbol{u}$ on $\mathfrak{M}_{h}^{\mathcal{T}}$ defined in accordance with (34)(36), and $p^{I} \in \mathfrak{D}_{h}$, the mean-valued interpolation of $p$ on $\mathfrak{M}_{h}^{\mathcal{D}}$ defined in accordance with (37). The result is stated and proved in Theorem 2. The DDFV approximation to the Stokes velocity, its gradient and the scalar pressure field in the continuous setting are defined through the identification of the discrete fields in $\mathfrak{D}_{h}$ with the piecewise constant fields taking the same values on the cells of mesh $\mathfrak{M}_{h}^{\mathcal{D}}$. In Theorem 3, we prove an a priori estimate for these approximations.

Theorem 2 Let $(\boldsymbol{u}, p) \in\left(H^{2}(\Omega)\right)^{3} \times H^{1}(\Omega)$ be the velocity and pressure solution fields of the steady Stokes problem (1)-(3) under hypothesis $\left(H_{1}\right)-\left(H_{3}\right)$, and such that $p$ satisfies the compatibility condition (6). Let $\left(\boldsymbol{u}_{h}, p_{h}\right) \in \mathfrak{T}_{h, 0}^{3} \times \mathfrak{D}_{h}$ be the DDFV approximations to velocity and pressure that solve the scheme's equations (39)-(41) under Assumptions $\left(A_{1}\right)-\left(A_{2}\right)$. Let $\boldsymbol{u}^{J} \in \mathfrak{T}_{h, 0}^{3}$ be the center-valued interpolation of $\boldsymbol{u}$ on $\mathfrak{M}_{h}^{\mathcal{T}}$ defined in accordance with (34)-(36), and $p^{I} \in \mathfrak{D}_{h}$ be the mean-valued interpolation of $p$ on $\mathfrak{M}_{h}^{\mathcal{D}}$ defined in accordance with (37).

Then, there exists a real positive constant $C_{13}$ independent of $h$ such that

$$
\left\|\left|\nabla_{h}\left(\boldsymbol{u}^{J}-\boldsymbol{u}_{h}\right)\right|\right\|_{\mathfrak{D}_{h}}+\left\|\left|p^{I}-p_{h} \|\right|_{\mathfrak{D}_{h}} \leq C_{13} h\left(\|\boldsymbol{u}\|_{H^{2}(\Omega)}+\|p\|_{H^{1}(\Omega)}\right) .\right.
$$

Proof. The proof starts from the stability condition of Theorem 1. Let $\mathbf{e}_{h}=\boldsymbol{u}^{J}-\boldsymbol{u}_{h} \in \mathfrak{T}_{h, 0}^{3}$ denote the approximation error for the velocity solution field and $\varepsilon_{h}=p^{I}-p_{h} \in \mathfrak{D}_{h}$ the approximation error for the pressure solution field. Theorem 1 implies the existence of two discrete fields $(\boldsymbol{v}, q) \in \mathfrak{T}_{h, 0}^{3} \times \mathfrak{D}_{h}$ such that

$$
\left|\left\|\nabla _ { h } \boldsymbol { v } \left|\left\|_{\mathfrak{D}_{h}}+|\|q\||_{\mathfrak{D}_{h}}=1\right.\right.\right.\right.
$$

and

$$
\left\|\left|\nabla_{h} \mathbf{e}_{h}\left\|_{\mathfrak{D}_{h}}+\right\|\right| \varepsilon_{h}\right\|_{\mathfrak{D}_{h}} \leq C_{9} B\left(\left(\mathbf{e}_{h}, \varepsilon_{h}\right) ;(\boldsymbol{v}, q)\right) .
$$

Using the definition of $B(\cdot, \cdot)$, cf. equation (97), the definition of the approximation errors $\mathbf{e}_{h}$ and $\varepsilon_{h}$, scheme's equations (39) and (40), and substituting $\boldsymbol{f}^{I}$, the mean-valued interpolation of the loading 
term $\boldsymbol{f}$, with the mean-valued interpolation of the left-hand side of equation (1), yield:

$$
\begin{aligned}
B\left(\left(\mathbf{e}_{h}, \varepsilon_{h}\right) ;(\boldsymbol{v}, q)\right)=\left[\operatorname{div}_{h}\left(-\eta_{h} \mathrm{D}_{h}\left(\mathbf{e}_{h}\right)+\varepsilon_{h} \mathbb{I}\right), \boldsymbol{v}\right]_{\mathfrak{T}_{h}}+\left[\operatorname{div}_{h}^{\mathcal{D}}\left(\mathbf{e}_{h}\right)-\lambda h^{2} \Delta_{h}\left(\varepsilon_{h}\right), q\right]_{\mathfrak{D}_{h}} \\
=\left[\operatorname{div}_{h}\left(-\eta_{h} \mathrm{D}_{h}\left(\boldsymbol{u}^{J}\right)+p^{I} \mathbb{I}\right)-\boldsymbol{f}^{I}, \boldsymbol{v}\right]_{\mathfrak{T}_{h}}+\left[\operatorname{div}_{h}^{\mathcal{D}}\left(\boldsymbol{u}^{J}\right)-\lambda h^{2} \Delta_{h}\left(p^{I}\right), q\right]_{\mathfrak{D}_{h}} \\
=\left[\operatorname{div}_{h}\left(-\eta_{h} \mathrm{D}_{h}\left(\boldsymbol{u}^{J}\right)+p^{I} \mathbb{I}\right)-(\operatorname{div}(-\eta \mathrm{D}(\boldsymbol{u})+p \mathbb{I}))^{I}, \boldsymbol{v}\right]_{\mathfrak{T}_{h}} \\
\quad+\left[\operatorname{div}_{h}^{\mathcal{D}}\left(\boldsymbol{u}^{J}\right)-\lambda h^{2} \Delta_{h}\left(p^{I}\right), q\right]_{\mathfrak{D}_{h}} .
\end{aligned}
$$

To ease notation, we introduce the symbols:

$$
\psi_{h}=-\eta_{h} \mathrm{D}_{h}\left(\boldsymbol{u}^{J}\right)+p^{I} \mathbb{I} \quad \text { and } \quad \psi=-\eta \mathrm{D}(\boldsymbol{u})+p \mathbb{I} .
$$

We also consider the discrete tensor field $\widetilde{\psi}=\left\{\left(\widetilde{\psi}_{\mathrm{D}}\right)\right\} \in \mathfrak{D}_{h}^{3 \times 3}$, which is uniquely defined on each diamond cell $\mathrm{D} \in \mathfrak{M}_{h}^{\mathcal{D}}$ by the three constant vectors $\left(\widetilde{\psi}_{\mathrm{D}} \mathbf{N}_{\mathrm{K}, \mathrm{L}}, \widetilde{\psi}_{\mathrm{D}} \mathbf{N}_{\mathrm{A}, \mathrm{B}}, \widetilde{\psi}_{\mathrm{D}} \mathbf{N}_{\mathrm{E}, \mathrm{F}}\right)$ through the formulas

$$
\begin{aligned}
\widetilde{\psi}_{\mathrm{D}} \mathbf{N}_{\mathrm{K}, \mathrm{L}}=\int_{\mathcal{S}_{\mathrm{D}, \mathrm{KL}}} \psi \mathbf{n} d S, & & {\left[\text { using } \mathcal{S}_{\mathrm{D}, \mathrm{KL}} \text { defined in }(7)\right] } \\
\widetilde{\psi}_{\mathrm{D}} \mathbf{N}_{\mathrm{A}, \mathrm{B}}=\int_{\mathcal{S}_{\mathrm{D}, \mathrm{AB}}} \psi \mathbf{n} d S, & & {\left[\text { using } \mathcal{S}_{\mathrm{D}, \mathrm{AB}} \text { defined in }(9)\right] } \\
\widetilde{\psi}_{\mathrm{D}} \mathbf{N}_{\mathrm{E}, \mathrm{F}}=\int_{\mathcal{S}_{\mathrm{D}, \mathrm{EF}}} \psi \mathbf{n} d S, & & {\left[\text { using } \mathcal{S}_{\mathrm{D}, \mathrm{EF}} \text { defined in }(11)\right] }
\end{aligned}
$$

$\mathbf{n}$ being the unit vector orthogonal to the surfaces $\mathcal{S}_{\mathrm{D}, \mathrm{KL}}, \mathcal{S}_{\mathrm{D}, \mathrm{AB}}$, and $\mathcal{S}_{\mathrm{D}, \mathrm{EF}}$, over which these integrals are defined. By construction, it follows that

$$
(\operatorname{div}(\psi))^{I}=\operatorname{div}_{h}(\widetilde{\psi}) .
$$

In fact, after recalling (13) and (31), for every $\mathrm{p} \in \mathfrak{M}_{h}^{\mathcal{P}}$ we have that

$$
\begin{aligned}
\operatorname{div}_{h}^{\mathrm{p}}(\widetilde{\psi}) & =\frac{1}{m_{\mathrm{p}}} \sum_{\mathrm{D} \in \mathfrak{M}_{h}^{\mathcal{D}}} \widetilde{\psi}_{\mathrm{D}} \mathbf{N}_{\mathrm{D}, \mathrm{p}}=\frac{1}{m_{\mathrm{p}}} \sum_{\mathrm{D} \in \mathfrak{M}_{h}^{\mathcal{D}} \mid} \int_{\mathcal{S}_{\mathrm{D}, K L}} \psi \mathbf{n} d S=\frac{1}{m_{\mathrm{p}}} \int_{\partial \mathrm{p}} \psi \mathbf{n} d S \\
& =\frac{1}{m_{\mathrm{p}}} \int_{\mathrm{p}} \operatorname{div}(\psi) d V=\mathbb{P}_{\mathrm{M}}^{\mathrm{p}}(\operatorname{div}(\psi)),
\end{aligned}
$$

and a similar $\operatorname{argument}$ holds for $\operatorname{div}_{h}^{v}(\widetilde{\psi})$ and $\operatorname{div}_{h}^{\mathbf{s}}(\widetilde{\psi})$, i.e., for the elements of the triplet $(20)$ that provides the discrete divergence of $\widetilde{\psi}$ on the mesh set $\mathfrak{M}_{h}^{\mathcal{T}}:=\left(\mathfrak{M}_{h}^{\mathcal{P}}, \mathfrak{M}_{h}^{\mathcal{V}}, \mathfrak{M}_{h}^{\mathcal{E F}}\right)$ in accordance with equations (20). Now, we substitute (122) into (120), and, then, we apply the second duality relation (58) to split the bilinear form through the sum of three terms, namely $T_{1}, T_{2}$, and $T_{3}$ :

$$
\begin{aligned}
B\left(\left(\mathbf{e}_{h}, \varepsilon_{h}\right) ;(\boldsymbol{v}, q)\right) & =\left[\operatorname{div}_{h}\left(\psi_{h}-\widetilde{\psi}\right), \boldsymbol{v}\right]_{\mathfrak{T}_{h}}+\left[\operatorname{div}_{h}^{\mathcal{D}}\left(\boldsymbol{u}^{J}\right)-\lambda h^{2} \Delta_{h}\left(p^{I}\right), q\right]_{\mathfrak{D}_{h}} \\
& =-\left[\psi_{h}-\widetilde{\psi}, \nabla_{h} \boldsymbol{v}\right]_{\mathfrak{D}_{h}}+\left[\operatorname{div}_{h}^{\mathcal{D}}\left(\boldsymbol{u}^{J}\right), q\right]_{\mathfrak{D}_{h}}-\left[\lambda h^{2} \Delta_{h}\left(p^{I}\right), q\right]_{\mathfrak{D}_{h}} \\
& =\mathbf{T}_{1}+\mathbf{T}_{2}+\mathbf{T}_{3} .
\end{aligned}
$$

The theorem's statement, i.e., inequality (117), follows from deriving an appropriate upper bound for these three terms.

Estimate of term $T_{1}$

Using Cauchy-Schwarz inequality and noting that relation (118) implies that $\left\|\left|\nabla_{h} \boldsymbol{v}\right|\right\|_{\mathfrak{D}_{h}} \leq 1$ yield:

$$
\left|\mathrm{T}_{1}\right| \leq\left\||| \psi_{h}-\widetilde{\psi}\left|\left\|_{\mathfrak{D}_{h}}\right\|\right| \nabla_{h} \boldsymbol{v}\left|\left\|_{\mathfrak{D}_{h}} \leq\right\|\right|\left|\psi_{h}-\widetilde{\psi}\right|\right\|_{\mathfrak{D}_{h}} .
$$


In order to estimate the right-hand side of (125), we define $\mathbf{n}_{\mathrm{K}, \mathrm{L}}=\mathbf{N}_{\mathrm{K}, \mathrm{L}} /\left|\mathbf{N}_{\mathrm{K}, \mathrm{L}}\right|, \mathbf{n}_{\mathrm{A}, \mathrm{B}}=\mathbf{N}_{\mathrm{A}, \mathrm{B}} /$ $\left|\mathbf{N}_{\mathrm{A}, \mathrm{B}}\right|$ and $\mathbf{n}_{\mathrm{E}, \mathrm{F}}=\mathbf{N}_{\mathrm{E}, \mathrm{F}} /\left|\mathbf{N}_{\mathrm{E}, \mathrm{F}}\right|$. Using definition (51) gives:

$$
||\left|\psi_{h}-\widetilde{\psi}\right| \|_{\mathfrak{D}_{h}}^{2} \leq C_{14} \sum_{\mathrm{D} \in \mathfrak{M}_{h}^{D}} m_{\mathrm{D}}\left(\left|\left(\psi_{h}-\widetilde{\psi}\right) \mathbf{n}_{\mathrm{K}, \mathrm{L}}\right|^{2}+\left|\left(\psi_{h}-\widetilde{\psi}\right) \mathbf{n}_{\mathrm{A}, \mathrm{B}}\right|^{2}+\left|\left(\psi_{h}-\widetilde{\psi}\right) \mathbf{n}_{\mathrm{E}, \mathrm{F}}\right|^{2}\right),
$$

where $C_{14}$ does not depend on $h$.

Since $\psi_{h \mid \mathrm{D}}$ is constant on each diamond cell $\mathrm{D}$, we rewrite the argument of the summation of the right-hand side of (126) as a summation over the planar subfaces forming $\mathcal{S}_{\mathrm{D}}=\mathcal{S}_{\mathrm{D}, \mathrm{KL}} \cup \mathcal{S}_{\mathrm{D}, \mathrm{AB}} \cup \mathcal{S}_{\mathrm{D}, \mathrm{EF}}$. We denote the summation index by $\sigma \subset \mathcal{S}_{\mathrm{D}}$. Then, by using Jensen inequality and the definition of $\psi_{h}$ and $\psi$ we obtain:

$$
\begin{aligned}
\mid\left(\psi_{h}-\widetilde{\psi}\right) & \left.\mathbf{n}_{\mathrm{K}, \mathrm{L}}\right|^{2}+\left|\left(\psi_{h}-\widetilde{\psi}\right) \mathbf{n}_{\mathrm{A}, \mathrm{B}}\right|^{2}+\left|\left(\psi_{h}-\widetilde{\psi}\right) \mathbf{n}_{\mathrm{E}, \mathrm{F}}\right|^{2} \\
& \leq \sum_{\sigma \subset \mathcal{S}_{\mathrm{D}}}\left|\frac{1}{\sigma} \int_{\sigma}\left(\psi_{h}-\psi\right) \mathbf{n} d S\right|^{2} \leq \sum_{\sigma \subset \mathcal{S}_{\mathrm{D}}} \frac{1}{|\sigma|} \int_{\sigma}\left|\psi_{h}-\psi\right|^{2} d S \\
& \leq 2 \sum_{\sigma \subset \mathcal{S}_{\mathrm{D}}} \frac{1}{|\sigma|}\left(\left\|\eta_{h} \mathrm{D}_{h}\left(\boldsymbol{u}^{J}\right)-\eta \mathrm{D}(\boldsymbol{u})\right\|_{L^{2}(\sigma)}^{2}+\left\|p^{I}-p\right\|_{L^{2}(\sigma)}^{2}\right) \\
& =\mathrm{T}_{11}^{\mathrm{D}}+\mathrm{T}_{12}^{\mathrm{D}} .
\end{aligned}
$$

To get the upper bound for $\mathrm{T}_{11}^{\mathrm{D}}$, we add and subtract $\eta \mathrm{D}_{h}\left(\boldsymbol{u}^{J}\right)$ and use the Cauchy-Schwarz inequality:

$$
\left\|\eta_{h} \mathrm{D}_{h}\left(\boldsymbol{u}^{J}\right)-\eta \mathrm{D}(\boldsymbol{u})\right\|_{L^{2}(\sigma)}^{2} \leq 2\left\|\left(\eta_{h}-\eta\right) \mathrm{D}_{h}\left(\boldsymbol{u}^{J}\right)\right\|_{L^{2}(\sigma)}^{2}+2\left\|\eta\left(\mathrm{D}_{h}\left(\boldsymbol{u}^{J}\right)-\mathrm{D}(\boldsymbol{u})\right)\right\|_{L^{2}(\sigma)}^{2} .
$$

The first term in the right-hand side of (128) is bounded by using the approximation property of $\eta_{h}$ given by (29), Agmon inequality and noting that the $H^{1}$-seminorm of $\mathrm{D}_{h}\left(\boldsymbol{u}^{J}\right)$ is zero because $\mathrm{D}_{h}\left(\boldsymbol{u}^{J}\right)$ is constant on D, and applying the left-most inequality in (72) and inequality (81). Thus, we have that

$$
\begin{aligned}
\left\|\left(\eta_{h}-\eta\right) \mathrm{D}_{h}\left(\boldsymbol{u}^{J}\right)\right\|_{L^{2}(\sigma)}^{2} & \leq\left\|\eta_{h}-\eta\right\|_{L^{\infty}(\mathrm{D})}^{2}\left\|\mathrm{D}_{h}\left(\boldsymbol{u}^{J}\right)\right\|_{L^{2}(\sigma)}^{2} \\
& \leq \widetilde{C}_{\eta}^{2} C_{A g} h_{\mathrm{D}}^{2}\left(h_{\mathrm{D}}^{-1}\left\|\mathrm{D}_{h}\left(\boldsymbol{u}^{J}\right)\right\|_{L^{2}(\mathrm{D})}^{2}+h_{\mathrm{D}}\left|\mathrm{D}_{h}\left(\boldsymbol{u}^{J}\right)\right|_{H^{1}(\mathrm{D})}^{2}\right) \\
& \leq \widetilde{C}_{\eta}^{2} C_{A g} h_{\mathrm{D}}\left\|\nabla_{h} \boldsymbol{u}^{J}\right\|_{L^{2}(\mathrm{D})}^{2} \leq \widetilde{C}_{\eta}^{2} C_{A g} C_{5}^{2} h_{\mathrm{D}}\|\boldsymbol{u}\|_{H^{2}(\mathrm{D})}^{2} .
\end{aligned}
$$

To get a bound for the second term in the right-hand side of (128), let us consider $\mathrm{D}(\boldsymbol{u})_{\mid \mathrm{D}}^{I}$, the average of the derivative $\mathrm{D}(\boldsymbol{u})$ on the diamond cell $\mathrm{D}$, which is defined in accordance with (38). Then, by using (5), adding and subtracting $\mathrm{D}(\boldsymbol{u})_{\mid \mathrm{D}}^{I}$, and applying Cauchy-Schwarz inequality we obtain:

$$
\begin{aligned}
\left\|\eta\left(\mathrm{D}_{h}\left(\boldsymbol{u}^{J}\right)-\mathrm{D}(\boldsymbol{u})\right)\right\|_{L^{2}(\sigma)}^{2} \leq & 2 \bar{C}_{\eta}^{2}\left(\left\|\mathrm{D}_{h}\left(\boldsymbol{u}^{J}\right)-\mathrm{D}(\boldsymbol{u})_{\mid \mathrm{D}}^{I}\right\|_{L^{2}(\sigma)}^{2}\right. \\
& \left.+\left\|\mathrm{D}(\boldsymbol{u})_{\mid \mathrm{D}}^{I}-\mathrm{D}(\boldsymbol{u})\right\|_{L^{2}(\sigma)}^{2}\right) .
\end{aligned}
$$

The first term in the right-hand side of (130) is controlled through Agmon inequality, noting that the $H^{1}$-seminorm of $\left(\mathrm{D}_{h}\left(\boldsymbol{u}^{J}\right)-\mathrm{D}(\boldsymbol{u})_{\mid \mathrm{D}}^{I}\right)$ is zero since this term is constant on $\mathrm{D}$, and applying the estimate of the interpolation error provided by Lemma 12, cf. inequality (87). We obtain the following 
development:

$$
\begin{aligned}
\left\|\mathrm{D}_{h}\left(\boldsymbol{u}^{J}\right)-\mathrm{D}(\boldsymbol{u})_{\mid \mathrm{D}}^{I}\right\|_{L^{2}(\sigma)}^{2} \leq & C_{A g}\left(h_{\mathrm{D}}^{-1}\left\|\mathrm{D}_{h}\left(\boldsymbol{u}^{J}\right)-\mathrm{D}(\boldsymbol{u})_{\mid \mathrm{D}}^{I}\right\|_{L^{2}(\mathrm{D})}^{2}\right. \\
& \left.+h_{\mathrm{D}}\left|\mathrm{D}_{h}\left(\boldsymbol{u}^{J}\right)-\mathrm{D}(\boldsymbol{u})_{\mid \mathrm{D}}^{I}\right|_{H^{1}(\mathrm{D})}^{2}\right) \\
\leq & C_{A g} h_{\mathrm{D}}^{-1}\left\|\mathrm{D}_{h}\left(\boldsymbol{u}^{J}\right)-\mathrm{D}(\boldsymbol{u})_{\mid \mathrm{D}}^{I}\right\|_{L^{2}(\mathrm{D})}^{2} \\
\leq & C_{A g} C_{7}^{2} h_{\mathrm{D}}\|\boldsymbol{u}\|_{H^{2}(\mathrm{D})}^{2} .
\end{aligned}
$$

The second term in the right-hand side of (130) is controlled through Agmon inequality and applying the estimate of the interpolation error provided by Lemma 7 , cf. inequality (71):

$$
\begin{aligned}
\left\|\mathrm{D}(\boldsymbol{u})_{\mid \mathrm{D}}^{I}-\mathrm{D}(\boldsymbol{u})\right\|_{L^{2}(\sigma)}^{2} & \leq C_{A g}\left(h_{\mathrm{D}}^{-1}\left\|\mathrm{D}(\boldsymbol{u})_{\mid \mathrm{D}}^{I}-\mathrm{D}(\boldsymbol{u})\right\|_{L^{2}(\mathrm{D})}^{2}+h_{\mathrm{D}}\|\mathrm{D}(\boldsymbol{u})\|_{H^{1}(\mathrm{D})}^{2}\right) \\
& \leq C_{A g}\left(1+C_{3}^{2}\right) h_{\mathrm{D}}\|\boldsymbol{u}\|_{H^{2}(\mathrm{D})}^{2} .
\end{aligned}
$$

Substituting (132) and (131) into (130), and, then, the resulting inequality and (129) into (128) give us the bound for $T_{11}^{\mathrm{D}}$ :

$$
\mathrm{T}_{11}^{\mathrm{D}} \leq C_{15} h_{\mathrm{D}}^{-1}\|\boldsymbol{u}\|_{H^{2}(\mathrm{D})}^{2}
$$

where the real positive constant $C_{15}$ absorbs all the previous constants and the scaling coefficients that depend on $C_{r e g}$ according to consequence $\left(\mathrm{M}_{2}\right)$.

Similarly, to get a bound for $\mathrm{T}_{12}^{\mathrm{D}}$ we apply Agmon inequality, note that the $H^{1}$-seminorm of $p^{I}$ is zero because $p^{I}{ }_{\mid \mathrm{D}}$ is constant on $\mathrm{D}$, and use the estimate of the interpolation error given by Lemma 7 , cf. inequality (71). Therefore, for every $\sigma \subset \mathcal{S}_{\mathrm{D}}$ and each $\mathrm{D} \in \mathfrak{M}_{h}^{\mathcal{D}}$, there holds that:

$$
\left\|p^{I}-p\right\|_{L^{2}(\sigma)}^{2} \leq C_{A g}\left(h_{\mathrm{D}}^{-1}\left\|p^{I}-p\right\|_{L^{2}(\mathrm{D})}^{2}+h_{\mathrm{D}}|p|_{H^{1}(\mathrm{D})}^{2}\right) \leq C_{A g}\left(1+C_{3}^{2}\right) h_{\mathrm{D}}\|p\|_{H^{1}(\mathrm{D})}^{2} .
$$

Using (134) and noting that condition (15) from $\left(\mathrm{M}_{2}\right)$ implies that $C_{\text {reg }}^{2} h_{\mathrm{D}}^{2} \leq|\sigma|$ allows us to derive the following bound for $\mathrm{T}_{12}^{\mathrm{D}}$ :

$$
\begin{aligned}
\mathrm{T}_{12}^{\mathrm{D}} & =2 \sum_{\sigma \subset \mathcal{S}_{\mathrm{D}}} \frac{1}{|\sigma|}\left\|p^{I}-p\right\|_{L^{2}(\sigma)}^{2} \leq 2 C_{A g}\left(1+C_{3}^{2}\right) \sum_{\sigma \subset \mathcal{S}_{\mathrm{D}}} \frac{1}{|\sigma|} h_{\mathrm{D}}\|p\|_{H^{1}(\mathrm{D})}^{2} \\
& \leq 6\left(C_{A g}\left(1+C_{3}^{2}\right) / C_{r e g}^{2}\right) h_{\mathrm{D}}^{-1}\|p\|_{H^{1}(\mathrm{D})}^{2} .
\end{aligned}
$$

Using the obvious fact that $m_{\mathrm{D}} \leq h_{\mathrm{D}}^{3} \leq h^{3}$ and introducing a "cumulative" constant $C_{16}$ to take into consideration all constant factors, we obtain:

$$
\begin{aligned}
\left\|\psi_{h}-\widetilde{\psi}\right\|_{\mathfrak{D}_{h}}^{2} & \leq C_{16} \sum_{\mathrm{D} \in \mathfrak{M}_{h}^{\mathcal{D}}} m_{\mathrm{D}} h_{\mathrm{D}}^{-1}\left(\|\boldsymbol{u}\|_{H^{2}(\mathrm{D})}^{2}+\|p\|_{H^{1}(\mathrm{D})}^{2}\right) \\
& \leq C_{16} h^{2} \sum_{\mathrm{D} \in \mathfrak{M}_{h}^{D}}\left(\|\boldsymbol{u}\|_{H^{2}(\mathrm{D})}^{2}+\|p\|_{H^{1}(\mathrm{D})}^{2}\right) \\
& =C_{16} h^{2}\left(\|\boldsymbol{u}\|_{H^{2}(\Omega)}^{2}+\|p\|_{H^{1}(\Omega)}^{2}\right),
\end{aligned}
$$

which implies that

$$
\left|\mathrm{T}_{1}\right| \leq C_{16} h\left(\|\boldsymbol{u}\|_{H^{2}(\Omega)}+\|p\|_{H^{1}(\Omega)}\right)
$$




\section{Estimate of term $\mathrm{T}_{2}$}

The estimate of term $\mathrm{T}_{2}$ follows from the application of Cauchy-Schwarz inequality and Lemma 11, cf. inequality (86),

$$
\left|\mathrm{T}_{2}\right|=\|\| \operatorname{div}_{h}^{\mathcal{D}}\left(\boldsymbol{u}^{J}\right)\left\|_{\mathfrak{D}_{h}}\right\| q\|\|_{\mathfrak{D}_{h}} \leq C_{6} h\|\boldsymbol{u}\|_{H^{2}(\Omega)},
$$

because relation (118) implies that \|\|$q \|\left.\right|_{\mathfrak{D}_{h}} \leq 1$.

Estimate of term $\mathrm{T}_{3}$

We reorder the summation in term $\mathrm{T}_{3}$ and use the explicit form of the discrete Laplacian operator (28) applied to the interpolation field $p^{I}$ to obtain:

$$
\begin{aligned}
& \mathrm{T}_{3}=-\lambda h^{2} \sum_{\mathrm{D} \in \mathfrak{M}_{h}^{\mathcal{D}}} q_{\mathrm{D}} \sum_{\mathrm{D}^{\prime} \in \mathfrak{M}_{h}^{\mathcal{D}} \mid \mathrm{D}} \frac{h_{\mathrm{D}}+h_{\mathrm{D}^{\prime}}}{2}\left(p_{\mathrm{D}^{\prime}}^{I}-p_{\mathrm{D}}^{I}\right) \quad \text { [reorder summation] } \\
& \left.=\lambda h^{2} \sum_{\left(\mathrm{D} \mid \mathrm{D}^{\prime}\right)} \frac{h_{\mathrm{D}}+h_{\mathrm{D}^{\prime}}}{2}\left(p_{\mathrm{D}^{\prime}}^{I}-p_{\mathrm{D}}^{I}\right)\left(q_{\mathrm{D}^{\prime}}-q_{\mathrm{D}}\right) \quad \text { [use Cauchy-Schwarz inequality }\right] \\
& \leq \lambda h^{2}\left(\sum_{\left(\mathrm{D} \mid \mathrm{D}^{\prime}\right)} \frac{h_{\mathrm{D}}+h_{\mathrm{D}^{\prime}}}{2}\left|p_{\mathrm{D}^{\prime}}^{I}-p_{\mathrm{D}}^{I}\right|^{2}\right)^{1 / 2}\left(\sum_{\left(\mathrm{D} \mid \mathrm{D}^{\prime}\right)} \frac{h_{\mathrm{D}}+h_{\mathrm{D}^{\prime}}}{2}\left|q_{\mathrm{D}^{\prime}}-q_{\mathrm{D}}\right|^{2}\right)^{1 / 2} \quad[\text { use }(60)] \\
& =\lambda h^{2}\left|p^{I}\right|_{h}|q|_{h} .
\end{aligned}
$$

Using Young's inequality, the scaling properties of consequence $\left(\mathrm{M}_{2}\right)$ and noting again that $\|q\| \|_{\mathfrak{D}_{h}} \leq 1$ due to relation (118) yield:

$$
\begin{aligned}
|q|_{h}^{2} & =\sum_{\left(\mathrm{D} \mid \mathrm{D}^{\prime}\right)} \frac{h_{\mathrm{D}}+h_{\mathrm{D}^{\prime}}}{2}\left|q_{\mathrm{D}^{\prime}}-q_{\mathrm{D}}\right|^{2} \leq 2 \sum_{\left(\mathrm{D} \mid \mathrm{D}^{\prime}\right)} \frac{h_{\mathrm{D}}+h_{\mathrm{D}^{\prime}}}{2}\left(\left|q_{\mathrm{D}^{\prime}}\right|^{2}+\left|q_{\mathrm{D}}\right|^{2}\right) \\
& \leq \frac{8}{C_{\text {reg }}^{3}} h^{-2} \sum_{\mathrm{D} \in \mathfrak{M}_{h}^{\mathcal{D}}} m_{\mathrm{D}}\left|q_{\mathrm{D}}\right|^{2}=\frac{8}{C_{\text {reg }}^{3}} h^{-2} \mid\|q\|_{\mathfrak{D}_{h}}^{2} \leq \frac{8}{C_{r e g}^{3}} h^{-2} .
\end{aligned}
$$

Taking the square root of inequality (140), substituting the result into (139) and using the result of Lemma 9, cf. inequality (75), provide us with the following bound:

$$
\left|\mathrm{T}_{3}\right| \leq \frac{2 \sqrt{2} C_{4}}{C_{\text {reg }}^{3 / 2}} \lambda h|p|_{H^{1}(\Omega)} .
$$

Error estimate (117) eventually follows by considering estimates (137), (138), and (141) into (124), using the result in stability inequality(119), and properly defining the constant factor $C_{13}$, which is independent of $h$.

As previously discussed, we identify the discrete tensor field $\nabla_{h} \boldsymbol{u}_{h} \in \mathfrak{D}_{h}^{3 \times 3}$ with the piecewise constant tensor field $\nabla_{h} \boldsymbol{u}_{h}(\boldsymbol{x})=\nabla_{h}^{\mathrm{D}} \boldsymbol{u}_{h}$ for $\boldsymbol{x} \in \mathrm{D}$ for every $\mathrm{D} \in \mathfrak{M}_{h}^{\mathcal{D}}$, and the discrete scalar field $p_{h} \in \mathfrak{D}_{h}$ with the corresponding piecewise constant scalar field $p_{h}(\boldsymbol{x})=p_{\mathrm{D}}$ for $\boldsymbol{x} \in \mathrm{D}$ for every $\mathrm{D} \in \mathfrak{M}_{h}^{\mathcal{D}}$. These two piecewise constant fields on mesh partition $\mathfrak{M}_{h}^{\mathcal{D}}$ are the DDFV approximations to $\nabla \boldsymbol{u}$ and $p$, respectively. On its turn, the DDFV approximation to $\boldsymbol{u}$, the velocity field solving problem (1)-(3), is given by the triplet of piecewise constant functions still denoted by $\boldsymbol{u}_{h}$

$$
\begin{aligned}
\boldsymbol{u}_{h}(\boldsymbol{x}) & =\left(\boldsymbol{u}_{h}^{\mathcal{P}}(\boldsymbol{x}), \boldsymbol{u}_{h}^{\mathcal{V}}(\boldsymbol{x}), \boldsymbol{u}_{h}^{\mathcal{E F}}(\boldsymbol{x})\right) \\
& =\left(\sum_{\mathrm{p} \in \mathcal{P}} \boldsymbol{u}_{\mathrm{p}} \chi_{\mathrm{p}}(\boldsymbol{x}), \sum_{\mathrm{v} \in \mathcal{V}} \boldsymbol{u}_{\mathrm{v}} \chi_{\mathrm{v}}(\boldsymbol{x}), \sum_{\mathbf{s} \in \mathcal{E} \cup \mathcal{F}} \boldsymbol{u}_{\mathrm{s}} \chi_{\mathrm{s}}(\boldsymbol{x})\right) \quad \text { for every } \boldsymbol{x} \in \Omega,
\end{aligned}
$$


where $\chi_{\sigma}(\boldsymbol{x})$ for $\sigma \in\{\mathrm{p}, \mathrm{v}, \mathrm{s}\}$ denote the characteristic function of subset $\sigma \in \mathfrak{R}^{3}$, i.e., $\chi(\boldsymbol{x})=1$ when $\boldsymbol{x} \in \sigma, \chi(\boldsymbol{x})=0$ otherwise. For these approximations, there hold the following a priori estimates.

Theorem 3 Let $(\boldsymbol{u}, p) \in\left(H^{2}(\Omega)\right)^{3} \times H^{1}(\Omega)$ be the velocity and pressure solution fields of the steady Stokes problem (1)-(3) under hypothesis $\left(H_{1}\right)-\left(H_{3}\right)$, and such that $p$ satisfies the compatibility condition (6). Let $\left(\boldsymbol{u}_{h}, p_{h}\right) \in \mathfrak{T}_{h, 0}^{3} \times \mathfrak{D}_{h}$ be the DDFV approximations to velocity and pressure that solve the scheme's equations (39)-(41) under Assumptions $\left(A_{1}\right)-\left(A_{2}\right)$.

Then, there exists a positive constant $C_{17}$, which is independent of $h$, such that

$$
\begin{array}{r}
\left\|\boldsymbol{u}-\boldsymbol{u}_{h}\right\|_{L^{2}(\Omega)}+\left\|\nabla \boldsymbol{u}-\nabla_{h} \boldsymbol{u}_{h}\right\|_{L^{2}(\Omega)} \leq C_{17} h\left(\|\boldsymbol{u}\|_{H^{2}(\Omega)}+\|p\|_{H^{1}(\Omega)}\right), \\
\left\|p-p_{h}\right\|_{L^{2}(\Omega)} \leq C_{17} h\left(\|\boldsymbol{u}\|_{H^{2}(\Omega)}+\|p\|_{H^{1}(\Omega)}\right) .
\end{array}
$$

Proof. To prove the first theorem's inequality, we add and subtract $\boldsymbol{u}^{J}$ and $\nabla_{h} \boldsymbol{u}^{J}$ to its left-hand side, $\boldsymbol{u}^{J}$ being the center-valued interpolation of $\boldsymbol{u}$ provided by (34)-(36). Then, we apply inequality (70) from Lemma 6 , and note that $\left\|\boldsymbol{u}^{J}-\boldsymbol{u}_{h}\right\|_{L^{2}(\Omega)}=\|\| \boldsymbol{u}^{J}-\boldsymbol{u}_{h}\|\|_{\mathfrak{T}_{h}}$ and $\left\|\nabla_{h}\left(\boldsymbol{u}^{J}-\boldsymbol{u}_{h}\right)\right\|_{L^{2}(\Omega)}=\| \nabla_{h}\left(\boldsymbol{u}^{J}-\right.$ $\left.\boldsymbol{u}_{h}\right) \|_{\mathfrak{D}_{h}}$. Therefore, we have that

$$
\begin{aligned}
\| \boldsymbol{u}- & \boldsymbol{u}_{h}\left\|_{L^{2}(\Omega)}+\right\| \nabla \boldsymbol{u}-\nabla_{h} \boldsymbol{u}_{h} \|_{L^{2}(\Omega)} \\
& \leq\left\|\boldsymbol{u}-\boldsymbol{u}^{J}\right\|_{L^{2}(\Omega)}+\left\|\nabla \boldsymbol{u}-\nabla_{h} \boldsymbol{u}^{J}\right\|_{L^{2}(\Omega)}+\left\|\boldsymbol{u}^{J}-\boldsymbol{u}_{h}\right\|_{L^{2}(\Omega)}+\left\|\nabla_{h}\left(\boldsymbol{u}^{J}-\boldsymbol{u}_{h}\right)\right\|_{L^{2}(\Omega)} \\
& \leq C_{2} h\|\boldsymbol{u}\|_{H^{2}(\Omega)}+\left\|\boldsymbol{u}^{J}-\boldsymbol{u}_{h}\right\|\left\|_{\mathfrak{T}_{h}}+\right\| \nabla_{h}\left(\boldsymbol{u}^{J}-\boldsymbol{u}_{h}\right) \|_{\mathfrak{D}_{h}} .
\end{aligned}
$$

Thanks to the Poincaré inequality (69), cf. Lemma 5 , we get

$$
\left\|\boldsymbol{u}^{J}-\boldsymbol{u}_{h}\right\|_{\mathfrak{T}_{h}} \leq C_{1}|| \mid \nabla_{h}\left(\boldsymbol{u}^{J}-\boldsymbol{u}_{h}\right) \|_{\mathfrak{D}_{h}},
$$

and, then, we use the result of Theorem 2 .

To prove the second theorem's inequality, we add and subtract $p^{I}$, the mean-valued interpolation of $p$ built on mesh $\mathfrak{M}_{h}^{\mathcal{D}}$ and provided by (37), to its left-hand side, we use inequality (71) from Lemma 7 , and note that $\left\|p^{I}-p_{h}\right\|_{L^{2}(\Omega)}=\|\| p^{I}-p_{h} \|_{\mathfrak{D}_{h}}$. We have that:

$$
\begin{aligned}
\left\|p-p_{h}\right\|_{L^{2}(\Omega)}^{2} & \leq 2\left(\left\|p-p^{I}\right\|_{L^{2}(\Omega)}^{2}+\left\|p^{I}-p_{h}\right\|_{L^{2}(\Omega)}^{2}\right) \\
& \leq C_{3}^{2} \sum_{\mathrm{D} \in \mathfrak{M}_{h}^{D}} h_{\mathrm{D}}^{2}\|p\|_{H^{1}(\mathrm{D})}^{2}+\left\|p^{I}-p_{h}\right\|_{L^{2}(\Omega)}^{2} \\
& \leq C_{3}^{2} h^{2}\|p\|_{H^{1}(\Omega)}^{2}+\left\|p^{I}-p_{h}\right\|_{\mathfrak{D}_{h}}^{2} .
\end{aligned}
$$

Then, we apply the result of Theorem 2 to get a bound for the remaining term $\left\|\mid p^{I}-p_{h}\right\|_{\mathfrak{D}_{h}}$, and take the square root of the resulting inequality.

\section{Numerical experiments}

We consider two sequences of 3-D refined mesh sets $\left\{\left(\mathfrak{M}_{h}^{\mathcal{T}}, \mathfrak{M}_{h}^{\mathcal{D}}\right)_{h}^{i}\right\}$ for $i=1,2$ that partition the computational domain $\Omega=] 0,1[\times] 0,1[\times] 0,1\left[\right.$. In the first case, meshes $\mathfrak{M}_{h}^{\mathcal{P}, 1}$ are locally refined in one of the corner of domain $\Omega$. In the second case, each mesh $\mathfrak{M}_{h}^{\mathcal{P}, 2}$ is formed by a collection of hexahedral cells obtained by a conformal decomposition of an underlying tetrahedral mesh generated by the software program tetgen. It is worth noting that in the latter case neither a particular mesh structure nor nested refinements characterizes the mesh partitionings. The two plots on the top of 
Table 1: Parameters of mesh families used in the accuracy tests: $\mathrm{n}$ is the refinement level, $N_{\mathcal{P}}$ is the number of polyhedrons, $N_{\mathcal{F}}$ is the number of faces, $N_{\mathcal{E}}$ is the number of edges, $N_{\mathcal{V}}$ is the number of vertices, $N_{\mathcal{D}}$ is the number of diamond cells $h$ is the mesh size.

\begin{tabular}{c|ccccccc}
\hline Mesh & $\mathrm{n}$ & $N_{\mathcal{P}}$ & $N_{\mathcal{F}}$ & $N_{\mathcal{E}}$ & $N_{\mathcal{V}}$ & $N_{\mathcal{D}}$ & $h$ \\
\hline & 0 & 120 & 444 & 546 & 223 & 945 & $2.50010^{-1}$ \\
$\mathfrak{M}_{h}^{\mathcal{P}, 1}$ & 1 & 960 & 3216 & 3588 & 1333 & 5811 & $1.25010^{-1}$ \\
& 2 & 7680 & 24384 & 25800 & 9097 & 40275 & $6.25010^{-2}$ \\
& 3 & 32768 & 101376 & 104544 & 35937 & 298581 & $3.12510^{-2}$ \\
\hline & 0 & 176 & 600 & 698 & 275 & 2400 & $5.00010^{-1}$ \\
$\mathfrak{M}_{h}^{\mathcal{P}, 2}$ & 1 & 888 & 2865 & 3153 & 1177 & 11460 & $2.70610^{-1}$ \\
& 2 & 11444 & 35451 & 37495 & 13489 & 141804 & $1.27710^{-1}$ \\
& 3 & 61440 & 189696 & 195216 & 66961 & 1150428 & $6.48710^{-2}$ \\
\hline
\end{tabular}

Fig. 3 show the polyhedral sets $\mathcal{P}_{1}$ and $\mathcal{P}_{2}$ of $\mathfrak{M}_{h}^{\mathcal{P}, i}, i=1,2$, while in Table 1 we report the information about the size of the meshes used in our calculations. On these sequences of refined meshes, we solve the steady Stokes problem given by (1)-(3) with viscosity function

$$
\eta(x, y, z)=1+x^{2}+y^{2}+z^{2} \quad \forall(x, y, z) \in \Omega .
$$

The boundary conditions, which are explicitly introduced into the scheme by directly setting the boundary degrees of freedom of $\boldsymbol{u}_{h}$, and the source term $\boldsymbol{f}$ are considered in accordance with the exact solution fields:

$$
\begin{aligned}
& \boldsymbol{u}(x, y, z)=\left(\begin{array}{l}
\alpha_{1} \sin (2 \pi x) \cos (2 \pi y) \cos (2 \pi z) \\
\alpha_{2} \cos (2 \pi x) \sin (2 \pi y) \cos (2 \pi z) \\
\alpha_{3} \cos (2 \pi x) \cos (2 \pi y) \sin (2 \pi z)
\end{array}\right) \text { with } \alpha_{1}+\alpha_{2}+\alpha_{3}=0, \\
& p(x, y, z)=\sin (2 \pi x) \sin (2 \pi y) \sin (2 \pi z) .
\end{aligned}
$$

The relative approximation errors are then defined for the exact solution fields $\boldsymbol{u}, \nabla \boldsymbol{u}$ and $p$ by:

$$
\begin{aligned}
\operatorname{Error}(\boldsymbol{u}) & =\frac{\left\|\boldsymbol{u}^{J}-\boldsymbol{u}_{h} \mid\right\|_{\mathfrak{T}_{h}}}{\|\| \boldsymbol{u}^{J} \|_{\mathfrak{T}_{h}}}, \\
\operatorname{Error}(\nabla \boldsymbol{u}) & =\frac{\left\|\mid \nabla_{h}\left(\boldsymbol{u}^{J}-\boldsymbol{u}_{h}\right)\right\| \|_{\mathfrak{D}_{h}}}{\left.\left\||| \nabla_{h}\left(\boldsymbol{u}^{J}\right)\right\|\right|_{\mathfrak{D}_{h}}}, \\
\operatorname{Error}(p) & =\frac{\left\|\mid p^{I}-p_{h}\right\|_{\mathfrak{D}_{h}}}{\left\|p^{I}\right\|_{\mathfrak{D}_{h}}},
\end{aligned}
$$

where in (148) we use norm (55), in (149) we use norm (51), and in (150) we use norm (49).

Fig. 3 shows the relative approximation errors defined in (148)-(150) for the numerical approximations of $\boldsymbol{u}, \nabla \boldsymbol{u}$, and $p$ using the stabilization parameter $\lambda=10^{-3}$ and the mesh families $\left(\mathfrak{M}_{h}^{\mathcal{T}}, \mathfrak{M}_{h}^{\mathcal{D}}\right)^{1}$ (left plot) and $\left(\mathfrak{M}_{h}^{\mathcal{T}}, \mathfrak{M}_{h}^{\mathcal{D}}\right)^{2}$ (right plot). The good convergence behavior of the scheme is reflected by the slopes of the experimental error curves, which are to be compared with the theoretical $\mathcal{O}(h)$ and $\mathcal{O}\left(h^{2}\right)$ slopes reported in the bottom-left corner of each plot. In particular, a second-order convergence rate seems to characterize the error curves in the left plot, i.e., when the calculation is run using the locally refined meshes of $\left(\mathfrak{M}_{h}^{\mathcal{T}}, \mathfrak{M}_{h}^{\mathcal{D}}\right)^{1}$. The convergence rate shown by the numerical results in the right plot also seems better than one, the theoretical order predicted by Theorem 2. This fact allows us to conjecture that the estimate provided by Theorem 2 might not be optimal, and that a superconvergence effect could influence the observed numerical rates. We point it out that this situation is rather 
typical of many families of finite volume methods also including the DDFV method and that, for such schemes, the theoretical proof of a second-order convergence rate under very general condition is still an open issue. Regarding the velocity gradient, the plots in Fig. 3 display a linear convergence rate, which is perfectly in agreement with the theoretical prediction. Instead, in both plots the numerical pressure begins to converge from the second mesh, and seems too converge at a faster rate from the second to the third mesh and eventually to stabilize to the expected theoretical rate.

\section{Conclusions}

In this work, we developed and analysed a DDFV method for the numerical approximation of the 3-D Stokes problem with variable viscosity coefficient. This method can be applied to general polyhedral meshes, possibly with non-conforming and non-convex elements. Since the mesh definition is a key point of all the DDFV formulations, before giving the scheme formulation we discussed the assumptions on the mesh and its construction thoroughly. Theoretical analysis allowed us to prove the uniform stability and well-posedness of such a discretization under quite general assumptions. We also proved the convergence of the velocity variable, its gradient and the pressure field, and derived a priori estimates for the approximation errors. Numerical experiments essentially confirm the theoretical predictions.

\section{References}

[1] B. Andreianov, F. Boyer, and F. Hubert. Discrete duality finite volume schemes for Leray-Lions type elliptic problems on general 2D-meshes. Num. Meth. for PDEs, 23(1):145-195, 2007.

[2] B. Andreianov, M. Gutnic, and P. Wittbold. Convergence of finite volume approximations for a nonlinear elliptic-parabolic problem: a "continuous" approach. SIAM J. Numer. Anal., 42(1):228$251,2004$.

[3] L. Beirão da Veiga. A residual based error estimator for the mimetic finite difference method. Numer. Math., 108(3):387-406, 2008.

[4] L. Beirão da Veiga, J. Droniou, and G. Manzini. A unified approach to handle convection terms in finite volumes and mimetic discretization methods for elliptic problems. Technical Report IMATI Tech. Report 23PV09/19/0, IMATI-CNR, 2009. Submitted to IMA J. Numer. Anal.

[5] L. Beirão da Veiga, V. Gyrya, K. Lipnikov, and G. Manzini. Mimetic finite difference method for the Stokes problem on polygonal meshes. J. Comput. Phys., 228(19):7215-7232, 2009.

[6] L. Beirão da Veiga, K. Lipnikov, and G. Manzini. Convergence analysis of the high-order mimetic finite difference method. Numer. Math., 113(3):325-356, 2009.

[7] L. Beirão da Veiga, K. Lipnikov, and G. Manzini. Error analysis for a mimetic discretization method of the steady Stokes problem on polyhedral meshes. SIAM, J. Numer. Anal., 48(4):1419$1443,2010$.

[8] L. Beirão da Veiga and G. Manzini. An a posteriori error estimator for the mimetic finite difference approximation of elliptic problems. Int. J. for Numer. Meth. in Engnr., 76(11):1696-1723, 2008.

[9] L. Beirão da Veiga and G. Manzini. A higher-order formulation of the mimetic finite difference method. SIAM J. Sci. Comput., 31(1):732-760, 2008.

[10] E. Bertolazzi and G. Manzini. On vertex reconstructions for cell-centered finite volume approximations of 2-D anisotropic diffusion problems. Math. Models Methods Appl. Sci., 17(1):1-32, 2007. 
[11] P. Bochev and M. D. Gunzburger. Least-Squares Finite Element Methods, volume 166 of Applied Mathematical Sciences. Springer, New York, Berlin, March 2009.

[12] F. Boyer and F. Hubert. Finite volume method for 2D linear and nonlinear elliptic problems with discontinuities. SIAM J. Numer. Anal., 46(6):3032-3070, 2008.

[13] F. Boyer, F. Hubert, and S. Krell. Non-overlapping Schwarz algorithm for solving 2D m-DDFV schemes. IMA J. N. A., 30(4):1062-1100, 2010.

[14] F. Brezzi and M. Fortin. Mixed and hybrid finite element methods, volume 15 of Springer Series in Computational Mathematics. Springer-Verlag, New York, 1991.

[15] F. Brezzi and J. Pitkäranta. On the stabilization of finite element approximations of the Stokes equations. In Efficient solutions of elliptic systems (Kiel, 1984), volume 10 of Notes Numer. Fluid Mech., pages 11-19. Vieweg, Braunschweig, 1984.

[16] A. Cangiani and G. Manzini. Flux reconstruction and pressure post-processing in mimetic finite difference methods. Comput. Methods Appl. Mech. Engrg., 197/9-12:933-945, 2008.

[17] A. Cangiani, G. Manzini, and A. Russo. Convergence analysis of a mimetic finite difference method for general second-order elliptic problems. SIAM J. Numer. Anal., 47(4):2612-2637, 2009.

[18] Y. Coudière and F. Hubert. A 3D discrete duality finite volume method for nonlinear elliptic equations. SIAM, J. Sci. Comput. (to appear).

[19] Y. Coudière, C. Pierre, and R. Turpault. A DDFV scheme for anisotropic and heterogeneous elliptic equations, application to a bio-mathematics problem: Electrocardiogram simulation. In R. Eymard and J. M. Herard, editors, Proceedings of Finite Volumes for Complex Applications V. Wiley, 2008.

[20] Y. Coudière, J.-P. Vila, and P. Villedieu. Convergence rate of a finite volume scheme for a twodimensional convection-diffusion problem. M2AN Math. Model. Numer. Anal., 33(3):493-516, 1999.

[21] Yves Coudière and Gianmarco Manzini. The discrete duality finite volume method for convectiondiffusion problems. SIAM Journal on Numerical Analysis, 47(6):4163-4192, 2010.

[22] S. Delcourte. Développement de méthodes de volumes finis pour la mécanique des fluides. $\mathrm{PhD}$ thesis, available online at http://tel.archives-ouvertes.fr/tel-00200833/fr/, 2007.

[23] S. Delcourte, K. Domelevo, and P. Omnes. A discrete duality finite volume approach to Hodge decomposition and div-curl problems on almost arbitrary two-dimensional meshes. SIAM J. Numer. Anal., 45(3):1142-1174, 2007.

[24] K. Domelevo and P. Omnes. A finite volume method for the Laplace equation on almost arbitrary two-dimensional grids. M2AN Math. Model. Numer. Anal., 39(6):1203-1249, 2005.

[25] J. Droniou and R. Eymard. A mixed finite volume scheme for anisotropic diffusion problems on any grid. Numer. Math., 105(1):35-71, 2006.

[26] J. Droniou and R. Eymard. Study of the mixed finite volume method for Stokes and Navier-Stokes equations. Numerical Methods for Partial Differential Equations, 25(1):137-171, 2009.

[27] R. Eymard, T. Gallouët, and R. Herbin. The finite volume method. In P. Ciarlet and J.L. Lions, editors, Handbook for Numerical Analysis, pages 715-1022. North Holland, 2000.

[28] R. Eymard, T. Gallouët, and R. Herbin. A cell-centered finite-volume approximation for anisotropic diffusion operators on unstructured meshes in any space dimension. IMA J. Numer. Anal., 26(2):326-353, 2006. 
[29] R. Eymard, G. Henry, R. Herbin, F. Hubert, R. Kloefkorn, and Manzini G. 3D benchmark on discretization schemes for anisotropic diffusion problems on general grid. In J. Fort, J. Furst, J. Halama, R. Herbin, and F. Hubert, editors, Proceedings of Finite Volumes for Complex Applications VI. Springer, 2011.

[30] V. Girault and P.-A. Raviart. Finite element methods for Navier-Stokes equations, volume 5 of Springer Series in Computational Mathematics. Springer-Verlag, Berlin, 1986. Theory and algorithms.

[31] F. Hermeline. A finite volume method for the approximation of diffusion operators on distorted meshes. J. Comput. Phys., 160(2):481-499, 2000.

[32] F. Hermeline. Approximation of 2-D and 3-D diffusion operators with variable full tensor coefficients on arbitrary meshes. Comput. Methods Appl. Mech. Engrg., 196(21-24):2497-2526, 2007.

[33] S. Krell. Stabilized DDFV scheme for Stokes problem with variable viscosity on general 2D meshes. Published online in Numer. Methods PDE, 2010.

[34] G. Manzini and M. Putti. Mesh locking effects in the finite volume solution of 2-D anisotropic diffusion equations. J. Comput. Phys., 220(2):751-771, 2007.

[35] G. Manzini and A. Russo. A finite volume method for advection-diffusion problems in convectiondominated regimes. Comput. Methods Appl. Mech. Engrg., 197(13-16):1242-1261, 2008.

\section{Appendix}

Lemma A.1 There exists a positive constant $C_{18}$ such that for any bounded polyhedral set $\mathcal{P} \subset \mathfrak{R}^{3}$ with positive measure $m_{\mathcal{P}}$, any planar surface $\sigma \in \mathfrak{R}^{2}$ and any function $v \in H^{1}\left(\mathfrak{R}^{3}\right)$, we have that

$$
\left|\frac{1}{|\sigma|} \int_{\sigma} v d S-\frac{1}{m_{\mathcal{P}}} \int_{\mathcal{P}} v d V\right|^{2} \leq C_{18} \frac{\operatorname{diam}\left(\widehat{\mathcal{P}_{\sigma}}\right)}{|\sigma|} \int_{\widehat{\mathcal{P}}_{\sigma}}|\nabla v|^{2} d V
$$

where $\widehat{\mathcal{P}}_{\sigma}$ is the convex hull of $\mathcal{P} \cup \sigma$.

Proof. See [26].

For simplicity of notation, in the next formulas we will implicitly refer the six points A, B, E, F, K, L to each diamond cell determined by the summation index D.

Proposition A.1 Let $\mathfrak{M}_{h}^{\mathcal{T}}=\left(\mathfrak{M}_{h}^{\mathcal{P}}, \mathfrak{M}_{h}^{\mathcal{V}}, \mathfrak{M}_{h}^{\mathcal{E F}}\right)$. For every $\boldsymbol{v} \in \mathfrak{T}_{h, 0}^{3}$ the following identities are satisfied:

(i) for any $s \in \mathfrak{M}_{h}^{\mathcal{E F}}$ there holds:

$$
\sum_{D \in \mathfrak{M}_{h}^{D} \mid s} \frac{\boldsymbol{v}_{\mathrm{L}}-\boldsymbol{v}_{\mathrm{K}}}{2} \times\left(\boldsymbol{x}_{\mathrm{B}}-\boldsymbol{x}_{\mathrm{A}}\right)=0 \quad \text { and } \quad \sum_{D \in \mathfrak{M}_{h}^{\mathcal{D}} \mid s} \frac{\boldsymbol{v}_{\mathrm{B}}-\boldsymbol{v}_{\mathrm{A}}}{2} \times\left(\boldsymbol{x}_{\mathrm{L}}-\boldsymbol{x}_{\mathrm{K}}\right)=0 ;
$$

(ii) for any $p \in \mathfrak{M}_{h}^{\mathcal{P}}$ there holds:

$$
\sum_{D \in \mathfrak{M}_{h \mid p}^{D}} \frac{\boldsymbol{v}_{\mathrm{A}}-\boldsymbol{v}_{\mathrm{B}}}{2} \times\left(\boldsymbol{x}_{\mathrm{F}}-\boldsymbol{x}_{\mathrm{E}}\right)=0 \quad \text { and } \quad \sum_{D \in \mathfrak{M}_{h \mid p}^{D}} \frac{\boldsymbol{v}_{\mathrm{F}}-\boldsymbol{v}_{\mathrm{E}}}{2} \times\left(\boldsymbol{x}_{\mathrm{B}}-\boldsymbol{x}_{\mathrm{A}}\right)=0 ;
$$


(iii) for any $v \in \mathfrak{M}_{h}^{\mathcal{V}}$ there holds:

$$
\sum_{\left.D \in \mathfrak{M}_{h}^{D}\right|_{\mathcal{v}}} \frac{\boldsymbol{v}_{\mathrm{L}}-\boldsymbol{v}_{\mathrm{K}}}{2} \times\left(\boldsymbol{x}_{\mathrm{F}}-\boldsymbol{x}_{\mathrm{E}}\right)=0 \quad \text { and } \quad \sum_{D \in \mathfrak{M}_{\left.h\right|^{\mathcal{V}}}^{\mathcal{D}}} \frac{\boldsymbol{v}_{\mathrm{E}}-\boldsymbol{v}_{\mathrm{F}}}{2} \times\left(x_{\mathrm{L}}-\boldsymbol{x}_{\mathrm{K}}\right)=0 .
$$

Proof. Let $\mathrm{D}=\mathrm{D}_{(\mathrm{e}, \mathrm{f})}$ be the diamond cell of $\mathfrak{M}_{h}^{\mathcal{D}}$ uniquely determined by the admissible pair $(\mathrm{e}, \mathrm{f}) \in$ $\mathcal{E} \times \mathcal{F}$.

(i). First, we consider the case $\mathbf{s}=\mathrm{f}$, and denote the point of $\mathrm{D}$ associated to $\mathrm{f}$ by $\mathrm{F}$. Note that

$$
\sum_{\mathrm{D} \in \mathfrak{M}_{h \mid \mathrm{F}}^{\mathcal{D}}}\left(\boldsymbol{x}_{\mathrm{B}}-\boldsymbol{x}_{\mathrm{A}}\right)=0
$$

because the sequence of face edges $\mathrm{e}=\mathrm{v}_{\mathrm{B}}-\mathrm{v}_{\mathrm{A}}=\overline{\boldsymbol{x}_{\mathrm{A}} \boldsymbol{x}_{\mathrm{B}}}$ form a closed loop, i.e., a telescopic sum whose first and last terms are coincident. The first relation of item $(i)$ follows immediately by using (A.2) in:

$$
\sum_{\mathrm{D} \in \mathfrak{M}_{h}^{\mathcal{D}}} \frac{\boldsymbol{v}_{\mathrm{L}}-\boldsymbol{v}_{\mathrm{K}}}{2} \times\left(\boldsymbol{x}_{\mathrm{B}}-\boldsymbol{x}_{\mathrm{A}}\right)=\frac{\boldsymbol{v}_{\mathrm{L}}-\boldsymbol{v}_{\mathrm{K}}}{2} \times \sum_{\mathrm{D} \in \mathfrak{M}_{h \mid \mathrm{F}}^{\mathcal{D}}}\left(\boldsymbol{x}_{\mathrm{B}}-\boldsymbol{x}_{\mathrm{A}}\right)=0
$$

The second relation follows by using the same arguments after exchanging the role of $\boldsymbol{x}_{\mathrm{A}}, \boldsymbol{x}_{\mathrm{B}}$ and $\boldsymbol{v}_{\mathrm{A}}$, $\boldsymbol{v}_{\mathrm{B}}$.

Then, we consider the case $\mathrm{s}=\mathrm{e}$, and denote the point associated to edge e by E. Note that

$$
\sum_{\mathrm{D} \in \mathfrak{M}_{h}^{\mathcal{D}} \mid \mathrm{E}} \frac{\boldsymbol{v}_{\mathrm{L}}-\boldsymbol{v}_{\mathrm{K}}}{2}=0
$$

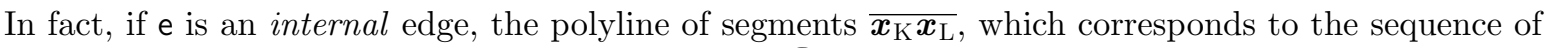
primal cells $\mathrm{p}_{\mathrm{K}} \rightarrow \mathrm{p}_{\mathrm{L}}$ around the edge e for $\mathrm{D} \in \mathfrak{M}_{h \mid \mathrm{E}}^{\mathcal{D}}$, forms a closed loop, as in the previous case. On the other hand, if e is a boundary edge, it must belong to two distinct boundary faces. Therefore, we can reorder the summation to begin from one of the boundary faces and end up to the other one, and the telescopic sum (A.3) equals the difference of the terms $\boldsymbol{v}_{\mathrm{L}}$ of these two faces. Now, we recall that $\mathrm{L}$ coincides with $\mathrm{F}$ if $\mathrm{f}$ is a boundary face, and identity (A.3) is true because the hypothesis that $\boldsymbol{v} \in \mathfrak{T}_{h, 0}^{3}$ implies that $\boldsymbol{v}_{\mathrm{L}}=\boldsymbol{v}_{\mathrm{F}}=0$. The first relation of item (i) follows by using (A.3) in

$$
\left(\sum_{D \in \mathfrak{M}_{h \mid \mathrm{E}}^{D}} \frac{\boldsymbol{v}_{\mathrm{L}}-\boldsymbol{v}_{\mathrm{K}}}{2}\right) \times\left(\boldsymbol{x}_{\mathrm{B}}-\boldsymbol{x}_{\mathrm{A}}\right)=0
$$

If $\mathrm{e}$ is an internal edge the second relation follows by using the same argument after exchanging the role of $\boldsymbol{x}_{\mathrm{A}}, \boldsymbol{x}_{\mathrm{B}}$ and $\boldsymbol{v}_{\mathrm{A}}, \boldsymbol{v}_{\mathrm{B}}$. If e is a boundary edge, the second relation is true since both $\mathrm{v}_{\mathrm{A}}$ and $\mathrm{v}_{\mathrm{B}}$, i.e., A and B, are on the boundary of $\Omega$, and $\boldsymbol{v} \in \mathfrak{T}_{h, 0}^{3}$ implies again that $\boldsymbol{v}_{\mathrm{A}}=\boldsymbol{v}_{\mathrm{B}}=0$.

(ii). The left-hand side of the first relation of item (ii) can be split as

$$
\begin{aligned}
\sum_{\left.\mathrm{D} \in \mathfrak{M}_{h}^{\mathcal{D}}\right|_{\mathrm{p}}} \frac{\boldsymbol{v}_{\mathrm{A}}-\boldsymbol{v}_{\mathrm{B}}}{2} \times\left(\boldsymbol{x}_{\mathrm{F}}-\boldsymbol{x}_{\mathrm{E}}\right)= & \sum_{\mathrm{f} \in \partial \mathrm{p}}\left(\sum_{\left.\mathrm{D} \in \mathfrak{M}_{h}^{\mathcal{D}}\right|_{\mathrm{F}}} \frac{\boldsymbol{v}_{\mathrm{A}}-\boldsymbol{v}_{\mathrm{B}}}{2}\right) \times \boldsymbol{x}_{\mathrm{F}} \\
& -\sum_{\mathrm{e} \in \partial \mathrm{p}}\left(\sum_{\left.\mathrm{D} \in \mathfrak{M}_{h}^{D}{ }_{\mid \mathrm{E}} \cap \mathfrak{M}_{h}^{\mathcal{D}}\right|_{\mathrm{p}}} \frac{\boldsymbol{v}_{\mathrm{A}}-\boldsymbol{v}_{\mathrm{B}}}{2}\right) \times \boldsymbol{x}_{\mathrm{E}} .
\end{aligned}
$$

Thanks to (A.2), the first term of the right-hand side of (A.4) is zero. 
Then, we also note that for any edge e that belongs to $\mathrm{p}$ there holds:

$$
\sum_{\mathrm{D} \in \mathfrak{M}_{h \mid \mathrm{E}}^{D} \cap \mathfrak{M}_{h \mid \mathrm{p}}^{D}} \frac{\boldsymbol{v}_{\mathrm{A}}-\boldsymbol{v}_{\mathrm{B}}}{2}=0 .
$$

In fact, for any edge e there exists two and only two faces in $\mathrm{p}$ to which e belongs, and thus only two distinct diamonds $D_{(e, f)}$. Consistently with the face and edge orientation, the segment corresponding to edge e, which connects $\boldsymbol{x}_{\mathrm{A}}$ and $\boldsymbol{x}_{\mathrm{B}}$, is differently oriented in these two diamonds. The second relation follows by using the same argument after exchanging the role of $\boldsymbol{v}_{\mathrm{E}}, \boldsymbol{v}_{\mathrm{F}}$ with $\boldsymbol{x}_{\mathrm{E}}, \boldsymbol{x}_{\mathrm{F}}$.

(iii). Let us note that the left-hand side of the first relation of item (iii) can be split as

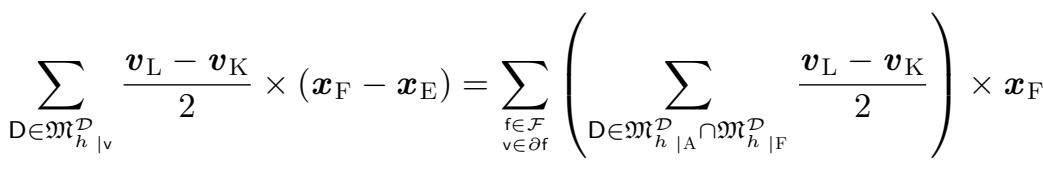

$$
\begin{aligned}
& -\sum_{\substack{\in \in \mathcal{E} \\
v \in \mathcal{e}}}\left(\sum_{\substack{D \in M_{h}^{D} \\
\mid \mathrm{E}}} \frac{\boldsymbol{v}_{\mathrm{L}}-\boldsymbol{v}_{\mathrm{K}}}{2}\right) \times \boldsymbol{x}_{\mathrm{E}} .
\end{aligned}
$$

The second term in the right-hand side of (A.6) is zero thanks to (A.3). Then we note that a vertex $v$ and a face $f$ to which this node belongs only determine two diamonds, and that face $f$ determines uniquely the primal cells $\mathrm{p}_{\mathrm{K}}$ and $\mathrm{p}_{\mathrm{L}}$. Here, we implicitly assume that $\mathrm{p}_{\mathrm{L}}$ may be a degenerate cell with zero volume for $\mathrm{L}=\mathrm{F}$ if $\mathrm{f}$ is a boundary face. Moreover, the face and the edge orientation implies that the segment connecting $\boldsymbol{x}_{\mathrm{K}}$ to $\boldsymbol{x}_{\mathrm{L}}$ in the first diamond is oriented opposite to the segment connecting the same cell centers in the second diamond. Thus, for such a pair $(v, f)$ there holds that

$$
\sum_{\left.\left.\mathrm{D} \in \mathfrak{M}_{h}^{D}\right|_{\mid \vee} \cap \mathfrak{M}_{h}^{D}\right|_{\mathrm{F}}} \frac{\boldsymbol{v}_{\mathrm{L}}-\boldsymbol{v}_{\mathrm{K}}}{2}=0,
$$

from which the final relation follows. The second relation of item (iii) follows by using the same argument after exchanging the role of $\boldsymbol{v}_{\mathrm{K}}, \boldsymbol{v}_{\mathrm{L}}$ and $\boldsymbol{x}_{\mathrm{K}}, \boldsymbol{x}_{\mathrm{L}}$. 


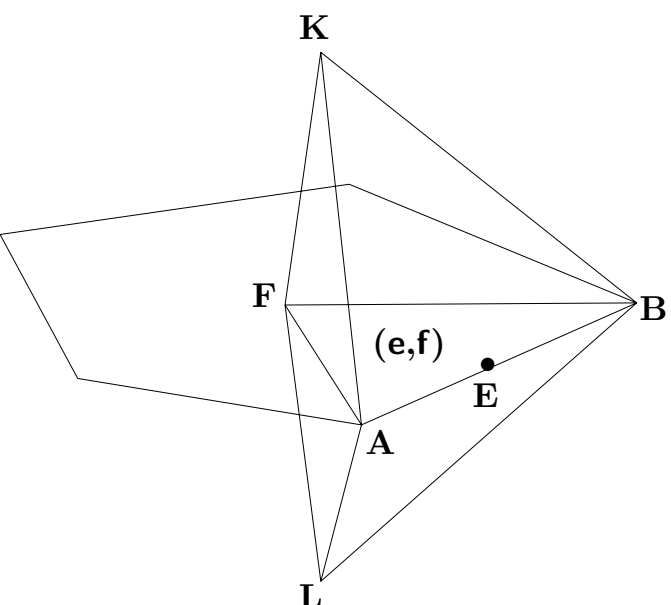

(a) Diamond cell $\mathrm{D}_{(\mathrm{e}, \mathrm{f})}$

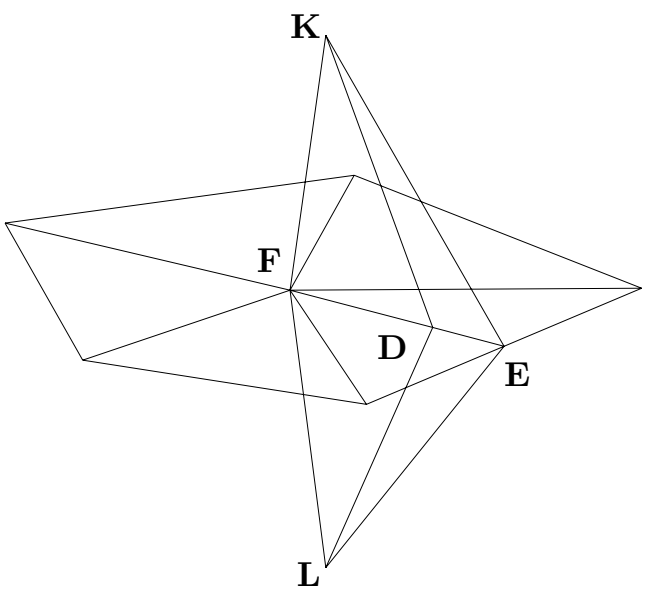

(c) The internal surface $\mathcal{S}_{\mathrm{D}, \mathrm{AB}}$

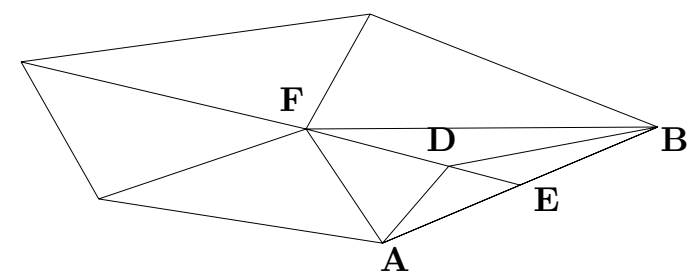

(b) The internal surface $\mathcal{S}_{\mathrm{D}, \mathrm{KL}}$

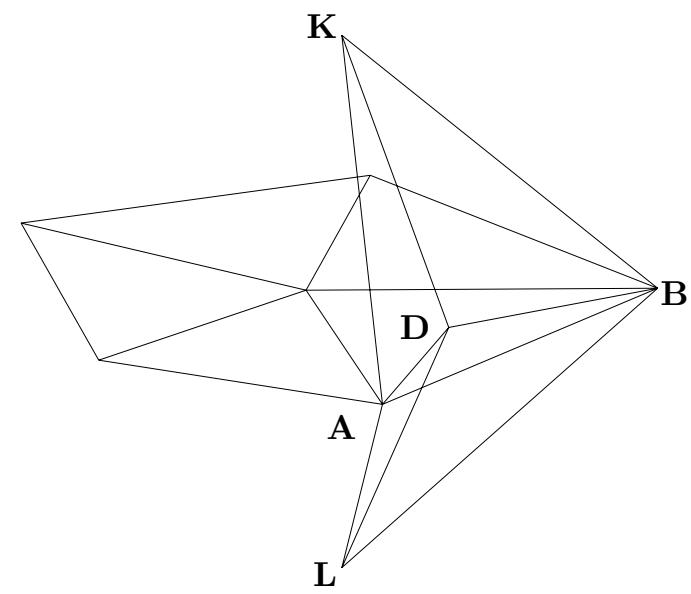

(d) The internal surface $\mathcal{S}_{\mathrm{D}, \mathrm{EF}}$

Figure 1: Construction of the diamond mesh: $(a)$, the diamond cell $\mathrm{D}_{(\mathrm{e}, \mathrm{f})} ;(b)$, the internal surface $\mathcal{S}_{\mathrm{D}, \mathrm{KL}}$ used to characterize the control volumes $\mathrm{p}_{\mathrm{K}}$ and $\mathrm{p}_{\mathrm{L}}$ of mesh $\mathfrak{M}_{h}^{\mathcal{P}} ;(c)$, the internal surface $\mathcal{S}_{\mathrm{D}, \mathrm{AB}}$ used to build the control volumes $\mathrm{v}_{\mathrm{A}}$ and $\mathrm{v}_{\mathrm{B}}$ of mesh $\mathfrak{M}_{h}^{\mathcal{V}} ;(d)$, the internal surface $\mathcal{S}_{\mathrm{D}, \mathrm{EF}}$ used to build the control volumes $\mathrm{s}_{\mathrm{E}}$ and $\mathrm{s}_{\mathrm{F}}$ of mesh $\mathfrak{M}_{h}^{\mathcal{E F}}$. 

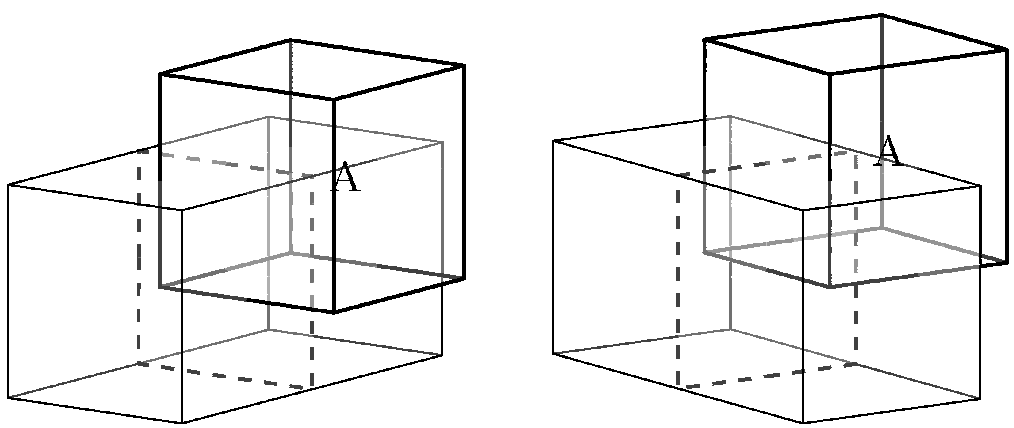

(a)
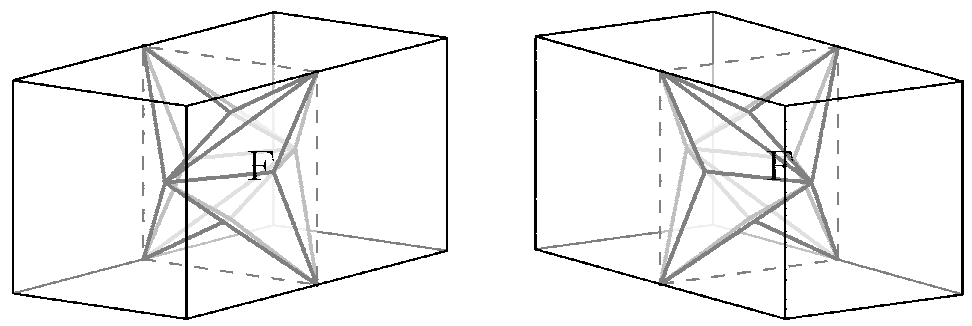

(b)
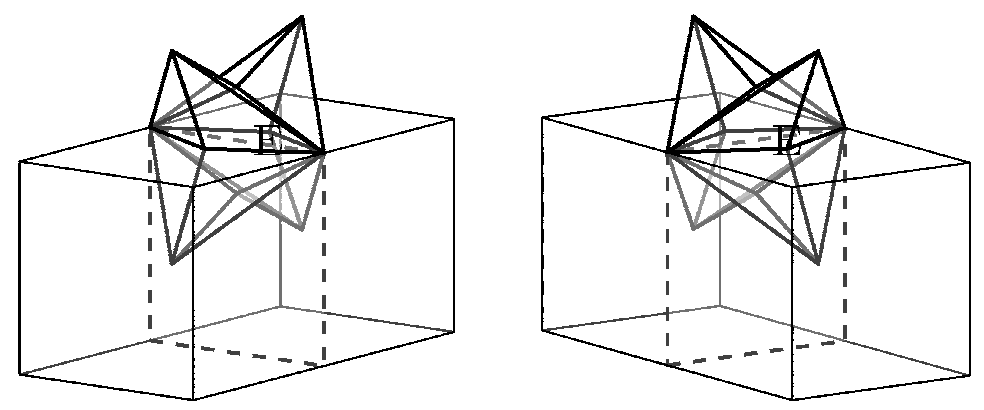

(c)

Figure 2: Construction of dual meshes $\mathfrak{M}_{h}^{\mathcal{V}}$ and $\mathfrak{M}_{h}^{\mathcal{E F}}$ from a cubic primal mesh $\mathfrak{M}_{h}^{\mathcal{P}}$. In $(a),(b)$ and (c) we show two adjacent cubic cells of $\mathfrak{M}_{h}^{\mathcal{P}}$ (thin solid lines) and one dual cell (thick solid line). The left and the rights plots show the same group of two primary cells and one dual cell from two different viewpoints. The interface separating the two primary cells in all plots is drawn using dashed lines. In plot $(a)$ we show a dual cell of type vertex, i.e., a cell that belongs to $\mathfrak{M}_{h}^{\mathcal{V}}$; in plot (b) we show a dual cell of type face and in plot $(c)$ we show a dual cell of type edge, i.e., cells that belong to $\mathfrak{M}_{h}^{\mathcal{E F}}$. 


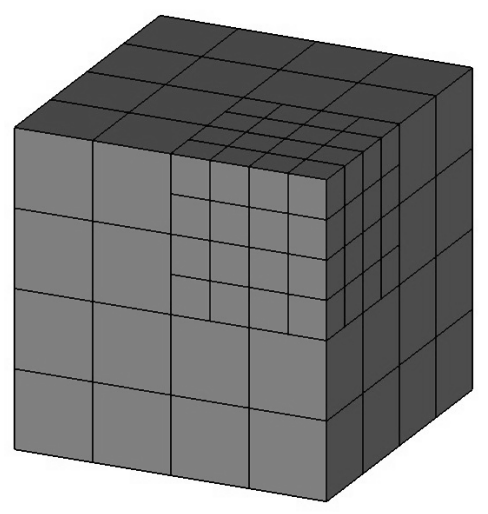

(a) Polyhedral set $\mathcal{P}_{1}$

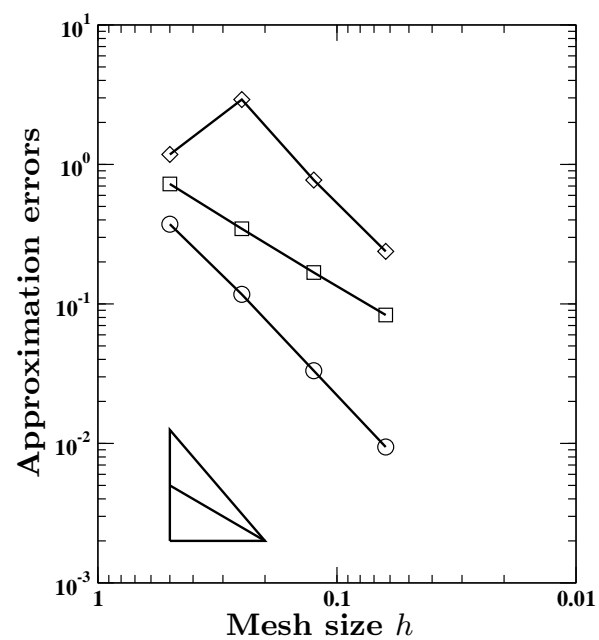

(c) Mesh $\left(\mathfrak{M}_{h}^{\mathcal{P}}, \mathfrak{M}_{h}^{\mathcal{D}}\right)^{1}$

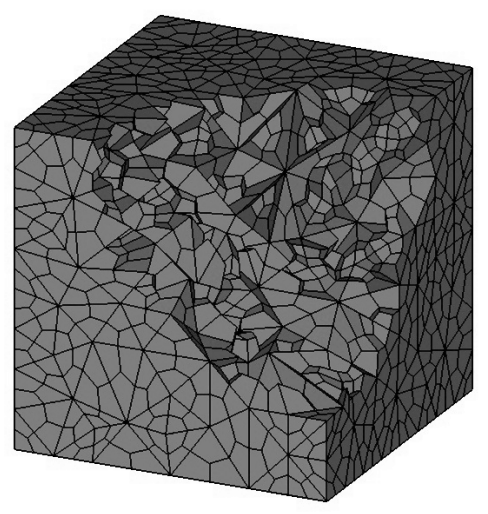

(b) Polyhedral set $\mathcal{P}_{2}$

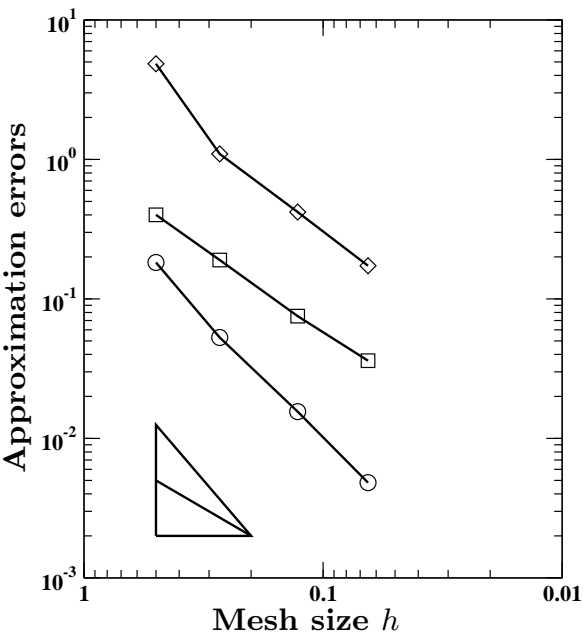

(d) Mesh $\left(\mathfrak{M}_{h}^{\mathcal{P}}, \mathfrak{M}_{h}^{\mathcal{D}}\right)^{2}$

Figure 3: Accuracy test. Plots $(a)-(b)$ display the polyhedral sets $\mathcal{P}^{1}$ and $\mathcal{P}^{2}$ of the first mesh sets of the two mesh families $\left(\mathfrak{M}_{h}^{\mathcal{T}}, \mathfrak{M}_{h}^{\mathcal{D}}\right)_{h}^{1}$ and $\left(\mathfrak{M}_{h}^{\mathcal{T}}, \mathfrak{M}_{h}^{\mathcal{D}}\right)_{h}^{2}$ used in the accuracy tests. In plot $(b)$, a part of the cells around vertex $(1,1,1)$ has been removed to show the interior. The parameters of all the meshes used in the simulation are reported in Table 1. Plots $(c)-(d)$ show the approximation errors for the viscosity field $\eta$ given by (146). In each plot, we report Error $(\boldsymbol{u})$ (circles), see equation (148), $\operatorname{Error}(\nabla(\boldsymbol{u}))$ (squares), see equation (149), Error $(p)$ (diamonds), see equation (150), and two straight lines showing the theoretical slopes $\mathcal{O}(h)$ and $\mathcal{O}\left(h^{2}\right)$. 